Prepared in cooperation with the Colorado Water Conservation Board and the Colorado Department of Transportation

\title{
Regional Regression Equations for Estimation of Natural Streamflow Statistics in Colorado
}

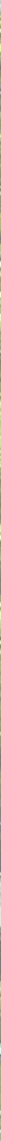

Scientific Investigations Report 2009-5136 
COVER: Arkansas River near Buena Vista, Colorado.

Photograph by Michael Lewis, U.S. Geological Survey. 


\section{Regional Regression Equations for Estimation of Natural Streamflow Statistics in Colorado}

By Joseph P. Capesius and Verlin C. Stephens

Prepared in cooperation with the Colorado Water Conservation Board and the Colorado Department of Transportation

Scientific Investigations Report 2009-5136 


\title{
U.S. Department of the Interior \\ KEN SALAZAR, Secretary
}

\author{
U.S. Geological Survey \\ Suzette M. Kimball, Acting Director
}

U.S. Geological Survey, Reston, Virginia: 2009

For more information on the USGS - the Federal source for science about the Earth, its natural and living resources, natural hazards, and the environment, visit http://www.usgs.gov or call 1-888-ASK-USGS

For an overview of USGS information products, including maps, imagery, and publications, visit http://www.usgs.gov/pubprod

To order this and other USGS information products, visit http://store.usgs.gov

Any use of trade, product, or firm names is for descriptive purposes only and does not imply endorsement by the U.S. Government.

Although this report is in the public domain, permission must be secured from the individual copyright owners to reproduce any copyrighted materials contained within this report.

Suggested citation:

Capesius, J.P., and Stephens, V.C., 2009, Regional regression equations for estimation of natural streamflow statistics in Colorado: U.S. Geological Survey Scientific Investigations Report 2009-5136, 46 p.

\section{Library of Congress Cataloging-in-Publication Data}

Capesius, Joseph P.

Regional regression equations for estimation of natural streamflow statistics in Colorado / by Joseph P. Capesius and Verlin C. Stephens

p. cm. -- (Scientific investigations report ; 2009-5136)

"Prepared in cooperation with the Colorado Water Conservation Board and the Colorado Department of Transportation."

Includes bibliographic references.

ISBN 978-1-4113-2562-3

1. Stream measurements--Colorado. 2. Streamflow--Colorado. 3. Regression analysis. I. Stephens, Verlin C. II. Geological Survey (U.S.) III. Colorado Water Conservation Board. IV. Colorado Dept. of Transportation. V. Title. VI. Series: Scientific investigations report ; 2009-5136.

GB1225.C6C36 2009

$551.48^{\prime} 309788021--d c 22$ 


\section{Contents}

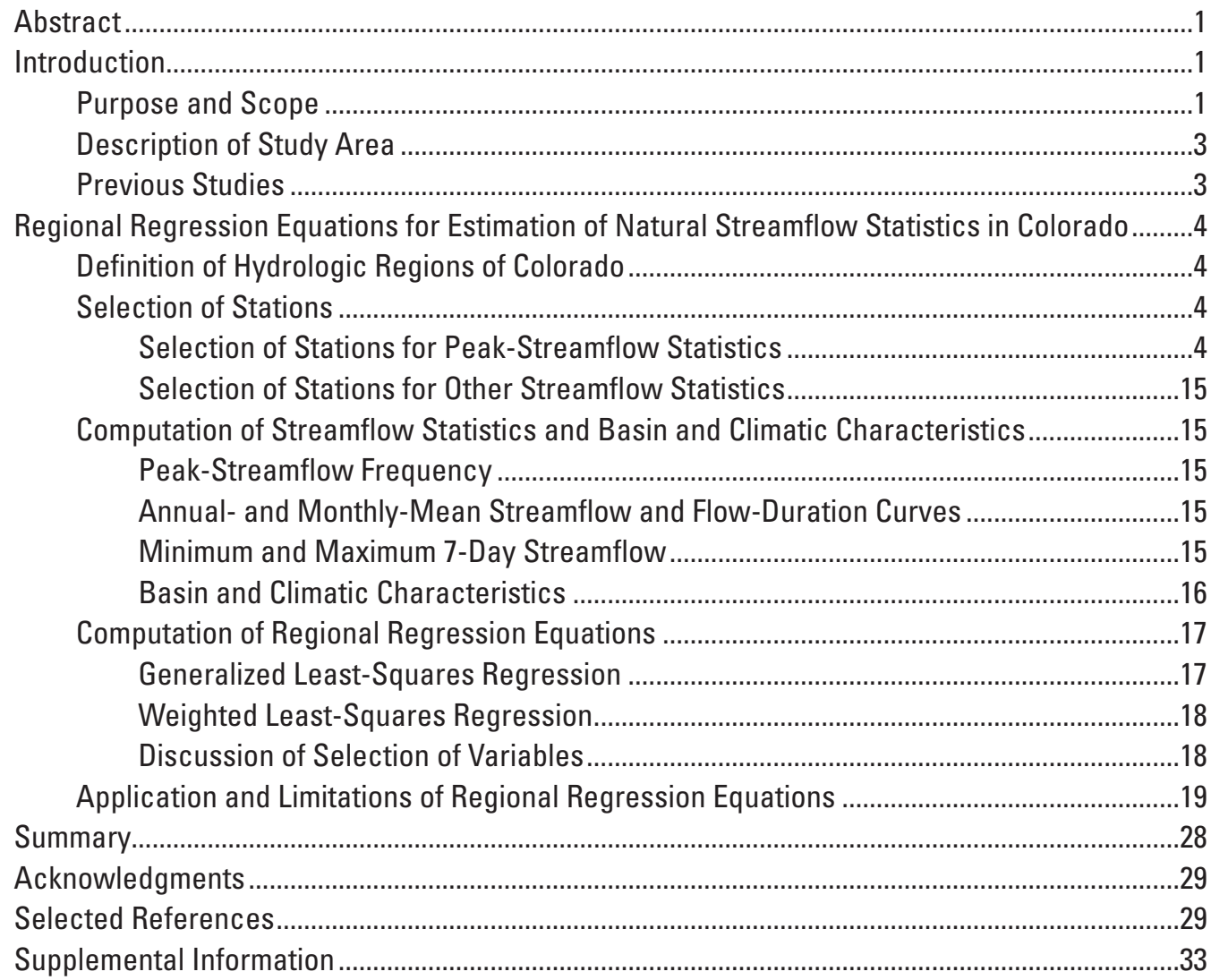

\section{Figures}

1. Boundaries of hydrologic regions and locations of streamflow gaging stations in Colorado and adjacent States.......................................................................

2. Summary of variables and regression diagnostics for regional equations ......................18

3. Regional regression equations for the Mountain hydrologic region ...............................20

4. Regional regression equations for the Northwest hydrologic region .............................22

5. Regional regression equations for the Rio Grande hydrologic region ..............................24

6. Regional regression equations for the Southwest hydrologic region .............................26

7. Regional regression equations for the Plains hydrologic region ......................................28

\section{Tables}

1. Drainage-basin and climatic characteristics at streamflow-gaging stations used for regional regression analysis...............................................................

2. Basin and climatic characteristics evaluated for use in regional

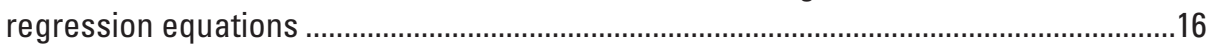

3. Sources of data used to compute basin and climatic characteristics............................17

4. Streamflow-gaging stations used in the development of the regional streamflow regression equations 


\section{Conversion Factors and Datum}

Inch/Pound to SI

\begin{tabular}{lcl}
\hline Multiply & By & To obtain \\
\hline & Length & \\
\hline inch (in.) & 2.54 & centimeter $(\mathrm{cm})$ \\
inch (in.) & 25.4 & millimeter $(\mathrm{mm})$ \\
foot (ft) & 0.3048 & meter $(\mathrm{m})$ \\
mile (mi) & 1.609 & kilometer $(\mathrm{km})$ \\
\hline & Area & \\
\hline square mile $\left(\mathrm{mi}^{2}\right)$ & 259.0 & hectare $(\mathrm{ha})$ \\
square mile $\left(\mathrm{mi}^{2}\right)$ & 2.590 & square kilometer $\left(\mathrm{km}^{2}\right)$ \\
\hline & Flow rate & \\
\hline acre-foot per day $($ acre-ft/d) & 0.01427 & cubic meter per second $\left(\mathrm{m}^{3} / \mathrm{s}\right)$ \\
acre-foot per year $($ acre-ft $/ \mathrm{yr})$ & 1,233 & cubic meter per year $\left(\mathrm{m}^{3} / \mathrm{yr}\right)$ \\
acre-foot per year $($ acre-ft/yr) & 0.001233 & cubic hectometer per year $(\mathrm{hm} 3 / \mathrm{yr})$ \\
cubic foot per second $(\mathrm{ft} / \mathrm{s})$ & 0.02832 & cubic meter per second $\left(\mathrm{m}^{3} / \mathrm{s}\right)$ \\
\hline
\end{tabular}

Vertical coordinate information is referenced to the North American Vertical Datum of 1988 (NAVD 88).

Horizontal coordinate information is referenced to the North American Datum. 


\title{
Regional Regression Equations for Estimation of Natural Streamflow Statistics in Colorado
}

\author{
By Joseph P. Capesius and Verlin C. Stephens
}

\section{Abstract}

The U.S. Geological Survey (USGS), in cooperation with the Colorado Water Conservation Board and the Colorado Department of Transportation, developed regional regression equations for estimation of various streamflow statistics that are representative of natural streamflow conditions at ungaged sites in Colorado. The equations define the statistical relations between streamflow statistics (response variables) and basin and climatic characteristics (predictor variables). The equations were developed using generalized least-squares and weighted least-squares multilinear regression reliant on logarithmic variable transformation. Streamflow statistics were derived from at least 10 years of streamflow data through about 2007 from selected USGS streamflow-gaging stations in the study area that are representative of natural-flow conditions. Basin and climatic characteristics used for equation development are drainage area, mean watershed elevation, mean watershed slope, percentage of drainage area above 7,500 feet of elevation, mean annual precipitation, and 6-hour, 100-year precipitation. For each of five hydrologic regions in Colorado, peak-streamflow equations that are based on peak-streamflow data from selected stations are presented for the 2-, 5-, 10-, 25-, 50-, 100-, 200-, and 500-year instantaneous-peak streamflows. For four of the five hydrologic regions, equations based on daily-mean streamflow data from selected stations are presented for 7-day minimum 2-, 10-, and 50-year streamflows and for 7-day maximum 2-, 10-, and 50-year streamflows. Other equations presented for the same four hydrologic regions include those for estimation of annual- and monthly-mean streamflow and streamflow-duration statistics for exceedances of 10, 25, 50, 75 , and 90 percent. All equations are reported along with salient diagnostic statistics, ranges of basin and climatic characteristics on which each equation is based, and commentary of potential bias, which is not otherwise removed by log-transformation of the variables of the equations from interpretation of residual plots. The predictor-variable ranges can be used to assess equation applicability for ungaged sites in Colorado.

\section{Introduction}

The U.S. Geological Survey (USGS), in cooperation with the Colorado Water Conservation Board and the Colorado Department of Transportation, developed regional regression equations for estimation of various streamflow statistics that are representative of natural streamflow conditions in Colorado. Accurate estimates of various streamflow statistics are important for water-resource management, stream-related structural design, stream-hazard identification, and water-quality management. Streamflow statistics routinely are needed for ungaged watersheds that lack nearby streamflow-gaging stations (stations) from which streamflow statistics could be directly computed. Regional regression equations are a common tool used to estimate streamflow statistics at ungaged sites across the Nation and Colorado.

Regional regression equations are based on statistical relations between (1) streamflow statistics of interest computed from applicable records of the stations and (2) basin and climatic characteristics, for which data are typically readily available. The use of regional equations, along with expressions of predictive uncertainty, generally represents a reliable and cost-effective means for estimating streamflow statistics at ungaged sites.

\section{Purpose and Scope}

The purpose of this report is to present regional regression equations, by hydrologic region, for estimation of streamflow statistics for naturally flowing streams in Colorado (fig. 1). The equations were developed using the statistical relations between streamflow statistics (response variables) and basin and climatic characteristics (predictor variables). The current (2009) study updates or refines the equations provided by Kircher and others (1985) and Vaill (1999) and uses streamflow data collected after those studies were completed. Each of these previous studies is described in the section "Previous Studies."

For each of five hydrologic regions in Colorado, peakstreamflow equations based on peak-streamflow data from selected stations are presented for the 2-, 5-, 10-, 25-, 50-, 100-, 200-, and 500-year instantaneous-peak streamflows (peak streamflow). For four of the five hydrologic regions, minimum and maximum 7-day streamflow (7-day, $T$-year) equations based on daily-mean streamflow data from selected stations are presented for 7-day minimum 2-, 10-, and 50-year streamflows as well as for 7-day maximum 2-, 10-, and 50-year streamflows. Other equations presented for the same four hydrologic regions include those for estimation of annual- and monthly-mean streamflow and streamflow-duration statistics for exceedances of $10,25,50,75$, and 90 percent. The streamflow-duration statistics collectively are referred to as the "flow-duration curve." The flow-duration values, presented in this report as 


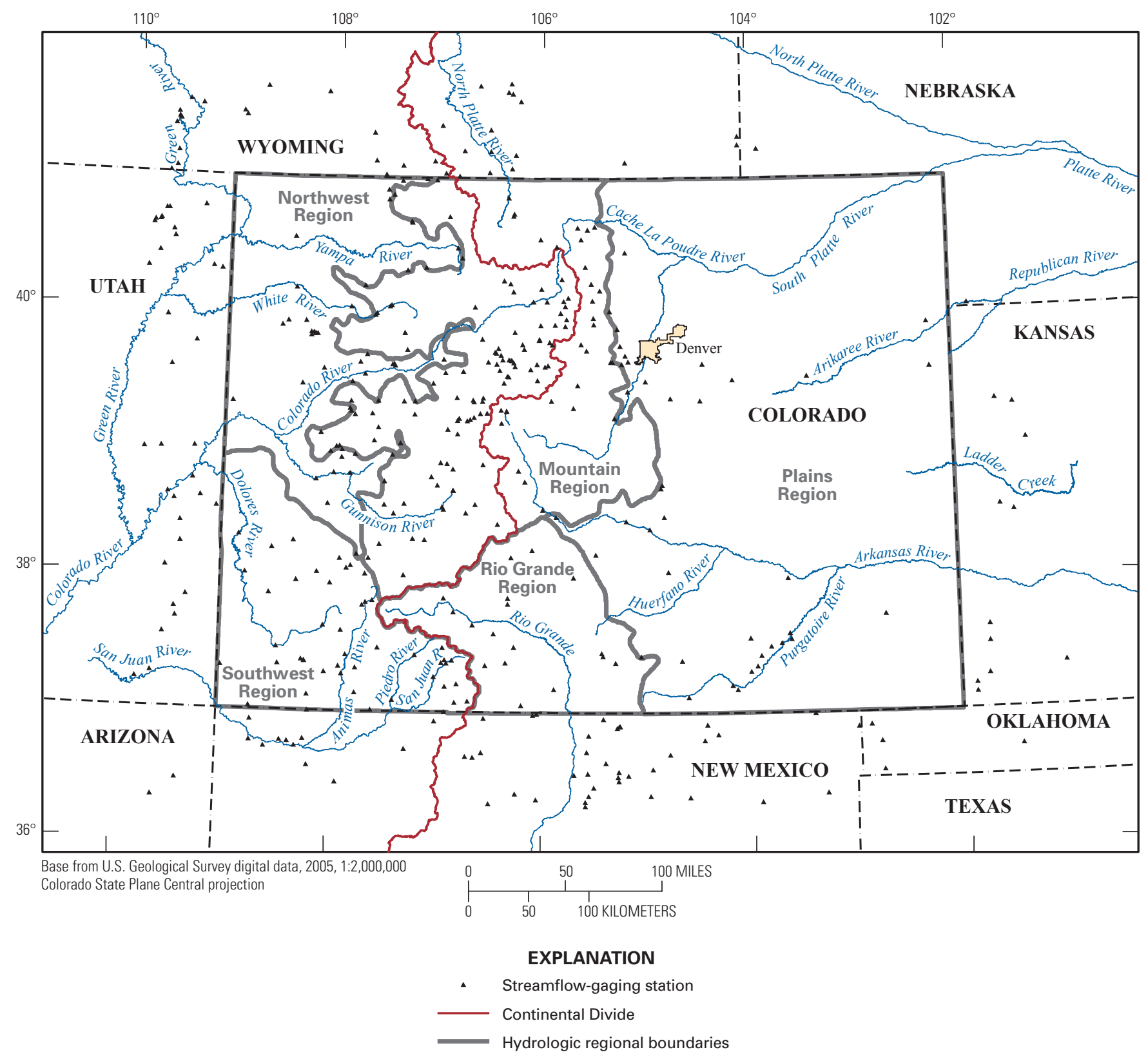

Figure 1. Boundaries of hydrologic regions and locations of streamflow gaging stations in Colorado and adjacent States.

Q10th, Q25th, Q50th, Q75th, and Q90th, indicate the percentage of days that a given daily-mean streamflow is exceeded. For example, a 10-percent flow-duration value $(Q 10 t h)$ is calculated as the daily-mean streamflow at a given station that is exceeded 10 percent of the time or 10 percent of the days. Finally, this report also describes some limitations associated with the equations, which includes an assessment of prediction errors and assessment of equation applicability for ungaged sites.

The study area of equation applicability is limited to Colorado, even though the study area was extended to include stations within a 50-mile boundary or buffer surrounding Colorado. The study area was extended 50 miles beyond the Colorado for the sole purpose of equation development.
The regional equations for peak-streamflow estimation were developed using annual peak-streamflow data collected through water year 2006 (October 1, 2005, through September 30, 2006). The remaining equations, with the exception of the 7-day, $T$-year minimum streamflow equations, were developed with data through water year 2007. The 7-day, $T$-year minimum streamflow equations were developed with data collected through climate year 2007 (April 1, 2006, through March 31, 2007). The choice of climate year in lieu of water year explicitly was made because the climate year designation splits the daily-mean streamflow record at a time (spring) in which streamflows for a given station generally are larger than at the water year (autumn) or even calendar year transition. 
The study is limited by type of statistical procedures used to define the relation between streamflow statistics and basin and climate characteristics. These procedures included generalized least-square and weighted least-square, multilinear regression based on base-10 logarithmic transformations of all included variables. The authors recognize that other transformations of variables could be used.

The study was limited to the use of several selected basin and climatic characteristics described in the section "Basin and Climatic Characteristics." These characteristics are drainage area; mean watershed slope; mean annual precipitation; mean watershed elevation; 6-hour, 100-year precipitation; and percentage of drainage area above 7,500 feet of elevation.

The equations reported here, as well as equations in all the studies referenced, are or were scoped to estimate natural streamflow statistics for ungaged sites. To clarify, the equations are based on analysis of streamflow data representing streamflow conditions relatively unaffected by anthropogenic influences such as regulation and diversion or return flows such as from a municipality or mining operation, or urban development in a basin. Kircher and others (1985) provide the most quantitative description of natural streamflow. Those authors defined natural streamflow as streamflow from drainage basins relatively unaffected by urban development or water-management activities such as substantial reservoir storage, streamflow diversions, or return flows of previously diverted streamflow. Further, those authors defined natural streamflow as streamflow having less than about 10 percent of the mean-annual streamflow volume at the station affected by anthropogenic activity. The definition by Kircher and others (1985) was used in the current (2009) study. Also, the selection of stations used for the current (2009) study was influenced by those stations selected by Kircher and others (1985), Vaill (1999), and the authors' general knowledge of hydrologic systems in Colorado.

\section{Description of Study Area}

Colorado has a varied and diverse terrain and climate. The State lies within the headwaters of the major river basins of the Colorado River, Missouri River, Rio Grande, and Arkansas River. The geographic variation in Colorado is well characterized by three major physiographic provinces, which trend north to south across the State (Fenneman, 1931).

Encompassing about 40 percent of Colorado on the east, the Great Plains Province is characterized by rolling grasslands broken by occasional hills and bluffs and shallow river valleys. Elevations in the Great Plains range from near 3,500 feet at the eastern border of Colorado to about 6,000 feet in the foothills of the Rocky Mountains at the western edge of the province. The Great Plains have low relative humidity, plentiful sunshine, limited precipitation, substantial and persistent wind, and substantial diurnal variability in air temperatures. Annual temperature extremes generally range from -10 to $-15^{\circ}$ Fahrenheit in winter to about $100^{\circ}$ Fahrenheit in summer. Most precipitation occurs as rain during April through September, largely in the form of thunderstorms. Mean annual precipitation in the Great Plains ranges from 9 to 18 inches per year (Colorado State University, 2008). Precipitation tends to decrease from the eastern border of the Great Plains to a minimum near the foothills at which point it increases rapidly with the increase in elevation of the foothills (Western Regional Climate Center, 2008).

Encompassing central Colorado from north to south, the Southern Rocky Mountains Province lies west of the Great Plains and is characterized by several mountain ranges. Elevations range from about 6,000 feet to more than 14,000 feet. The mountain ranges are characteristically separated by steep valleys and high intermontane parks. The climate of the Southern Rocky Mountains is strongly affected by differences in elevation. Wide variations occur within short distances. Similarly, temperature varies with elevation and decreases with increased elevation (Colorado Geological Survey, 2003; Colorado State University, 2008; Western Regional Climate Center, 2008). Mean annual precipitation is dominated by snowfall and increases with elevation from about 10 inches per year to more than 60 inches per year. Snowfall tends to be associated with eastern-moving winter storms, which originate from the Pacific Ocean and tend to produce more snow on the windward side of the mountain ranges than on the leeward.

Encompassing much of the western quarter of Colorado from north to south, the Colorado Plateaus Province is located between the Utah border to the west and the Southern Rocky Mountains to the east. The terrain is characterized by mesas and plateaus separated by steep and rugged canyons. Like the Great Plains, the Colorado Plateaus Province has low relative humidity, plentiful sunshine, limited precipitation, and substantial and persistent wind. Climatic variability is extreme over relatively short distances because of the microclimates created by the highly irregular landscape. Below 9,000 feet in elevation, mean annual precipitation ranges from 9 to 18 inches per year, whereas, high elevations (above 9,000 feet) receive in excess of 32 inches per year. Snowfall is a substantial component of annual precipitation at high elevations. However, intense summer thunderstorms can account for 20 to 40 percent of the annual precipitation (Robson and Banta, 1995; Colorado Geological Survey, 2003; Colorado State University, 2008).

\section{Previous Studies}

Many reports document regression equations developed for estimating streamflow statistics in Colorado. Patterson (1964 and 1965), Patterson and Somers (1966), and Matthai (1968) developed regression equations for major river basins in Colorado. Headman and others (1972) developed equations for streamflow statistics based on channel geometry. The first statewide regional regression study, which focused on peak streamflow, was conducted by McCain and Jarrett 
(1976). The hydrologic regions, as described in the section "Definition of Hydrologic Regions of Colorado," originally were defined by McCain and Jarrett (1976) and subsequently adopted by Kircher and others (1985). Kircher and others (1985) developed regional regression equations for a range of high- and low-streamflow characteristics for streams in western Colorado by using USGS streamflow data collected through 1983. Their study is the last regional regression study for nonpeak streamflow statistics in Colorado. Livingston and Minges (1987) developed equations for the estimation of peak streamflows for small drainage basins (less than about 20 square miles) in the Great Plains part of eastern Colorado. Vaill (1999) developed regional regression equations for peak streamflow in Colorado. Vaill (1999) used peak streamflow data collected through 1993 for his study, which represents the most recent regional regression study relative to the current (2009) study for peak streamflow. Other regional regression studies of various streamflow statistics include Waltemeyer (2006) and Thomas and others (1997); however, those studies included only parts of Colorado because of study-specific focus in bordering States.

\section{Regional Regression Equations for Estimation of Natural Streamflow Statistics in Colorado}

The development of regional regression equations for the estimation of natural streamflow statistics in Colorado is composed of four major steps:

1. Definition of distinct hydrologic regions,

2. Selection of stations for inclusion in multilinear regression analysis and distribution of the selected stations into their respective hydrologic regions,

3. Computation of streamflow statistics and basin and climate characteristics for the stations, and

4. Computation of the regression equations for each streamflow characteristic for each hydrologic region as applicable.

These steps are further described in the four sections that follow.

\section{Definition of Hydrologic Regions of Colorado}

For the current study and several previous studies, five hydrologic regions were defined. These are the Mountain, Northwest, Rio Grande, Southwest, and Plains regions (fig. 1). These regions are the same as those originally defined by McCain and Jarrett (1976) and adopted by Kircher and others (1985). A hydrologic region is qualitatively defined as a region of similar hydrology and climatology.

The five hydrologic regions of Colorado were defined on the basis of the physiographic and climatic characteristics that were used to develop best-fit regression equations.
The Mountain region is identified as that region of central Colorado above about 7,500 feet in elevation located between the Colorado-Wyoming border and the Rio Grande basin. The Mountain region encompasses the headwaters of most major river basins in Colorado where the annual peak streamflow generally is produced by snowmelt runoff. The Northwest region is defined as the northwestern part of Colorado below 7,500 feet and encompassing substantial areas of the Yampa, White, and Gunnison River basins. The Rio Grande region ranges in elevation from about 5,000 feet near the Colorado-New Mexico border to more than 14,000 feet in the northern parts and encompasses the Rio Grande basin. The Southwest region is defined as the region located south of the Gunnison River basin and west of the Rio Grande basin and encompasses the Dolores, Animas, and San Juan River basins. The Plains region is east of the Rocky Mountains and below 7,500 feet in the South Platte River basin and below 9,000 feet in the Arkansas River basin.

\section{Selection of Stations}

Various selection criteria were used to develop the list of selected stations for regression analysis reported here. This section details the selection criteria used.

\section{Selection of Stations for Peak- Streamflow Statistics}

A comprehensive list of all USGS streamflow stations within 50 miles of the Colorado border was acquired from the National Water Information System (NWIS) database (U.S. Geological Survey, 2008). From the comprehensive list of candidate stations, those stations with at least 10 years of streamflow record identified as representative of natural streamflow conditions were selected for this study.

Subsequently, multiple stations on the same stream course were evaluated for data similarity as judged by relative drainage-area magnitude. If the relative drainage-area magnitude was less than a factor of 2.5 , data were considered similar. In such cases, stations with the longer applicable record were selected. The reason for excluding stations on the basis of relative drainage-area and stream-course equality enhances the quantity of independent streamflow information (as represented by stations and their respective data). This exclusion process was used in Vaill (1999) and serves the purpose of removing redundant data or hydrologic information from the data base. At the completion of the selection process, 422 stations were identified, with a combined total of 13,054 years of record, and were used in the development of peak-streamflow equations. A map showing the location of the stations is provided in figure 1, and each station, ancillary information, and basin and climatic characteristics are listed in table 1. The "home page" of each station can be accessed online at http://waterdata.usgs.gov/nwis/ nwisman/?site_no $=S T A T I O N \&$ agency_cd $=U S G S$, where the word STATION is replaced by the eight-digit station number. The home pages provide authoritative station names and other identifying characteristics. 
Table 1. Drainage-basin and climatic characteristics at streamflow-gaging stations used for regional regression analysis.

[LATDEG, latitude in decimal degrees; LNGDEG, longitude in decimal degrees; YRSPK, years of record for peak streamflow; YRSANN, years of record for annual streamflow; $A$, drainage area, in square miles; $S$, mean watershed slope, in percent; $P$, mean annual precipitation, in inches; $E$, mean watershed elevation, in feet; $A_{7500}$, percentage of $A$ above 7,500 feet elevation plus $1 ;{ }_{6} P_{100}$, 6-hour, 100-year precipitation, in inches; NA, not applicable, station operated as peak-only or flood-hydrograph station]

\begin{tabular}{|c|c|c|c|c|c|c|c|c|c|c|c|}
\hline $\begin{array}{l}\text { Station } \\
\text { number }\end{array}$ & $\begin{array}{l}\text { Hydrologic } \\
\text { region }\end{array}$ & LATDEG & LNGDEG & YRSPK & YRSANN & $A$ & $S$ & $P$ & $E$ & $A_{7500}$ & ${ }_{6} \boldsymbol{P}_{100}$ \\
\hline 06614800 & Mountain & 40.4961 & 105.8650 & 33 & 34 & 1.5 & 43.2 & 47.1 & 11,246 & 100 & 2.4 \\
\hline 06616000 & Mountain & 40.5494 & 106.0211 & 32 & 32 & 20.5 & 25.7 & 30.9 & 9,794 & 100 & 2.1 \\
\hline 06617100 & Mountain & 40.7411 & 106.2795 & 25 & 24 & 182.1 & 16.8 & 24.4 & 9,275 & 100 & 1.9 \\
\hline 06618500 & Mountain & 40.7264 & 106.2906 & 25 & 24 & 255.4 & 13.7 & 20.5 & 8,994 & 100 & 1.9 \\
\hline 06619000 & Mountain & 40.8616 & 106.3372 & 11 & 10 & 475.9 & 14.1 & 21.3 & 9,027 & 100 & 1.9 \\
\hline 06620400 & Mountain & 41.1833 & 106.2700 & 10 & NA & 22.1 & 8.9 & 31.7 & 9,726 & 100 & 2.1 \\
\hline 06621000 & Mountain & 41.0811 & 106.3075 & 26 & 25 & 118.1 & 12.8 & 23.5 & 9,118 & 100 & 2 \\
\hline 06622000 & Mountain & 41.0500 & 106.5256 & 12 & NA & 107 & 21.7 & 30.4 & 9,168 & 100 & 2.1 \\
\hline 06622500 & Mountain & 41.2083 & 106.5173 & 14 & 13 & 59.8 & 22.6 & 35.5 & 9,484 & 100 & 2.1 \\
\hline 06622700 & Mountain & 41.3702 & 106.5206 & 47 & 47 & 38.1 & 18.5 & 35.3 & 9,403 & 100 & 2.1 \\
\hline 06623800 & Mountain & 41.0236 & 106.8248 & 42 & 43 & 65.8 & 23.2 & 44.3 & 9,457 & 100 & 2.3 \\
\hline 06624500 & Mountain & 41.2139 & 106.7784 & 18 & 15 & 201.5 & 24.3 & 39.5 & 9,048 & 97.8 & 2.2 \\
\hline 06627500 & Northwest & 41.4000 & 107.0006 & 10 & 10 & 34 & 14.3 & 30.3 & 8,384 & 86.6 & 2.1 \\
\hline 06629100 & Northwest & 41.6989 & 106.6267 & 13 & NA & 14.4 & 22.9 & 21.3 & 8,146 & 77.7 & 2 \\
\hline 06630800 & Mountain & 41.6530 & 106.3453 & 13 & NA & 8.9 & 7.6 & 15.8 & 7,759 & 95.6 & 2 \\
\hline 06631000 & Northwest & 41.7166 & 106.3172 & 11 & 9 & 191.4 & 10.9 & 22.4 & 8,319 & 67.7 & 2 \\
\hline 06631100 & Mountain & 41.6400 & 106.3053 & 13 & NA & 25.5 & 11.5 & 23.4 & 8,483 & 99.2 & 2.1 \\
\hline 06632400 & Mountain & 41.5852 & 106.2228 & 41 & 53 & 62.8 & 19.7 & 35.3 & 9,799 & 100 & 2.1 \\
\hline 06696980 & Mountain & 39.3394 & 105.9117 & 14 & 8 & 23.7 & 31.6 & 29 & 11,296 & 100 & 2.8 \\
\hline 06699005 & Mountain & 39.2869 & 105.6958 & 14 & NA & 230.2 & 18.4 & 20.8 & 10,152 & 100 & 2.4 \\
\hline 06700500 & Mountain & 39.2089 & 105.3036 & 58 & NA & 92.6 & 33.7 & 29.6 & 10,054 & 98.5 & 2.6 \\
\hline 06706000 & Mountain & 39.4572 & 105.6586 & 36 & 60 & 126.9 & 34.8 & 24.3 & 11,009 & 100 & 2.4 \\
\hline 06707000 & Plains & 39.4089 & 105.1758 & 60 & NA & 475.7 & 31.7 & 25 & 9,464 & 91 & 2.5 \\
\hline 06709500 & Plains & 39.4844 & 105.0025 & 41 & NA & 302.6 & 18.9 & 21.5 & 6,987 & 23.7 & 3.5 \\
\hline 06710385 & Mountain & 39.6328 & 105.3367 & 23 & NA & 103.2 & 31.9 & 24.8 & 9,606 & 98.1 & 2.9 \\
\hline 06710400 & Mountain & 39.6305 & 105.3217 & 14 & NA & 22.2 & 25.7 & 23.1 & 8,303 & 91.2 & 2.9 \\
\hline 06710600 & Plains & 39.6803 & 105.1978 & 10 & NA & 7.6 & 28.2 & 20.3 & 7,207 & 25.2 & 3.4 \\
\hline 06710990 & Plains & 39.6158 & 105.2322 & 13 & NA & 43.6 & 23.4 & 22.8 & 7,987 & 78 & 3.1 \\
\hline 06711000 & Plains & 39.6355 & 105.1686 & 27 & NA & 50.2 & 25.1 & 22.4 & 7,826 & 69 & 3.1 \\
\hline 06712000 & Plains & 39.3558 & 104.7633 & 67 & NA & 167.9 & 7.3 & 20.6 & 7,093 & 8.2 & 3.5 \\
\hline 06716500 & Mountain & 39.7658 & 105.6261 & 49 & 53 & 146.6 & 44.5 & 27.1 & 11,182 & 100 & 2.4 \\
\hline 06717400 & Mountain & 39.7164 & 105.5714 & 12 & 5 & 43.7 & 36.9 & 25 & 10,642 & 100 & 2.5 \\
\hline 06719500 & Plains & 39.7505 & 105.2489 & 62 & NA & 392.3 & 39.2 & 24.4 & 9,962 & 94.4 & 2.6 \\
\hline 06721500 & Mountain & 40.2189 & 105.5283 & 15 & 16 & 32.5 & 40.8 & 38.2 & 10,825 & 100 & 2.8 \\
\hline 06722500 & Mountain & 40.0908 & 105.5144 & 24 & 24 & 13.4 & 33.6 & 38.3 & 11,119 & 100 & 2.8 \\
\hline 06723000 & Mountain & 40.1667 & 105.4444 & 19 & 5 & 27.8 & 37.3 & 31.4 & 9,959 & 100 & 2.9 \\
\hline 06725500 & Mountain & 39.9617 & 105.5044 & 51 & 87 & 36.5 & 35.3 & 33.2 & 10,345 & 100 & 2.8 \\
\hline 06726900 & Plains & 40.0117 & 105.3486 & 12 & NA & 3.9 & 24.6 & 20.6 & 7,294 & 34.6 & 3 \\
\hline 06729000 & Mountain & 39.9147 & 105.5019 & 7 & 10 & 43.6 & 32.8 & 30.9 & 10,174 & 100 & 2.8 \\
\hline 06730300 & Plains & 39.8778 & 105.2772 & 24 & NA & 15.2 & 36.4 & 23.5 & 8,255 & 89.3 & 3.1 \\
\hline 06732000 & Mountain & 40.3453 & 105.5856 & 18 & 18 & 24.6 & 45.0 & 40.8 & 10,503 & 100 & 2.6 \\
\hline 06739500 & Plains & 40.4539 & 105.1989 & 9 & NA & 134.1 & 29.9 & 20.4 & 7,421 & 46.2 & 3.3 \\
\hline 06748200 & Mountain & 40.5516 & 105.6269 & 13 & 13 & 3.6 & 44.3 & 24.8 & 11,027 & 100 & 2.9 \\
\hline
\end{tabular}


Table 1. Drainage-basin and climatic characteristics at streamflow-gaging stations used for regional regression analysis.—Continued

[LATDEG, latitude in decimal degrees; LNGDEG, longitude in decimal degrees; YRSPK, years of record for peak streamflow; YRSANN, years of record for annual streamflow; $A$, drainage area, in square miles; $S$, mean watershed slope, in percent; $P$, mean annual precipitation, in inches; $E$, mean watershed elevation, in feet; $A_{7500}$, percentage of $A$ above 7,500 feet elevation plus $1 ;{ }_{6} P_{100}, 6$-hour, 100-year precipitation, in inches; NA, not applicable, station operated as peak-only or flood-hydrograph station]

\begin{tabular}{|c|c|c|c|c|c|c|c|c|c|c|c|}
\hline $\begin{array}{l}\text { Station } \\
\text { number }\end{array}$ & $\begin{array}{l}\text { Hydrologic } \\
\text { region }\end{array}$ & LATDEG & LNGDEG & YRSPK & YRSANN & $A$ & $S$ & $P$ & $E$ & $A_{7500}$ & ${ }_{6} \boldsymbol{P}_{100}$ \\
\hline 06748510 & Mountain & 40.6386 & 105.6617 & 13 & 13 & 0.9 & 22.4 & 24 & 10,878 & 100 & 2.8 \\
\hline 06748530 & Mountain & 40.6230 & 105.5650 & 13 & 13 & 12.4 & 26.7 & 20 & 9,833 & 100 & 2.8 \\
\hline 06748600 & Mountain & 40.6469 & 105.4936 & 23 & 23 & 92.6 & 28.9 & 21.3 & 9,866 & 100 & 2.8 \\
\hline 06755000 & Plains & 41.1264 & 105.1944 & 35 & 21 & 13.5 & 10.4 & 18.9 & 7,808 & 89.6 & 3 \\
\hline 06758200 & Plains & 39.3372 & 104.4755 & 10 & NA & 115.4 & 7.0 & 19.8 & 7,002 & 5.6 & 3.5 \\
\hline 06758250 & Plains & 39.6130 & 104.4508 & 10 & NA & 6.5 & 5.7 & 18.1 & 5,923 & 0 & 3.3 \\
\hline 06758700 & Plains & 39.4925 & 104.1633 & 10 & NA & 2.7 & 9.2 & 17.3 & 5,849 & 0 & 3.3 \\
\hline 06761900 & Plains & 41.2564 & 104.0811 & 22 & NA & 0.6 & 1.7 & 15.5 & 5,300 & 0 & 3.4 \\
\hline 06762500 & Plains & 41.2278 & 103.8931 & 64 & NA & $1,216.1$ & 4.0 & 15.6 & 5,850 & 3.8 & 3.1 \\
\hline 06762600 & Plains & 41.3197 & 104.0808 & 25 & NA & 7.6 & 2.0 & 16.8 & 5,339 & 0 & 3.4 \\
\hline 06821300 & Plains & 39.5200 & 103.4436 & 11 & NA & 6.8 & 2.6 & 16.3 & 5,348 & 0 & 3.7 \\
\hline 06821400 & Plains & 39.9067 & 102.2694 & 11 & NA & 19.8 & 2.2 & 18.2 & 3,899 & 0 & 4.6 \\
\hline 06823500 & Plains & 40.0394 & 101.8667 & 65 & NA & 176.8 & 4.3 & 18.3 & 3,654 & 0 & 4.4 \\
\hline 06825500 & Plains & 39.5755 & 102.2521 & 26 & NA & 268.9 & 3.0 & 17.7 & 4,333 & 0 & 4.5 \\
\hline 06844700 & Plains & 39.2853 & 101.4660 & 22 & NA & 85.7 & 1.6 & 19.5 & 3,619 & 0 & 4.4 \\
\hline 06844800 & Plains & 39.3206 & 101.6329 & 33 & NA & 20.1 & 1.0 & 19.5 & 3,698 & 0 & 4.4 \\
\hline 06858500 & Plains & 39.0179 & 101.3479 & 33 & NA & 696.2 & 2.5 & 18 & 3,964 & 0 & 4.6 \\
\hline 07079500 & Mountain & 39.2597 & 106.3406 & 11 & 11 & 51.2 & 33.4 & 25.3 & 11,433 & 100 & 2.2 \\
\hline 07081000 & Mountain & 39.2642 & 106.3409 & 14 & 11 & 45.7 & 22.8 & 22 & 10,800 & 100 & 2.2 \\
\hline 07082000 & Mountain & 39.2697 & 106.3950 & 8 & 21 & 23.8 & 32.8 & 30.8 & 11,146 & 100 & 2.5 \\
\hline 07083000 & Mountain & 39.1722 & 106.3892 & 60 & 61 & 23.5 & 49.0 & 34.5 & 11,955 & 100 & 2.7 \\
\hline 07089000 & Mountain & 38.8128 & 106.2222 & 48 & NA & 68.5 & 45.8 & 22.2 & 11,380 & 100 & 2.7 \\
\hline 07091000 & Mountain & 38.7336 & 106.1600 & 20 & NA & 83 & 47.1 & 22.5 & 11,379 & 100 & 2.7 \\
\hline 07093500 & Mountain & 38.5214 & 105.9897 & 14 & NA & 211.6 & 36.1 & 18.3 & 10,096 & 98.3 & 2.5 \\
\hline 07093775 & Plains & 38.4672 & 105.8600 & 23 & NA & 211 & 22.7 & 13.8 & 9,356 & 97.2 & 2.4 \\
\hline 07095000 & Plains & 38.1861 & 105.4836 & 66 & NA & 320.5 & 19.5 & 21.2 & 9,189 & 100 & 2.7 \\
\hline 07096500 & Plains & 38.4364 & 105.1914 & 32 & NA & 436.3 & 26.2 & 16.7 & 8,428 & 81.4 & 2.4 \\
\hline 07099100 & Plains & 38.3742 & 104.9642 & 11 & NA & 213.8 & 27.9 & 20.4 & 8,143 & 51.7 & 3.3 \\
\hline 07099230 & Plains & 38.4650 & 104.8272 & 28 & NA & 62.2 & 25.1 & 19.2 & 6,860 & 23.6 & 3.6 \\
\hline 07105920 & Plains & 38.6817 & 104.8586 & 12 & NA & 11 & 48.7 & 24 & 8,832 & 89.1 & 3.6 \\
\hline 07105945 & Plains & 38.7075 & 104.8466 & 28 & NA & 6.8 & 48.5 & 22.5 & 8,193 & 75.7 & 3.6 \\
\hline 07107500 & Plains & 38.0517 & 104.7936 & 18 & NA & 171.4 & 28.6 & 22.1 & 7,964 & 52.9 & 3.6 \\
\hline 07114000 & Rio Grande & 37.4200 & 105.0528 & 46 & NA & 53.1 & 35.4 & 27.3 & 9,883 & 100 & 3.3 \\
\hline 07118000 & Plains & 37.3864 & 104.6658 & 11 & NA & 140.1 & 27.0 & 22 & 8,062 & 60 & 3.5 \\
\hline 07120620 & Plains & 37.5547 & 104.0216 & 21 & NA & 15.6 & 6.2 & 14.7 & 5,582 & 0 & 4 \\
\hline 07121500 & Plains & 38.0031 & 103.6561 & 41 & NA & 510.9 & 3.9 & 13.6 & 4,871 & 0 & 4 \\
\hline 07124300 & Plains & 37.1147 & 104.6053 & 18 & NA & 100.1 & 25.5 & 19.5 & 7,366 & 42.7 & 3.9 \\
\hline 07125100 & Plains & 37.2000 & 104.1941 & 13 & NA & 79.1 & 10.0 & 15.6 & 6,077 & 6.5 & 3.9 \\
\hline 07125500 & Plains & 37.1684 & 104.1450 & 14 & NA & 162.2 & 13.5 & 18 & 6,642 & 24.7 & 4.1 \\
\hline 07126100 & Plains & 37.3045 & 104.0155 & 16 & NA & 84.8 & 2.8 & 14 & 5,479 & 0 & 3.8 \\
\hline 07126200 & Plains & 37.3456 & 103.9580 & 41 & NA & 161.4 & 2.8 & 14.3 & 5,521 & 0 & 3.8 \\
\hline 07126325 & Plains & 37.4242 & 103.9202 & 24 & NA & 48.7 & 4.4 & 14.1 & 5,330 & 0 & 4.1 \\
\hline 07126390 & Plains & 37.4928 & 103.8280 & 21 & NA & 48.6 & 4.6 & 13.9 & 5,161 & 0 & 4.2 \\
\hline
\end{tabular}


Table 1. Drainage-basin and climatic characteristics at streamflow-gaging stations used for regional regression analysis.-Continued

[LATDEG, latitude in decimal degrees; LNGDEG, longitude in decimal degrees; YRSPK, years of record for peak streamflow; YRSANN, years of record for annual streamflow; $A$, drainage area, in square miles; $S$, mean watershed slope, in percent; $P$, mean annual precipitation, in inches; $E$, mean watershed elevation, in feet; $A_{7500}$, percentage of $A$ above 7,500 feet elevation plus 1; ${ }_{6}{ }_{100}, 6$, hour, 100-year precipitation, in inches; NA, not applicable, station operated as peak-only or flood-hydrograph station]

\begin{tabular}{|c|c|c|c|c|c|c|c|c|c|c|c|}
\hline $\begin{array}{l}\text { Station } \\
\text { number }\end{array}$ & $\begin{array}{l}\text { Hydrologic } \\
\text { region }\end{array}$ & LATDEG & LNGDEG & YRSPK & YRSANN & $A$ & $S$ & $\boldsymbol{P}$ & $E$ & $A_{7500}$ & ${ }_{6} P_{100}$ \\
\hline 07126415 & Plains & 37.5153 & 103.7255 & 23 & NA & 48.7 & 11.2 & 13.9 & 4,981 & 0 & 4.3 \\
\hline 07126470 & Plains & 37.5439 & 103.6322 & 16 & NA & 421.3 & 8.6 & 15.6 & 5,607 & 0 & 4.5 \\
\hline 07126480 & Plains & 37.5892 & 103.6483 & 21 & NA & 56.1 & 11.3 & 14.1 & 4,968 & 0 & 4.3 \\
\hline 07133200 & Plains & 37.7242 & 102.7405 & 11 & NA & 2.4 & 5.2 & 16 & 4,431 & 0 & 5 \\
\hline 07138600 & Plains & 38.5250 & 101.6216 & 39 & NA & 25.2 & 1.1 & 17.7 & 3,650 & 0 & 4.4 \\
\hline 07138650 & Plains & 38.4811 & 101.4882 & 20 & NA & 500.3 & 1.4 & 17 & 3,858 & 0 & 4.7 \\
\hline 07153500 & Plains & 36.9875 & 103.4241 & 33 & NA & 529.2 & 13.5 & 17.2 & 6,251 & 3.4 & 4.1 \\
\hline 07154400 & Plains & 36.8833 & 103.0183 & 53 & NA & 112.2 & 11.8 & 16.7 & 5,087 & 0 & 4.4 \\
\hline 07154650 & Plains & 36.8978 & 102.9016 & 22 & NA & 24.9 & 12.0 & $\begin{array}{l}10.1 \\
17.2\end{array}$ & 4,555 & 0 & 4.2 \\
\hline 07155100 & Plains & 36.7722 & 102.8049 & 18 & NA & 10.6 & 1.8 & 17.1 & 4,624 & 0 & 4.2 \\
\hline 07155590 & Plains & 37.1220 & 101.8979 & 35 & NA & $2,926.6$ & 8.4 & 17.0 & 5,001 & 0.8 & 4.6 \\
\hline 07155900 & Plains & 37.1909 & 101.8988 & 34 & NA & 57.1 & 0.6 & 17.3 & 3,669 & 0 & 4.9 \\
\hline 07156010 & Plains & 37.2584 & 101.7754 & 15 & NA & 411.3 & 0.9 & 17.2 & 3,946 & 0 & 5.1 \\
\hline 07156100 & Plains & 37.5000 & 101.7616 & 15 & NA & 584.2 & 1.5 & 17 & 4,411 & 0 & 5.1 \\
\hline 07156220 & Plains & 37.6264 & 101.7616 & 32 & NA & 813.3 & 1.6 & 17 & 4,149 & 0 & 5.1 \\
\hline 07156600 & Plains & 37.3353 & 101.0504 & 33 & NA & 21.5 & 1.7 & 18.6 & 2,983 & 0 & 4.8 \\
\hline 07199000 & Plains & 36.7872 & 104.4622 & 40 & NA & 199.1 & 23.6 & 20.1 & 7,594 & 59.1 & 3.6 \\
\hline 07201000 & Plains & 36.9058 & 104.4361 & 48 & NA & 14.6 & 23.8 & 20.9 & 7,608 & 50.8 & 3.9 \\
\hline 07201200 & Plains & 36.8308 & 104.3333 & 30 & NA & 5.2 & 16.2 & 19 & 7,120 & 24 & 3.9 \\
\hline 07203000 & Plains & 36.6817 & 104.7867 & 76 & NA & 295.2 & 23.7 & 20.6 & 8,016 & 70.7 & 3.3 \\
\hline 07204000 & Rio Grande & 36.5539 & 105.2678 & 69 & NA & 78.8 & 26.3 & 21.1 & 9,405 & 100 & 3.3 \\
\hline 07204500 & Rio Grande & 36.4853 & 105.2656 & 68 & NA & 73.2 & 19.4 & 23.6 & 9,151 & 100 & 3 \\
\hline 07205000 & Rio Grande & 36.5186 & 105.2753 & 72 & NA & 10.5 & 28.1 & 21.7 & 9,405 & 100 & 3.1 \\
\hline 07206400 & Rio Grande & 36.5264 & 105.1750 & 40 & NA & 7.2 & 48.2 & 25.9 & 9,810 & 100 & 3.3 \\
\hline 07207500 & Plains & 36.5739 & 104.9461 & 57 & NA & 185.4 & 27.4 & 21.5 & 8,589 & 90.4 & 3.3 \\
\hline 07208500 & Plains & 36.3725 & 104.9689 & 83 & NA & 60.1 & 24.2 & 26.2 & 9,550 & 97.1 & 3.5 \\
\hline 07211000 & Plains & 36.3603 & 104.5986 & 75 & NA & 949 & 21.6 & 20.7 & 8,106 & 65.6 & 3.3 \\
\hline 07225500 & Plains & 36.3259 & 103.9255 & 12 & NA & 268.7 & 6.2 & 17.2 & 6,532 & 2.3 & 4.1 \\
\hline 07227295 & Plains & 36.3889 & 103.3186 & 42 & NA & 1.2 & 2.0 & 16.2 & 5,182 & 0 & 4.6 \\
\hline 07232500 & Plains & 36.7214 & 101.4896 & 57 & NA & 2,033 & 3.0 & 17.3 & 4,463 & 0.1 & 4.5 \\
\hline 07232650 & Plains & 36.5564 & 102.7866 & 12 & NA & 33.9 & 1.1 & 16.6 & 4,593 & 0 & 4.3 \\
\hline 08216500 & Rio Grande & 37.8561 & 106.9275 & 32 & 31 & 34.2 & $\begin{array}{r}1.1 \\
40.5\end{array}$ & 30.6 & 11,473 & 100 & 2.3 \\
\hline 08218500 & Rio Grande & 37.7519 & 106.8300 & 36 & 37 & 91.9 & 35.7 & 37.4 & 10,723 & 100 & 2.4 \\
\hline 08219500 & Rio Grande & 37.6569 & 106.6492 & 80 & 80 & 210.6 & 31.3 & 38.2 & 10,398 & 100 & 2.7 \\
\hline 08220500 & Rio Grande & 37.5917 & 106.4500 & 47 & 38 & $\begin{array}{r}210.0 \\
52.7\end{array}$ & 30.7 & $\begin{array}{l}30.2 \\
33.6\end{array}$ & 10,507 & 100 & 2.7 \\
\hline 08223500 & Rio Grande & 37.4903 & 106.2595 & 25 & 23 & 30.7 & 34.6 & 31.8 & 10,356 & 100 & 2.5 \\
\hline 08224500 & Rio Grande & 38.2203 & 106.0897 & 59 & 58 & $\begin{array}{l}50.1 \\
45.4\end{array}$ & $\begin{array}{l}34.0 \\
35.8\end{array}$ & $\begin{array}{l}31.0 \\
18.8\end{array}$ & 10,405 & 100 & 2.3 \\
\hline 08227000 & Rio Grande & 38.1633 & 106.2906 & 87 & 87 & 516.9 & 22.8 & 20.8 & 9,949 & 100 & 2.1 \\
\hline 08227500 & Rio Grande & 38.0136 & 105.6928 & 46 & 34 & 12.9 & 64.6 & 30.1 & 11,294 & 100 & 2.6 \\
\hline 08230500 & Rio Grande & 37.8597 & 106.3195 & 57 & 39 & 106.2 & 27.6 & 26.5 & 10,056 & 100 & 2.2 \\
\hline 08231000 & Rio Grande & 37.8133 & 106.3186 & 68 & 47 & 62.2 & 27.8 & 31.1 & 10,259 & 100 & 2.3 \\
\hline 08236000 & Rio Grande & 37.3747 & 106.3348 & 54 & 65 & 107.6 & 33.7 & 38.4 & 10,872 & 100 & 2.6 \\
\hline
\end{tabular}


Table 1. Drainage-basin and climatic characteristics at streamflow-gaging stations used for regional regression analysis.—Continued

[LATDEG, latitude in decimal degrees; LNGDEG, longitude in decimal degrees; YRSPK, years of record for peak streamflow; YRSANN, years of record for annual streamflow; $A$, drainage area, in square miles; $S$, mean watershed slope, in percent; $P$, mean annual precipitation, in inches; $E$, mean watershed elevation, in feet; $A_{7500}$, percentage of $A$ above 7,500 feet elevation plus $1 ;{ }_{6} P_{100}, 6$-hour, 100-year precipitation, in inches; NA, not applicable, station operated as peak-only or flood-hydrograph station]

\begin{tabular}{|c|c|c|c|c|c|c|c|c|c|c|c|}
\hline $\begin{array}{l}\text { Station } \\
\text { number }\end{array}$ & $\begin{array}{l}\text { Hydrologic } \\
\text { region }\end{array}$ & LATDEG & LNGDEG & YRSPK & YRSANN & $\boldsymbol{A}$ & $S$ & $\boldsymbol{P}$ & $E$ & $A_{7500}$ & ${ }_{6} \boldsymbol{P}_{100}$ \\
\hline 08240500 & Rio Grande & 37.3747 & 105.2950 & 59 & 48 & 52.7 & 41.2 & 23.2 & 10,492 & 100 & 2.6 \\
\hline 08241500 & Rio Grande & 37.4250 & 105.4150 & 58 & 37 & 182.9 & 25.1 & 19.4 & 9,169 & 100 & 2.5 \\
\hline 08242500 & Rio Grande & 37.4472 & 105.4258 & 67 & 67 & 40.2 & 32.5 & 22.3 & 10,152 & 100 & 2.7 \\
\hline 08245500 & Rio Grande & 37.3539 & 106.5250 & 14 & 17 & 44.2 & 35.0 & 45 & 11,218 & 100 & 2.9 \\
\hline 08246500 & Rio Grande & 37.0539 & 106.1875 & 43 & 97 & 281.6 & 27.8 & 35.3 & 10,481 & 100 & 2.5 \\
\hline 08247500 & Rio Grande & 36.9931 & 106.0386 & 82 & 67 & 115.7 & 14.6 & 25.2 & 9,353 & 100 & 3.4 \\
\hline 08248000 & Rio Grande & 36.9822 & 106.0736 & 88 & 88 & 152.8 & 21.7 & 30.6 & 9,861 & 100 & 3.1 \\
\hline 08248500 & Rio Grande & 37.1770 & 105.8781 & 59 & 59 & 372.9 & 15.2 & 23.5 & 9,192 & 100 & 2.9 \\
\hline 08252500 & Rio Grande & 36.8983 & 105.2544 & 68 & NA & 25.6 & 23.5 & 26.9 & 10,599 & 100 & 3.3 \\
\hline 08253000 & Rio Grande & 36.8969 & 105.2603 & 70 & NA & 18.5 & 29.9 & 28.7 & 11,023 & 100 & 3.5 \\
\hline 08253500 & Rio Grande & 36.8842 & 105.2811 & 69 & NA & 2.2 & 39.2 & 29.6 & 11,313 & 100 & 3.5 \\
\hline 08255000 & Rio Grande & 36.9528 & 105.4103 & 10 & NA & 11.2 & 35.0 & 27 & 10,929 & 100 & 3.6 \\
\hline 08263000 & Rio Grande & 36.8292 & 105.5478 & 32 & 25 & 10.6 & 41.7 & 28.9 & 10,973 & 100 & 3.4 \\
\hline 08264000 & Rio Grande & 36.6223 & 105.3895 & 24 & 14 & 19.2 & 37.6 & 30.2 & 11,077 & 100 & 3.5 \\
\hline 08265000 & Rio Grande & 36.7034 & 105.5686 & 75 & 77 & 112.2 & 42.4 & 26.3 & 10,149 & 100 & 3.3 \\
\hline 08267500 & Rio Grande & 36.5419 & 105.5561 & 70 & 73 & 37.1 & 55.2 & 28.3 & 10,389 & 100 & 3.3 \\
\hline 08268500 & Rio Grande & 36.5322 & 105.6856 & 51 & 67 & 66.6 & 43.1 & 23.3 & 9,401 & 83.7 & 3.1 \\
\hline 08269000 & Rio Grande & 36.4394 & 105.5036 & 72 & 56 & 63 & 40.2 & 25.1 & 9,608 & 99.8 & 3 \\
\hline 08275000 & Rio Grande & 36.3756 & 105.5492 & 19 & 17 & 60.8 & 31.2 & 24.6 & 9,040 & 98.9 & 2.9 \\
\hline 08275500 & Rio Grande & 36.2989 & 105.5817 & 50 & 52 & 80.4 & 32.7 & 27.7 & 9,352 & 97.7 & 3.3 \\
\hline 08275600 & Rio Grande & 36.3320 & 105.5789 & 24 & 23 & 38 & 33.5 & 26.6 & 9,362 & 99.4 & 3.2 \\
\hline 08281200 & Rio Grande & 36.9556 & 106.5367 & 13 & NA & 28.1 & 25.8 & 34.2 & 9,724 & 100 & 2.9 \\
\hline 08284000 & Rio Grande & 36.6986 & 106.5575 & 24 & NA & 50.6 & 15.6 & 26.1 & 8,856 & 99.9 & 2.9 \\
\hline 08284100 & Rio Grande & 36.6625 & 106.6331 & 51 & 52 & 475.3 & 20.2 & 30.2 & 9,304 & 94.4 & 2.9 \\
\hline 08284500 & Rio Grande & 36.6681 & 106.7048 & 34 & 28 & 192.5 & 13.6 & 20 & 7,792 & 75.3 & 2.5 \\
\hline 08286650 & Rio Grande & 36.3172 & 106.4853 & 33 & NA & 143.9 & 15.6 & 18.9 & 8,140 & 77.7 & 2.8 \\
\hline 08288000 & Rio Grande & 36.3917 & 106.2395 & 33 & 19 & 48 & 20.2 & 24.6 & 9,157 & 99.9 & 3.2 \\
\hline 08289000 & Rio Grande & 36.3497 & 106.0442 & 75 & 75 & 411.9 & 20.2 & 19.7 & 8,492 & 80.5 & 2.9 \\
\hline 09012500 & Mountain & 40.2533 & 105.8114 & 10 & 8 & 45.8 & 42.5 & 36.8 & 10,680 & 100 & 2.1 \\
\hline 09016500 & Mountain & 40.1125 & 105.7497 & 27 & 28 & 47.8 & 50.2 & 35.8 & 10,641 & 100 & 2.3 \\
\hline 09020000 & Mountain & 40.1805 & 106.0092 & 19 & 18 & 109.2 & 29.5 & 25.8 & 9,630 & 100 & 2.1 \\
\hline 09024000 & Mountain & 39.9000 & 105.7767 & 20 & 97 & 27.8 & 38.9 & 32.8 & 10,767 & 100 & 2.3 \\
\hline 09026500 & Mountain & 39.9100 & 105.8783 & 33 & 73 & 32.8 & 36.3 & 29 & 10,738 & 100 & 2.2 \\
\hline 09032000 & Mountain & 39.9500 & 105.7656 & 14 & 73 & 19.9 & 29.7 & 30.5 & 10,453 & 100 & 2.3 \\
\hline 09032500 & Mountain & 39.9975 & 105.8236 & 17 & 26 & 51.1 & 27.0 & 28.7 & 9,973 & 100 & 2.2 \\
\hline 09033000 & Mountain & 40.0508 & 105.7775 & 21 & 19 & 8.1 & 23.3 & 34.8 & 10,509 & 100 & 2.2 \\
\hline 09033500 & Mountain & 40.0867 & 105.7947 & 10 & 9 & 11.7 & 29.3 & 26.5 & 9,538 & 100 & 2 \\
\hline 09034000 & Mountain & 40.0853 & 105.9553 & 5 & 23 & 295.9 & 26.6 & 25.8 & 9,756 & 100 & 2.1 \\
\hline 09034500 & Mountain & 40.0833 & 106.0881 & 28 & 86 & 823.9 & 29.7 & 26.7 & 9,737 & 100 & 2.1 \\
\hline 09034900 & Mountain & 39.7603 & 105.9064 & 41 & 42 & 5.5 & 49.7 & 32.3 & 11,793 & 100 & 2.3 \\
\hline 09035500 & Mountain & 39.7789 & 105.9283 & 28 & 50 & 16.4 & 46.0 & 30.7 & 11,559 & 100 & 2.3 \\
\hline 09035700 & Mountain & 39.7972 & 106.0261 & 32 & 42 & 35.2 & 42.1 & 28.6 & 11,106 & 100 & 2.2 \\
\hline
\end{tabular}


Table 1. Drainage-basin and climatic characteristics at streamflow-gaging stations used for regional regression analysis.-Continued

[LATDEG, latitude in decimal degrees; LNGDEG, longitude in decimal degrees; YRSPK, years of record for peak streamflow; YRSANN, years of record for annual streamflow; $A$, drainage area, in square miles; $S$, mean watershed slope, in percent; $P$, mean annual precipitation, in inches; $E$, mean watershed elevation, in feet; $A_{7500}$, percentage of $A$ above 7,500 feet elevation plus $1 ;{ }_{6} P_{100}$, 6-hour, 100-year precipitation, in inches; NA, not applicable, station operated as peak-only or flood-hydrograph station]

\begin{tabular}{|c|c|c|c|c|c|c|c|c|c|c|c|}
\hline $\begin{array}{l}\text { Station } \\
\text { number }\end{array}$ & $\begin{array}{l}\text { Hydrologic } \\
\text { region }\end{array}$ & LATDEG & LNGDEG & YRSPK & YRSANN & $A$ & $S$ & $P$ & $E$ & $A_{7500}$ & ${ }_{6} P_{100}$ \\
\hline 09035800 & Mountain & 39.8005 & 106.0264 & 41 & 42 & 8.8 & 37.5 & 28.3 & 10,945 & 100 & 2.2 \\
\hline 09035900 & Mountain & 39.7958 & 106.0306 & 41 & 42 & 27.4 & 42.1 & 28.7 & 10,989 & 100 & 2.3 \\
\hline 09036500 & Mountain & 39.9075 & 106.0172 & 10 & 10 & 13.9 & 36.8 & 28.2 & 10,526 & 100 & 2.1 \\
\hline 09039000 & Mountain & 40.2175 & 106.3131 & 40 & 40 & 44.6 & 36.7 & 28.3 & 9,869 & 100 & 2.2 \\
\hline 09040000 & Mountain & 40.1575 & 106.2834 & 37 & 36 & 75.7 & 30.0 & 23.2 & 9,266 & 100 & 2.2 \\
\hline 09041100 & Mountain & 40.2405 & 106.3736 & 13 & 13 & 11.5 & 25.2 & 20.6 & 8,907 & 100 & 2 \\
\hline 09046530 & Mountain & 39.4930 & 106.0447 & 10 & 8 & 11.1 & 34.2 & 28 & 11,034 & 100 & 2.2 \\
\hline 09047000 & Mountain & 39.6139 & 106.0520 & 44 & 50 & 128.2 & 31.8 & 27 & 10,913 & 100 & 2.3 \\
\hline 09047500 & Mountain & 39.6055 & 105.9431 & 58 & 58 & 57.8 & 41.9 & 29.8 & 11,497 & 100 & 2.4 \\
\hline 09047700 & Mountain & 39.5944 & 105.9725 & 49 & 50 & 9.1 & 35.2 & 26.2 & 10,879 & 100 & 2.1 \\
\hline 09050100 & Mountain & 39.5753 & 106.1106 & 49 & 50 & 92.2 & 35.3 & 29 & 11,238 & 100 & 2.3 \\
\hline 09052400 & Mountain & 39.7280 & 106.1734 & 26 & 28 & 8.6 & 48.9 & 31.8 & 11,199 & 100 & 2.4 \\
\hline 09052800 & Mountain & 39.7630 & 106.1925 & 27 & 28 & 14 & 52.8 & 32.5 & 11,029 & 100 & 2.3 \\
\hline 09055000 & Mountain & 39.8528 & 106.2678 & 10 & 9 & 7.6 & 25.8 & 24.4 & 9,600 & 100 & 2.1 \\
\hline 09058610 & Mountain & 39.7039 & 106.4575 & 33 & 33 & 3.3 & 28.2 & 28.9 & 10,024 & 100 & 2.2 \\
\hline 09058700 & Mountain & 39.6983 & 106.4456 & 40 & 40 & 3 & 22.5 & 27.6 & 9,848 & 100 & 2.2 \\
\hline 09058800 & Mountain & 39.7317 & 106.4267 & 38 & 40 & 3.6 & 28.2 & 29.6 & 10,575 & 100 & 2.4 \\
\hline 09059500 & Northwest & 39.8000 & 106.5839 & 62 & 63 & 93.4 & 33.0 & 25.8 & 9,722 & 99.1 & 2.2 \\
\hline 09060500 & Mountain & 40.0411 & 106.6559 & 28 & 28 & 47.8 & 18.8 & 29.3 & 9,390 & 100 & 2.2 \\
\hline 09063200 & Mountain & 39.5222 & 106.3236 & 40 & 43 & 9.5 & 34.0 & 26.7 & 10,823 & 100 & 2.2 \\
\hline 09063400 & Mountain & 39.5228 & 106.3361 & 43 & 44 & 23.8 & 34.2 & 28.1 & 10,721 & 100 & 2.2 \\
\hline 09064500 & Mountain & 39.4733 & 106.3678 & 32 & 71 & 58.2 & 38.0 & 27.8 & 10,922 & 100 & 2.4 \\
\hline 09065100 & Mountain & 39.5680 & 106.4125 & 46 & 47 & 34.2 & 47.0 & 30.8 & 11,184 & 100 & 2.3 \\
\hline 09065500 & Mountain & 39.6258 & 106.2781 & 50 & 53 & 14.5 & 50.5 & 30.5 & 11,116 & 100 & 2.4 \\
\hline 09066000 & Mountain & 39.5964 & 106.2650 & 51 & 53 & 12.5 & 31.7 & 28 & 10,713 & 100 & 2.3 \\
\hline 09066100 & Mountain & 39.6400 & 106.2934 & 40 & 44 & 4.5 & 60.1 & 31.4 & 11,088 & 100 & 2.3 \\
\hline 09066150 & Mountain & 39.6436 & 106.3025 & 33 & 41 & 5.3 & 58.0 & 30.6 & 11,074 & 100 & 2.4 \\
\hline 09066200 & Mountain & 39.6483 & 106.3231 & 40 & 43 & 6.1 & 53.5 & 28.5 & 10,904 & 100 & 2.4 \\
\hline 09066300 & Mountain & 39.6458 & 106.3823 & 42 & 43 & 5.9 & 36.9 & 25.7 & 10,504 & 100 & 2.3 \\
\hline 09066400 & Mountain & 39.6828 & 106.4014 & 43 & 44 & 7.4 & 30.7 & 26.6 & 10,399 & 100 & 2.3 \\
\hline 09066500 & Mountain & 39.6147 & 106.4400 & 12 & 12 & 101 & 40.6 & 27.9 & 10,305 & 100 & 2.2 \\
\hline 09067005 & Mountain & 39.6317 & 106.5225 & 11 & 11 & 395.1 & 37.1 & 26.7 & 10,456 & 99.9 & 2.2 \\
\hline 09068000 & Mountain & 39.5572 & 106.7631 & 22 & 22 & 71.5 & 39.5 & 26.1 & 9,951 & 99.9 & 2.2 \\
\hline 09069500 & Mountain & 39.5455 & 106.9348 & 11 & 12 & 62.6 & 35.4 & 24.7 & 9,689 & 99.8 & 2.2 \\
\hline 09070000 & Mountain & 39.6494 & 106.9537 & 60 & 61 & 944.6 & 33.6 & 23.1 & 9,481 & 88.3 & 2.1 \\
\hline 09071300 & Mountain & 39.7166 & 107.3103 & 20 & 20 & 6.5 & 9.6 & 42.7 & 10,771 & 100 & 3 \\
\hline 09073500 & Mountain & 39.1894 & 106.8145 & 13 & 43 & 107.4 & 42.0 & 28.6 & 11,215 & 100 & 2.4 \\
\hline 09073700 & Mountain & 39.2139 & 106.6559 & 16 & 16 & 6.1 & 47.9 & 31.9 & 11,762 & 100 & 2.5 \\
\hline 09073800 & Mountain & 39.1964 & 106.6900 & 10 & 10 & 8.6 & 36.7 & 29.9 & 11,439 & 100 & 2.4 \\
\hline 09073900 & Mountain & 39.1889 & 106.7184 & 10 & 10 & 6.6 & 28.1 & 28.9 & 11,263 & 100 & 2.3 \\
\hline 09074000 & Mountain & 39.2058 & 106.7975 & 43 & 44 & 42.1 & 34.1 & 28.8 & 10,890 & 100 & 2.3 \\
\hline 09074800 & Mountain & 39.0875 & 106.8123 & 25 & 25 & 32.3 & 46.9 & 36.4 & 11,431 & 100 & 2.4 \\
\hline
\end{tabular}


[LATDEG, latitude in decimal degrees; LNGDEG, longitude in decimal degrees; YRSPK, years of record for peak streamflow; YRSANN, years of record for annual streamflow; $A$, drainage area, in square miles; $S$, mean watershed slope, in percent; $P$, mean annual precipitation, in inches; $E$, mean watershed elevation, in feet; $A_{7500}$, percentage of $A$ above 7,500 feet elevation plus $1 ;{ }_{6} P_{100}, 6$-hour, 100-year precipitation, in inches; NA, not applicable, station operated as peak-only or flood-hydrograph station]

\begin{tabular}{|c|c|c|c|c|c|c|c|c|c|c|c|}
\hline $\begin{array}{l}\text { Station } \\
\text { number }\end{array}$ & $\begin{array}{c}\text { Hydrologic } \\
\text { region }\end{array}$ & LATDEG & LNGDEG & YRSPK & YRSANN & $A$ & $S$ & $P$ & $E$ & $A_{7500}$ & ${ }_{6} \boldsymbol{P}_{100}$ \\
\hline 09075700 & Mountain & 39.1236 & 106.9053 & 25 & 25 & 35.3 & 60.2 & 44.3 & 11,384 & 100 & 2.4 \\
\hline 09077200 & Mountain & 39.2455 & 106.5314 & 9 & 19 & 18.9 & 53.4 & 36.1 & 11,846 & 100 & 2.7 \\
\hline 09077800 & Mountain & 39.2417 & 106.5931 & 9 & 19 & 11.5 & 48.4 & 33.2 & 11,598 & 100 & 2.6 \\
\hline 09078000 & Mountain & 39.3308 & 106.6581 & 28 & 41 & 88.9 & 41.0 & 31.6 & 10,975 & 100 & 2.5 \\
\hline 09078100 & Mountain & 39.3589 & 106.5684 & 15 & 17 & 10.7 & 48.2 & 32.6 & 11,204 & 100 & 2.5 \\
\hline 09078200 & Mountain & 39.3342 & 106.5753 & 16 & 17 & 7.2 & 38.0 & 31.9 & 10,891 & 100 & 2.5 \\
\hline 09078500 & Mountain & 39.3428 & 106.6659 & 39 & 41 & 42.3 & 37.7 & 29.5 & 10,546 & 100 & 2.5 \\
\hline 09080300 & Mountain & 39.3617 & 106.8206 & 14 & 14 & 12.4 & 44.0 & 26.8 & 10,103 & 100 & 2.3 \\
\hline 09081600 & Northwest & 39.2322 & 107.2273 & 50 & 52 & 167.3 & 49.4 & 39.5 & 10,166 & 97.7 & 2.3 \\
\hline 09082800 & Mountain & 39.3297 & 107.3334 & 16 & 16 & 27.9 & 22.1 & 34 & 9,463 & 100 & 2.4 \\
\hline 09083000 & Northwest & 39.3305 & 107.2245 & 14 & 14 & 75.4 & 30.9 & 32.6 & 9,168 & 94.7 & 2.3 \\
\hline 09084000 & Mountain & 39.4667 & 107.0523 & 15 & 15 & 30.3 & 30.4 & 27 & 9,490 & 100 & 2.2 \\
\hline 09085200 & Northwest & 39.6053 & 107.4484 & 18 & 17 & 23.8 & 38.1 & 35.8 & 9,716 & 87.6 & 2.7 \\
\hline 09085300 & Northwest & 39.6091 & 107.4348 & 15 & 14 & 15.8 & 34.9 & 34.1 & 9,661 & 90.9 & 2.5 \\
\hline 09085400 & Northwest & 39.5978 & 107.4239 & 14 & 13 & 6.2 & 40.6 & 28.9 & 9,010 & 84.6 & 2.3 \\
\hline 09089500 & Mountain & 39.3311 & 107.5801 & 49 & 44 & 64.2 & 26.5 & 29.9 & 8,732 & 97.4 & 2.3 \\
\hline 09091100 & Northwest & 39.5316 & 107.7140 & 14 & NA & 63.4 & 23.4 & 20.2 & 7,243 & 40.7 & 2.1 \\
\hline 09091500 & Northwest & 39.6778 & 107.6984 & 8 & 15 & 34.9 & 33.5 & 26.1 & 8,550 & 91.5 & 2.5 \\
\hline 09092000 & Northwest & 39.6200 & 107.7634 & 20 & 19 & 137.1 & 32.4 & 22.9 & 7,972 & 65.5 & 2.2 \\
\hline 09092500 & Northwest & 39.4719 & 107.8326 & 30 & 30 & 7.9 & 29.0 & 29.4 & 9,406 & 94.3 & 2.2 \\
\hline 09093000 & Northwest & 39.5669 & 108.1109 & 24 & 23 & 141.5 & 34.6 & 21.2 & 8,134 & 91 & 2.1 \\
\hline 09095000 & Northwest & 39.4533 & 108.3170 & 23 & 22 & 323.1 & 42.2 & 20.2 & 7,624 & 64.8 & 2 \\
\hline 09096000 & Mountain & 39.2236 & 107.8020 & 13 & 12 & 22.8 & 20.4 & 31.2 & 9,365 & 100 & 2.2 \\
\hline 09096500 & Mountain & 39.2505 & 107.8406 & 37 & 59 & 80.4 & 17.4 & 34.3 & 9,743 & 99.5 & 2.4 \\
\hline 09096800 & Mountain & 39.2361 & 107.6339 & 15 & 15 & 49.8 & 17.5 & 30.3 & 9,351 & 100 & 2.4 \\
\hline 09097500 & Mountain & 39.2722 & 107.8506 & 59 & 59 & 143 & 20.6 & 26.7 & 8,622 & 92.4 & 2.3 \\
\hline 09097600 & Mountain & 39.3250 & 107.8423 & 12 & 12 & 9.3 & 36.9 & 30.9 & 9,589 & 100 & 2.2 \\
\hline 09104500 & Northwest & 39.0864 & 108.1267 & 24 & 20 & 5.3 & 21.5 & 34.1 & 9,862 & 100 & 2.4 \\
\hline 09107000 & Mountain & 38.8603 & 106.5667 & 24 & 25 & 127.6 & 27.3 & 26.6 & 10,924 & 100 & 2.3 \\
\hline 09110000 & Mountain & 38.6644 & 106.8453 & 20 & 97 & 477.1 & 30.9 & 23.9 & 10,647 & 100 & 2.2 \\
\hline 09110500 & Mountain & 38.8644 & 106.9098 & 12 & 11 & 89.2 & 40.7 & 38.2 & 10,883 & 100 & 2.4 \\
\hline 09111500 & Mountain & 38.8697 & 106.9695 & 25 & 24 & 68.9 & 36.8 & 33.6 & 10,336 & 100 & 2.3 \\
\hline 09112000 & Mountain & 38.8244 & 106.8528 & 12 & 14 & 32.9 & 37.8 & 32 & 10,808 & 100 & 2.4 \\
\hline 09112500 & Mountain & 38.6644 & 106.8481 & 76 & 85 & 289.3 & 34.2 & 31.7 & 10,272 & 100 & 2.3 \\
\hline 09113300 & Mountain & 38.7655 & 107.0584 & 12 & 12 & 47.7 & 32.2 & 31.9 & 10,202 & 100 & 2.3 \\
\hline 09113500 & Mountain & 38.7022 & 106.9984 & 26 & 25 & 119.3 & 28.6 & 27.3 & 9,894 & 100 & 2.1 \\
\hline 09114500 & Mountain & 38.5419 & 106.9498 & 78 & 81 & 1,010 & 29.8 & 25.6 & 10,210 & 100 & 2.2 \\
\hline 09115500 & Mountain & 38.4117 & 106.4228 & 55 & 56 & 148.3 & 31.2 & 21.4 & 10,237 & 100 & 2.2 \\
\hline 09117000 & Mountain & 38.4972 & 106.7261 & 14 & 14 & 425.9 & 26.3 & 18.3 & 9,606 & 100 & 2.1 \\
\hline 09118000 & Mountain & 38.5597 & 106.6364 & 24 & 24 & 106.9 & 38.3 & 23.4 & 10,619 & 100 & 2.2 \\
\hline 09119000 & Mountain & 38.5217 & 106.9409 & 68 & 70 & $1,059.9$ & 24.2 & 18.6 & 9,733 & 100 & 2.1 \\
\hline 09122000 & Mountain & 38.2914 & 107.1145 & 18 & 18 & 339.9 & 26.4 & 21.1 & 10,432 & 100 & 2.2 \\
\hline
\end{tabular}


Table 1. Drainage-basin and climatic characteristics at streamflow-gaging stations used for regional regression analysis.-Continued

[LATDEG, latitude in decimal degrees; LNGDEG, longitude in decimal degrees; YRSPK, years of record for peak streamflow; YRSANN, years of record for annual streamflow; $A$, drainage area, in square miles; $S$, mean watershed slope, in percent; $P$, mean annual precipitation, in inches; $E$, mean watershed elevation, in feet; $A_{7500}$, percentage of $A$ above 7,500 feet elevation plus $1 ;{ }_{6} P_{100}$, 6-hour, 100-year precipitation, in inches; NA, not applicable, station operated as peak-only or flood-hydrograph station]

\begin{tabular}{|c|c|c|c|c|c|c|c|c|c|c|c|}
\hline $\begin{array}{l}\text { Station } \\
\text { number }\end{array}$ & $\begin{array}{l}\text { Hydrologic } \\
\text { region }\end{array}$ & LATDEG & LNGDEG & YRSPK & YRSANN & $A$ & $S$ & $P$ & $E$ & $A_{7500}$ & ${ }_{6} P_{100}$ \\
\hline 09122500 & Mountain & 38.5608 & 107.3167 & 11 & 11 & 58.2 & 46.0 & 27.4 & 9,862 & 100 & 2.2 \\
\hline 09123500 & Mountain & 38.0250 & 107.3084 & 13 & 12 & 120.5 & 45.9 & 34.1 & 11,435 & 100 & 2.5 \\
\hline 09124500 & Mountain & 38.2989 & 107.2301 & 69 & 70 & 339.2 & 42.7 & 28.2 & 10,884 & 100 & 2.4 \\
\hline 09125000 & Mountain & 38.4878 & 107.4151 & 27 & 27 & 35 & 38.5 & 22.7 & 9,674 & 100 & 2.3 \\
\hline 09126000 & Mountain & 38.2572 & 107.5467 & 15 & 53 & 67 & 49.6 & 32.7 & 10,862 & 100 & 2.3 \\
\hline 09127500 & Mountain & 38.5519 & 107.5062 & 21 & 18 & 42.3 & 29.4 & 20.5 & 9,631 & 100 & 2.4 \\
\hline 09128500 & Northwest & 38.7278 & 107.5067 & 58 & 59 & 43.4 & 43.6 & 25.6 & 9,165 & 97.3 & 2.3 \\
\hline 09130500 & Northwest & 39.0133 & 107.3584 & 19 & 19 & 132.9 & 28.9 & 28.7 & 8,694 & 86.8 & 2.3 \\
\hline 09130600 & Mountain & 39.1308 & 107.5753 & 10 & 10 & 7.3 & 18.3 & 29.8 & 9,418 & 100 & 2.5 \\
\hline 09132500 & Northwest & 38.9258 & 107.4342 & 72 & 74 & 525.6 & 33.0 & 28.5 & 8,886 & 87.1 & 2.3 \\
\hline 09132900 & Mountain & 39.0322 & 107.6137 & 13 & 13 & 2.4 & 20.9 & 34 & 10,397 & 100 & 2.5 \\
\hline 09134500 & Northwest & 38.9264 & 107.7937 & 29 & 29 & 34.7 & 17.1 & 33.5 & 9,723 & 99.5 & 2.5 \\
\hline 09136200 & Northwest & 38.7830 & 107.8378 & 24 & 23 & $5,247.4$ & 28.9 & 22.0 & 9,444 & 91.2 & 2.1 \\
\hline 09137800 & Northwest & 38.9447 & 108.0281 & 12 & 12 & 10.8 & 17.4 & 32.8 & 9,663 & 99.5 & 2.5 \\
\hline 09139200 & Mountain & 38.9836 & 107.9720 & 12 & 12 & 12.2 & 18.6 & 37.7 & 10,153 & 100 & 2.5 \\
\hline 09140200 & Mountain & 38.9866 & 107.9437 & 12 & 12 & 5.6 & 16.1 & 38.3 & 9,969 & 100 & 2.5 \\
\hline 09141200 & Northwest & 38.9580 & 107.9187 & 12 & 12 & 10.5 & 17.3 & 33.9 & 9,381 & 98.1 & 2.4 \\
\hline 09143500 & Northwest & 38.9016 & 107.9212 & 90 & 82 & 39.1 & 16.8 & 35.7 & 9,464 & 94.2 & 2.5 \\
\hline 09144200 & Northwest & 38.7878 & 107.9953 & 23 & 22 & 197 & 17.6 & 24.4 & 8,034 & 58.3 & 2.2 \\
\hline 09146000 & Southwest & 38.0311 & 107.6751 & 16 & 16 & 75.2 & 58.4 & 38.1 & 11,267 & 100 & 2.4 \\
\hline 09146400 & Southwest & 38.0736 & 107.8512 & 15 & 15 & 14.1 & 38.5 & 30.9 & 10,215 & 100 & 2.3 \\
\hline 09146500 & Southwest & 38.0933 & 107.8137 & 16 & 16 & 16.5 & 50.3 & 35.2 & 10,939 & 100 & 2.4 \\
\hline 09146600 & Southwest & 38.1455 & 107.9198 & 12 & 12 & 8.2 & 11.8 & 25.8 & 9,053 & 100 & 2.2 \\
\hline 09147000 & Southwest & 38.1778 & 107.7584 & 49 & 49 & 97.2 & 28.1 & 26.3 & 9,163 & 91.4 & 2.2 \\
\hline 09147100 & Mountain & 38.1494 & 107.6448 & 18 & 18 & 45.6 & 55.6 & 33.7 & 10,721 & 100 & 2.4 \\
\hline 09149450 & Northwest & 38.5553 & 108.0459 & 13 & NA & 102.7 & 15.3 & 18.2 & 7,839 & 60.2 & 2 \\
\hline 09150500 & Northwest & 38.7350 & 108.1617 & 24 & 23 & 249.1 & 18.3 & 17.4 & 7,274 & 44.5 & 2 \\
\hline 09153400 & Northwest & 39.3086 & 108.9837 & 10 & 10 & 168.4 & 41.8 & 15.5 & 6,525 & 15.2 & 2 \\
\hline 09163700 & Southwest & 38.9639 & 109.3368 & 15 & NA & 90.1 & 15.5 & 9.9 & 4,980 & 0.2 & 1.8 \\
\hline 09165000 & Southwest & 37.6389 & 108.0604 & 53 & 54 & 105.6 & 38.2 & 36.1 & 10,631 & 100 & 2.7 \\
\hline 09166500 & Southwest & 37.4725 & 108.4976 & 92 & 96 & 505.2 & 32.3 & 31.2 & 9,694 & 97.6 & 2.7 \\
\hline 09168100 & Southwest & 37.8767 & 108.5831 & 29 & 29 & 147 & 27.6 & 21.5 & 7,932 & 65.4 & 2.3 \\
\hline 09168700 & Southwest & 38.0258 & 108.8148 & 12 & NA & 1.7 & 9.7 & 12.9 & 5,836 & 0 & 2 \\
\hline 09169500 & Southwest & 38.3103 & 108.8854 & 40 & 41 & $2,028.6$ & 22.7 & 21.9 & 7,862 & 54.2 & 2.3 \\
\hline 09172000 & Southwest & 37.9583 & 108.0059 & 18 & 18 & 33.4 & 31.2 & 32.1 & 10,041 & 100 & 2.5 \\
\hline 09172500 & Southwest & 38.0425 & 108.1323 & 70 & 70 & 309.5 & 37.6 & 30.3 & 9,944 & 99 & 2.3 \\
\hline 09174500 & Southwest & 38.2736 & 108.3629 & 10 & 9 & 38.4 & 16.1 & 19.7 & 7,645 & 54.7 & 2.1 \\
\hline 09175000 & Southwest & 37.9758 & 108.3279 & 12 & 18 & 53.7 & 16.9 & 26.2 & 8,687 & 100 & 2.4 \\
\hline 09175500 & Southwest & 38.2178 & 108.5665 & 53 & 53 & $1,068.6$ & 22.6 & 23.7 & 8,483 & 73.3 & 2.2 \\
\hline 09177500 & Southwest & 38.5189 & 109.1098 & 23 & NA & 15.4 & 16.0 & 30.3 & 8,986 & 100 & 2.4 \\
\hline 09181000 & Southwest & 38.7250 & 109.3451 & 13 & 5 & 20.5 & 44.7 & 13.8 & 5,637 & 0.8 & 1.9 \\
\hline 09182000 & Southwest & 38.5928 & 109.2657 & 24 & 23 & 7.9 & 41.1 & 31.8 & 9,468 & 98.4 & 2.4 \\
\hline
\end{tabular}


[LATDEG, latitude in decimal degrees; LNGDEG, longitude in decimal degrees; YRSPK, years of record for peak streamflow; YRSANN, years of record for annual streamflow; $A$, drainage area, in square miles; $S$, mean watershed slope, in percent; $P$, mean annual precipitation, in inches; $E$, mean watershed elevation, in feet; $A_{7500}$, percentage of $A$ above 7,500 feet elevation plus $1 ;{ }_{6} P_{100}, 6$-hour, 100-year precipitation, in inches; NA, not applicable, station operated as peak-only or flood-hydrograph station]

\begin{tabular}{|c|c|c|c|c|c|c|c|c|c|c|c|}
\hline $\begin{array}{l}\text { Station } \\
\text { number }\end{array}$ & $\begin{array}{c}\text { Hydrologic } \\
\text { region }\end{array}$ & LATDEG & LNGDEG & YRSPK & YRSANN & $A$ & $s$ & $\boldsymbol{P}$ & $E$ & $A_{7500}$ & ${ }_{6} \boldsymbol{P}_{100}$ \\
\hline 09182600 & Southwest & 38.9528 & 109.6590 & 15 & NA & 5.8 & 31.7 & 11.1 & 5,743 & 0 & 1.9 \\
\hline 09183000 & Southwest & 38.6128 & 109.5798 & 31 & 29 & 163 & 14.8 & 9.6 & 4,807 & 0 & 1.8 \\
\hline 09184000 & Southwest & 38.5622 & 109.5140 & 47 & 43 & 77.1 & 29.6 & 19.9 & 7,104 & 37.6 & 2.2 \\
\hline 09185200 & Southwest & 38.4000 & 109.4507 & 15 & NA & 15.2 & 25.1 & 15 & 6,669 & 14.1 & 2.1 \\
\hline 09185500 & Southwest & 38.2433 & 109.4401 & 22 & 21 & 357.9 & 9.7 & 14.2 & 6,586 & 1.8 & 2.1 \\
\hline 09187000 & Southwest & 38.0625 & 109.5743 & 17 & 8 & 114.3 & 32.8 & 17.2 & 7,338 & 45.1 & 2.4 \\
\hline 09216400 & Northwest & 41.5594 & 109.5112 & 16 & NA & 45.9 & 16.7 & 9.5 & 7,042 & 12.4 & 1.7 \\
\hline 09216537 & Northwest & 41.6394 & 108.1293 & 24 & NA & 34.4 & 7.0 & 7.9 & 7,011 & 0.1 & 1.7 \\
\hline 09216550 & Northwest & 41.6750 & 108.7368 & 21 & NA & 153 & 7.9 & 8.5 & 6,976 & 4.7 & 1.7 \\
\hline 09216600 & Northwest & 41.4569 & 108.9423 & 22 & NA & 8.1 & 15.1 & 9.2 & 6,982 & 3.3 & 1.7 \\
\hline 09216700 & Northwest & 41.4833 & 108.9673 & 18 & NA & 481.9 & 12.5 & 11.2 & 7,317 & 31 & 1.7 \\
\hline 09216900 & Northwest & 41.5327 & 109.3810 & 24 & NA & 1.3 & 34.2 & 8.8 & 6,827 & 0 & 1.7 \\
\hline 09224810 & Northwest & 41.4597 & 109.6229 & 17 & NA & 12.1 & 10.2 & 8.6 & 6,654 & 0 & 1.7 \\
\hline 09224820 & Northwest & 41.4250 & 109.6160 & 20 & NA & 3.6 & 11.4 & 8.6 & 6,566 & 0 & 1.7 \\
\hline 09224840 & Northwest & 41.4111 & 109.6021 & 17 & NA & 1.3 & 15.0 & 8.6 & 6,553 & 0 & 1.7 \\
\hline 09224980 & Northwest & 41.3736 & 109.6451 & 17 & NA & 414.9 & 7.6 & 9.9 & 6,840 & 12.8 & 1.7 \\
\hline 09225200 & Northwest & 41.1705 & 109.6101 & 20 & NA & 6.7 & 15.9 & 10.3 & 6,653 & 2.9 & 1.7 \\
\hline 09225300 & Northwest & 41.0611 & 109.6188 & 21 & NA & 11.9 & 12.2 & 10.6 & 6,578 & 0 & 1.7 \\
\hline 09229450 & Northwest & 41.0205 & 109.6801 & 10 & NA & 525.3 & 15.8 & 19.3 & 8,541 & 67.4 & 2.2 \\
\hline 09235600 & Northwest & 40.7680 & 109.3190 & 35 & 36 & 24.9 & 19.9 & 20.5 & 8,139 & 100 & 2.6 \\
\hline 09238500 & Northwest & 40.4080 & 106.7870 & 20 & 13 & 42.3 & 19.6 & 47.1 & 9,493 & 98.1 & 2.4 \\
\hline 09239500 & Northwest & 40.4836 & 106.8323 & 99 & 99 & 567.4 & 21.7 & 31.5 & 8,782 & 86.3 & 2.2 \\
\hline 09241000 & Mountain & 40.7175 & 106.9159 & 69 & 68 & 216.5 & 27.3 & 38.1 & 9,106 & 99.4 & 2.4 \\
\hline 09244100 & Northwest & 40.3341 & 107.1392 & 18 & 18 & 34.5 & 25.2 & 26.3 & 8,292 & 73.6 & 2.1 \\
\hline 09245000 & Northwest & 40.6697 & 107.2851 & 44 & 43 & 67.7 & 20.4 & 30.7 & 8,414 & 94.5 & 2.2 \\
\hline 09245500 & Northwest & 40.6805 & 107.2873 & 15 & 15 & 21.4 & 22.7 & 31.4 & 8,461 & 89 & 2.2 \\
\hline 09249000 & Northwest & 40.3125 & 107.3201 & 19 & 18 & 144.1 & 23.8 & 33.7 & 9,063 & 92.3 & 2.3 \\
\hline 09250000 & Northwest & 40.1936 & 107.7323 & 34 & NA & 63.3 & 21.3 & 24.3 & 7,906 & 62.9 & 2.2 \\
\hline 09251500 & Northwest & 40.9905 & 107.0442 & 10 & 10 & 115.4 & 21.0 & 33.5 & 8,707 & 97.8 & 2.3 \\
\hline 09251800 & Northwest & 41.0425 & 106.9572 & 10 & 9 & 13 & 26.8 & 47 & 9,458 & 100 & 2.3 \\
\hline 09253000 & Northwest & 40.9994 & 107.1434 & 60 & 60 & 252.7 & 22.4 & 32.8 & 8,546 & 91.6 & 2.3 \\
\hline 09253400 & Northwest & 41.1322 & 107.0692 & 12 & NA & 12.7 & 24.1 & 48.6 & 9,573 & 100 & 2.3 \\
\hline 09255000 & Northwest & 40.9825 & 107.3828 & 76 & 76 & 149.8 & 19.4 & 30.5 & 8,390 & 85.6 & 2.2 \\
\hline 09256000 & Northwest & 41.0978 & 107.3819 & 38 & 36 & 332.4 & 15.3 & 26.6 & 7,837 & 74.8 & 2.1 \\
\hline 09257000 & Northwest & 41.0283 & 107.5492 & 59 & 46 & $1,010.9$ & 18.6 & 27.8 & 8,001 & 71.1 & 2.2 \\
\hline 09257500 & Northwest & 40.8766 & 107.4645 & 10 & NA & 8.4 & 18.9 & 38.1 & 9,011 & 100 & 2.2 \\
\hline 09258000 & Northwest & 40.9155 & 107.5217 & 39 & 39 & 24.8 & 18.7 & 28.2 & 8,066 & 70 & 2.1 \\
\hline 09258200 & Northwest & 41.3400 & 107.6712 & 12 & NA & 51.2 & 7.4 & 13.1 & 6,927 & 3.2 & 1.8 \\
\hline 09258900 & Northwest & 41.1319 & 107.6465 & 14 & NA & 920.5 & 8.7 & 13 & 6,988 & 11.7 & 1.8 \\
\hline 09259500 & Northwest & 40.8405 & 107.5151 & 11 & NA & 8.5 & 17.6 & 31.4 & 8,405 & 86.4 & 2.1 \\
\hline 09260000 & Northwest & 40.5486 & 108.4243 & 83 & 86 & $4,035.8$ & 11.7 & 16.6 & 7,063 & 21.8 & 1.9 \\
\hline 09263700 & Northwest & 40.3000 & 109.1340 & 15 & NA & 63.1 & 19.2 & 12.6 & 6,468 & 12 & 1.9 \\
\hline
\end{tabular}


Table 1. Drainage-basin and climatic characteristics at streamflow-gaging stations used for regional regression analysis.-Continued

[LATDEG, latitude in decimal degrees; LNGDEG, longitude in decimal degrees; YRSPK, years of record for peak streamflow; YRSANN, years of record for annual streamflow; $A$, drainage area, in square miles; $S$, mean watershed slope, in percent; $P$, mean annual precipitation, in inches; $E$, mean watershed elevation, in feet; $A_{7500}$, percentage of $A$ above 7,500 feet elevation plus $1 ;{ }_{6} P_{100}$, 6-hour, 100-year precipitation, in inches; NA, not applicable, station operated as peak-only or flood-hydrograph station]

\begin{tabular}{|c|c|c|c|c|c|c|c|c|c|c|c|}
\hline $\begin{array}{l}\text { Station } \\
\text { number }\end{array}$ & $\begin{array}{l}\text { Hydrologic } \\
\text { region }\end{array}$ & LATDEG & LNGDEG & YRSPK & YRSANN & $A$ & $S$ & $P$ & $E$ & $A_{7500}$ & ${ }_{6} \boldsymbol{P}_{100}$ \\
\hline 09263800 & Northwest & 40.3166 & 109.2173 & 14 & NA & 9.4 & 10.1 & 9.2 & 5,379 & 0 & 1.8 \\
\hline 09264000 & Northwest & 40.7333 & 109.6785 & 11 & 11 & 26.6 & 10.1 & 29.2 & 9,929 & 100 & 2.6 \\
\hline 09264500 & Northwest & 40.7333 & 109.7035 & 12 & 12 & 19.9 & 18.2 & 32.2 & 10,480 & 100 & 2.7 \\
\hline 09266500 & Northwest & 40.5775 & 109.6221 & 94 & 92 & 101.5 & 20.7 & 27.4 & 9,452 & 96.7 & 2.5 \\
\hline 09268000 & Northwest & 40.6263 & 109.8201 & 37 & 36 & 44.6 & 20.8 & 31.8 & 10,353 & 100 & 2.7 \\
\hline 09268500 & Northwest & 40.6427 & 109.8110 & 44 & 43 & 8.8 & 24.5 & 30.9 & 10,138 & 100 & 2.7 \\
\hline 09268900 & Northwest & 40.6594 & 109.7510 & 29 & 29 & 7.5 & 18.0 & 30.5 & 10,030 & 100 & 2.6 \\
\hline 09269000 & Northwest & 40.6500 & 109.7618 & 18 & 17 & 10.4 & 19.1 & 29.5 & 9,832 & 100 & 2.6 \\
\hline 09270500 & Northwest & 40.5263 & 109.6057 & 35 & 35 & 116.2 & 24.7 & 26.7 & 9,279 & 86.6 & 2.5 \\
\hline 09271800 & Northwest & 40.4166 & 109.7507 & 15 & NA & 4.3 & 20.8 & 12.7 & 6,542 & 12.9 & 1.9 \\
\hline 09300500 & Northwest & 40.3019 & 109.8532 & 28 & 22 & 544.6 & 21.6 & 23.1 & 8,833 & 66.2 & 2.4 \\
\hline 09302450 & Mountain & 40.0503 & 107.4689 & 25 & 25 & 21.6 & 21.8 & 30.3 & 8,971 & 100 & 2.4 \\
\hline 09302500 & Mountain & 40.0383 & 107.4881 & 12 & 12 & 59.9 & 25.8 & 37.8 & 9,814 & 100 & 2.5 \\
\hline 09303000 & Northwest & 39.9875 & 107.6145 & 50 & 56 & 259.4 & 25.2 & 35.5 & 9,551 & 98 & 2.4 \\
\hline 09303300 & Mountain & 39.8433 & 107.3348 & 20 & 20 & 52.2 & 21.5 & 42.1 & 10,585 & 100 & 2.7 \\
\hline 09303320 & Mountain & 39.8428 & 107.3367 & 14 & 14 & 7.4 & 14.2 & 41.8 & 10,667 & 100 & 2.8 \\
\hline 09304000 & Northwest & 39.9744 & 107.6253 & 46 & 47 & 176.5 & 27.2 & 36.9 & 9,852 & 97.5 & 2.5 \\
\hline 09304500 & Northwest & 40.0336 & 107.8623 & 98 & 103 & 760.3 & 24.9 & 30.9 & 8,938 & 84 & 2.4 \\
\hline 09306007 & Northwest & 39.8261 & 108.1831 & 25 & 24 & 177.3 & 32.7 & 19.9 & 7,634 & 57.6 & 2.1 \\
\hline 09306036 & Northwest & 39.8250 & 108.1993 & 12 & 11 & 3.6 & 14.3 & 16.7 & 6,933 & 0 & 2 \\
\hline 09306039 & Northwest & 39.8266 & 108.2076 & 11 & 11 & 1.2 & 13.8 & 16.4 & 6,744 & 0 & 2 \\
\hline 09306042 & Northwest & 39.8336 & 108.2206 & 18 & 16 & 1.1 & 15.4 & 16.5 & 6,671 & 0 & 2 \\
\hline 09306052 & Northwest & 39.8141 & 108.2437 & 11 & 9 & 7.9 & 19.5 & 17.3 & 7,213 & 23.8 & 2 \\
\hline 09306058 & Northwest & 39.8372 & 108.2443 & 11 & 11 & 48.3 & 28.8 & 18.1 & 7,473 & 48.9 & 2 \\
\hline 09306200 & Northwest & 39.9211 & 108.2976 & 41 & 42 & 505.8 & 27.9 & 18.7 & 7,419 & 45.1 & 2.1 \\
\hline 09306240 & Northwest & 39.8883 & 108.5284 & 11 & 11 & 9.2 & 28.3 & 20.5 & 7,876 & 78 & 2 \\
\hline 09306242 & Northwest & 39.9203 & 108.4729 & 33 & 33 & 31.7 & 24.2 & 19.2 & 7,542 & 49.6 & 2 \\
\hline 09306255 & Northwest & 40.1686 & 108.4012 & 28 & 29 & 262.5 & 19.5 & 16.7 & 6,878 & 17.7 & 1.9 \\
\hline 09306800 & Northwest & 39.7533 & 109.3548 & 19 & 19 & 323.3 & 29.2 & 15.9 & 7,157 & 32.3 & 2 \\
\hline 09307500 & Northwest & 39.5664 & 109.5874 & 27 & 27 & 300 & 37.6 & 17.5 & 7,772 & 63.2 & 2.2 \\
\hline 09308000 & Northwest & 39.9389 & 109.6485 & 26 & 17 & 902.7 & 28.5 & 15.1 & 7,112 & 35.5 & 2 \\
\hline 09328900 & Southwest & 38.9422 & 109.8212 & 10 & NA & 22.9 & 37.8 & 12.1 & 6,110 & 0.4 & 2 \\
\hline 09339900 & Southwest & 37.3897 & 106.8412 & 45 & 45 & 65.4 & 44.4 & 43 & 10,235 & 100 & 3.1 \\
\hline 09340000 & Southwest & 37.3695 & 106.8923 & 46 & 45 & 90.8 & 45.7 & 41.5 & 10,071 & 100 & 3 \\
\hline 09340500 & Southwest & 37.4856 & 106.9303 & 17 & 16 & 39.9 & 46.9 & 51 & 11,081 & 100 & 3.1 \\
\hline 09341500 & Southwest & 37.3920 & 106.9073 & 29 & 28 & 85.6 & 43.3 & 45 & 10,380 & 100 & 3.1 \\
\hline 09342000 & Southwest & 37.3695 & 106.9403 & 13 & 12 & 24 & 40.2 & 39.9 & 9,890 & 100 & 3.1 \\
\hline 09342500 & Southwest & 37.2667 & 107.0078 & 75 & 72 & 280.8 & 38.9 & 38.8 & 9,738 & 96.1 & 3 \\
\hline 09343000 & Southwest & 37.2128 & 106.7945 & 37 & 36 & 57.9 & 46.7 & 39.2 & 10,026 & 100 & 3.1 \\
\hline 09343500 & Southwest & 37.1936 & 106.9053 & 18 & 17 & 23.1 & 32.5 & 33.1 & 9,264 & 99.1 & 2.8 \\
\hline 09344000 & Southwest & 37.0853 & 106.6895 & 41 & 59 & 69.2 & 43.3 & 40.1 & 10,255 & 100 & 3 \\
\hline 09345200 & Southwest & 37.0756 & 106.8111 & 8 & 25 & 13.4 & 26.0 & 33.2 & 9,608 & 100 & 2.9 \\
\hline
\end{tabular}


[LATDEG, latitude in decimal degrees; LNGDEG, longitude in decimal degrees; YRSPK, years of record for peak streamflow; YRSANN, years of record for annual streamflow; $A$, drainage area, in square miles; $S$, mean watershed slope, in percent; $P$, mean annual precipitation, in inches; $E$, mean watershed elevation, in feet; $A_{7500}$, percentage of $A$ above 7,500 feet elevation plus $1 ;{ }_{6} P_{100}, 6$-hour, 100-year precipitation, in inches; NA, not applicable, station operated as peak-only or flood-hydrograph station]

\begin{tabular}{|c|c|c|c|c|c|c|c|c|c|c|c|}
\hline $\begin{array}{l}\text { Station } \\
\text { number }\end{array}$ & $\begin{array}{c}\text { Hydrologic } \\
\text { region }\end{array}$ & LATDEG & LNGDEG & YRSPK & YRSANN & $\boldsymbol{A}$ & $S$ & $\boldsymbol{P}$ & $E$ & $A_{7500}$ & ${ }_{6} \boldsymbol{P}_{100}$ \\
\hline 09346000 & Southwest & 37.0028 & 106.9075 & 36 & 71 & 176 & 32.3 & 31.4 & 9,180 & 95.4 & 2.8 \\
\hline 09346200 & Southwest & 36.9333 & 107.0006 & 39 & NA & 129.5 & 18.1 & 20.9 & 7,637 & 67.1 & 2.5 \\
\hline 09347500 & Southwest & 37.4286 & 107.1934 & 14 & 13 & 82.6 & 41.9 & 37.8 & 10,137 & 100 & 3 \\
\hline 09349800 & Southwest & 37.0883 & 107.3978 & 44 & 45 & 624.9 & 29.3 & 27.2 & 8,564 & 78.5 & 2.7 \\
\hline 09350800 & Southwest & 36.7231 & 107.2797 & 49 & NA & 60.2 & 18.2 & 18 & 7,394 & 32.5 & 2.5 \\
\hline 09352900 & Southwest & 37.4775 & 107.5437 & 43 & 45 & 72.5 & 57.9 & 39.5 & 11,350 & 100 & 3.5 \\
\hline 09355000 & Southwest & 37.0111 & 107.5970 & 56 & 56 & 58.3 & 16.7 & 17.4 & 6,940 & 20.6 & 2.1 \\
\hline 09357200 & Southwest & 36.4667 & 107.9173 & 35 & NA & 0.2 & 14.4 & 10.5 & 6,934 & 0 & 2.1 \\
\hline 09357230 & Southwest & 36.5900 & 108.1848 & 21 & NA & 0.3 & 9.9 & 9.6 & 5,958 & 0 & 2 \\
\hline 09357500 & Southwest & 37.8331 & 107.5995 & 47 & 47 & 57.6 & 54.4 & 44.7 & 11,936 & 100 & 2.7 \\
\hline 09358550 & Southwest & 37.8197 & 107.6637 & 14 & 15 & 20.1 & 54.5 & 40.5 & 11,445 & 100 & 2.7 \\
\hline 09359000 & Southwest & 37.7975 & 107.6953 & 14 & 13 & 44.4 & 51.9 & 41.2 & 11,571 & 100 & 2.8 \\
\hline 09361000 & Southwest & 37.4219 & 107.8451 & 50 & 48 & 168.4 & 46.7 & 33.7 & 9,592 & 98.7 & 2.8 \\
\hline 09361400 & Southwest & 37.3342 & 107.9095 & 20 & 6 & 25.5 & 53.9 & 34.1 & 9,413 & 97.5 & 3.1 \\
\hline 09361500 & Southwest & 37.2792 & 107.8803 & 83 & 95 & 709.6 & 46.6 & 35.9 & 10,146 & 93.5 & 2.9 \\
\hline 09362000 & Southwest & 37.6039 & 107.8937 & 22 & 22 & 63.2 & 33.0 & 24.8 & 8,174 & 68.7 & 2.7 \\
\hline 09363000 & Southwest & 37.3253 & 107.7490 & 45 & 42 & 97.4 & 34.1 & 34.4 & 9,999 & 99.4 & 3.5 \\
\hline 09363100 & Southwest & 37.1397 & 107.7534 & 23 & 23 & 17.8 & 6.8 & 16.9 & 6,762 & 0 & 2.2 \\
\hline 09365500 & Southwest & 37.2897 & 108.0406 & 92 & 90 & 33.4 & 49.2 & 39.8 & 10,228 & 100 & 3 \\
\hline 09366000 & Southwest & 37.1189 & 108.1987 & 23 & 22 & 75.6 & 23.3 & 21.4 & 7,835 & 59.6 & 2.4 \\
\hline 09366500 & Southwest & 36.9997 & 108.1887 & 86 & 87 & 309.3 & 18.0 & 20.3 & 7,605 & 39.5 & 2.4 \\
\hline 09367400 & Southwest & 36.7861 & 108.2259 & 27 & 4 & 1.1 & 15.3 & 9.8 & 5,650 & 0 & 2 \\
\hline 09367530 & Southwest & 36.7333 & 108.3006 & 35 & NA & 3 & 7.6 & 9.2 & 5,503 & 0 & 1.9 \\
\hline 09367550 & Southwest & 36.7667 & 108.3701 & 21 & NA & 4.6 & 6.6 & 9 & 5,468 & 0 & 1.9 \\
\hline 09367561 & Southwest & 36.7733 & 108.4412 & 16 & 15 & 136.3 & 13.0 & 10.7 & 5,776 & 0 & 2 \\
\hline 09367950 & Southwest & 36.7244 & 108.5915 & 30 & 18 & $4,390.7$ & 7.3 & 9.9 & 6,328 & 4.5 & 2.2 \\
\hline 09367980 & Southwest & 36.7706 & 108.7262 & 17 & NA & 21.7 & 3.5 & 7.9 & 5,227 & 0 & 2 \\
\hline 09368020 & Southwest & 36.9258 & 108.7250 & 21 & NA & 2.1 & 6.1 & 8.8 & 5,334 & 0 & 2 \\
\hline 09368500 & Southwest & 37.3817 & 108.2581 & 16 & 15 & 39.5 & 26.3 & 33.4 & 9,705 & 99.9 & 2.8 \\
\hline 09369000 & Southwest & 37.3703 & 108.2315 & 15 & 14 & 12.1 & 43.0 & 33.1 & 9,690 & 100 & 2.7 \\
\hline 09369500 & Southwest & 37.3739 & 108.2306 & 15 & 13 & 12.1 & 25.3 & 31 & 9,388 & 100 & 2.7 \\
\hline 09371000 & Southwest & 37.0275 & 108.7415 & 72 & 78 & 526.7 & 26.4 & 19 & 7,216 & 28.8 & 2.3 \\
\hline 09371300 & Southwest & 37.3475 & 108.4829 & 11 & NA & 4.5 & 22.6 & 17.3 & 6,856 & 7.8 & 2.3 \\
\hline 09372000 & Southwest & 37.3242 & 109.0157 & 56 & 56 & 347.5 & 15.5 & 14.9 & 6,407 & 3.4 & 2.2 \\
\hline 09378170 & Southwest & 37.8467 & 109.3696 & 21 & 22 & 8.4 & 27.7 & 26.8 & 8,621 & 91.4 & 2.7 \\
\hline 09378630 & Southwest & 37.7556 & 109.4765 & 41 & 42 & 3.8 & 35.7 & 26.3 & 8,680 & 93.6 & 2.7 \\
\hline 09378650 & Southwest & 37.6808 & 109.4626 & 17 & 18 & 37.4 & 26.1 & 22.5 & 7,917 & 58.4 & 2.6 \\
\hline 09378700 & Southwest & 37.5606 & 109.5787 & 29 & 23 & 205 & 28.9 & 15.4 & 6,807 & 21.7 & 2.4 \\
\hline 09379000 & Southwest & 37.2661 & 109.6757 & 10 & 9 & 278.9 & 22.8 & 10.6 & 5,861 & 5.5 & 2.2 \\
\hline 09379030 & Southwest & 36.3333 & 109.6243 & 15 & NA & 76.9 & 12.3 & 8.3 & 5,905 & 0 & 2.2 \\
\hline 09379060 & Southwest & 36.4694 & 109.4062 & 14 & NA & 1.3 & 3.3 & 8.9 & 5,826 & 0 & 2.1 \\
\hline 09379300 & Southwest & 37.2167 & 109.8173 & 15 & NA & 64.4 & 21.1 & 8.6 & 5,383 & 0 & 2 \\
\hline
\end{tabular}




\section{Selection of Stations for Other Streamflow Statistics}

Many USGS streamflow-gaging stations in the study area operate as peak-only or flood-hydrograph stations and as such do not record daily-mean streamflow data. However, the remaining streamflow statistics or "other streamflow statistics" (nonannual peak) considered in this report are computed from continuous daily-mean streamflow. Hence, a subset of the 422 stations needed to be identified for the purposes of this study.

Stations used for development of equations for estimating the annual- and monthly-mean flow characteristics, the minimum and maximum streamflow characteristics, and the flow-duration curve are a subset of the 422 stations described in the previous section. A total of 288 sites with a combined record of 9,877 years of annual mean streamflow were selected. These stations are identified in table 1 as those with an applicable count of annual streamflow values. The actual number of stations used in the other streamflow-statistic equations is slightly reduced to counts between 210 and 280 because of factors such as the presence of zero-flow values or periods of partial streamflow record.

Finally, the authors concluded that the number of stations in the Plains hydrologic region was inadequate for regression-equation development for any of the streamflow statistics other than peak streamflow. Additionally, the presence of substantial record of zero flows at many stations throughout the region, which is attributed in part to the lack of abundant precipitation and sandy soils, makes direct application of conventional regional-regression techniques, such as shown in this report, difficult and any resulting equations of questionable applicability.

\section{Computation of Streamflow Statistics and Basin and Climatic Characteristics}

The methods used for computing the streamflow statistics and basin and climatic characteristics used in the development of the regression equations are described in this section. The resulting streamflow statistics and basin and climate characteristics are used as the respective response and predictor variables in the regression analysis.

\section{Peak-Streamflow Frequency}

The annual peak-streamflow data by station were used to estimate station-specific peak-streamflow magnitude by recurrence intervals, such as the 100-year peak streamflow. Peak streamflow estimates by station and $T$-year recurrence interval provide the basis for computing regional peakstreamflow regression equations.
The station-specific peak-streamflow values used for this report were computed using the computer program PeakFQ (Flynn and others, 2006) for all annual-peak streamflows representative of natural-flow conditions. PeakFQ is based on the guidelines for estimating peak-streamflow frequency that are described by the Interagency Advisory Committee on Water Data (1982). The guidelines are commonly referred to by practitioners as "Bulletin 17B."

The Bulletin 17B guidelines fit a Pearson Type III distribution to the product moments of the logarithms of annual peak streamflow. The product moments are mean, standard deviation, and skew. To compensate for effects of short record at a station, station skew is combined or weighted with a generalized value that is derived from a generalized skew map, which is included in Bulletin 17B. The program also provides for conditional probability adjustments for sites with zero-flow values for the annual peak. All other default settings of PeakFQ were used, and the program was operated in a batch or automated mode by using default settings to estimate station-specific, peak streamflow by recurrence intervals for this study. These station-specific, peak-streamflow values are considered the results of intermediate computations and are not reported here.

\section{Annual- and Monthly-Mean Streamflow and Flow-Duration Curves}

Daily-mean streamflow data for the 288 stations were used to compute the annual- and monthly-mean streamflows and the station-specific flow-duration curves. Not all 288 stations could be used, however, because of zero-flow conditions. Four sites have annual-mean streamflow values of zero, and various stations have one or more monthly-mean streamflows of zero. Station-specific annual- and monthlymean streamflows and flow-duration curve values provide the basis for developing regional equations for estimation of these streamflow statistics. Annual-mean streamflows are computed as the arithmetic mean of all annual-mean streamflows representative of natural-flow conditions. Monthlymean streamflows are computed as the arithmetic mean of all monthly-mean streamflows representative of natural-flow conditions. The flow-duration values are computed by sorting or ranking the daily-mean streamflow data and linearly interpolating to the five exceedance percentiles of interest $(10,25$, 50,75 , and 90).

\section{Minimum and Maximum 7-Day Streamflow}

Minimum- and maximum 7-day, $T$-year streamflows are computed from analysis of the 7-day minimum and maximum daily-mean streamflow for each year of record representative of natural-flow conditions. The 7-day, $T$-year statistics are the minimum or maximum consecutive 7-day mean streamflow 
expected to occur once every $T$-years or that has an exceedance probability of $1 / T$, and these statistics provide the basis for developing regional equations for estimation of minimum and maximum 7-day streamflow.

Minimum- and maximum-streamflow frequency statistics were estimated using the methods described by Riggs (1972) implemented by the computer program SWSTAT (Lumb and others, 1990). The use of SWSTAT in the context of this report requires the explicit assumption that the log-Pearson Type III distribution is simultaneously suitable for low- and high-flow frequency analysis in Colorado. The general approach implemented by SWSTAT includes the following: Annual series of the minimum or maximum 7-day streamflows by station are computed by a "sliding window," and the product moments of mean, standard deviation, and skew of the logarithms of the streamflow values are computed. Finally, a log-Pearson Type III distribution is fit to the product moments, and the quantiles for the 2-year, 10-year, and 50-year recurrence intervals are extracted to produce the station-specific minimum and maximum 7-day, $T$-year streamflow statistics.

\section{Basin and Climatic Characteristics}

Based on the results of previous regional streamflow studies conducted in Colorado and neighboring States (Kircher and others, 1985; Thomas and others, 1997; Vaill, 1999; Hortness and Berenbrock, 2001; Berenbrock, 2002; Miller, 2003; Hortness, 2006; Waltemeyer, 2006; and Kenney and others, 2007) and on the availability of readily accessible data, a total of 17 characteristics (11 basin and 6 climatic characteristics) that are listed in table 2 were evaluated as candidate predictor variables in the regression analysis. For each of the 422 stations utilized in this study for the development of the regression equations, values for all 17 characteristics were calculated using various techniques involving geographic information system technology and multiple data sources that are listed in table 3.

Exploratory graphical and statistical analysis of relations between the basin and climatic characteristics was conducted to narrow the list of candidate characteristics into those that are appropriate or applicable variables for regression analysis. The graphical and statistical analysis considered strength of correlation or concordance, linearity, and crosscorrelation between variables. For example, mean annual precipitation and mean watershed elevation are strongly related in Colorado; so, to a degree, having one is equivalent to having the other. The decision process also is influenced by the number of stations (degrees of freedom) by region. The graphical and statistical analysis resulted in selection of the six predictor variables of drainage area $(A)$ in square miles; mean watershed elevation $(E)$ in feet; mean watershed slope $(S)$ in percent; percentage of drainage area above 7,500 feet of elevation $\left(A_{7500}\right)$; mean annual precipitation $(P)$ in inches; and 6-hour, 100-year precipitation $\left({ }_{6} P_{100}\right)$ in inches. Only these six variables are considered for the remainder of this report.

Table 2. Basin and climatic characteristics evaluated for use in regional regression equations.

[NED, National Elevation Dataset; PRISM, Parameter-Elevation Regressions on Independent Slopes Model; km, kilometer; NOAA, National Oceanic and Atmospheric Administration; DAYMET, Daymet project]

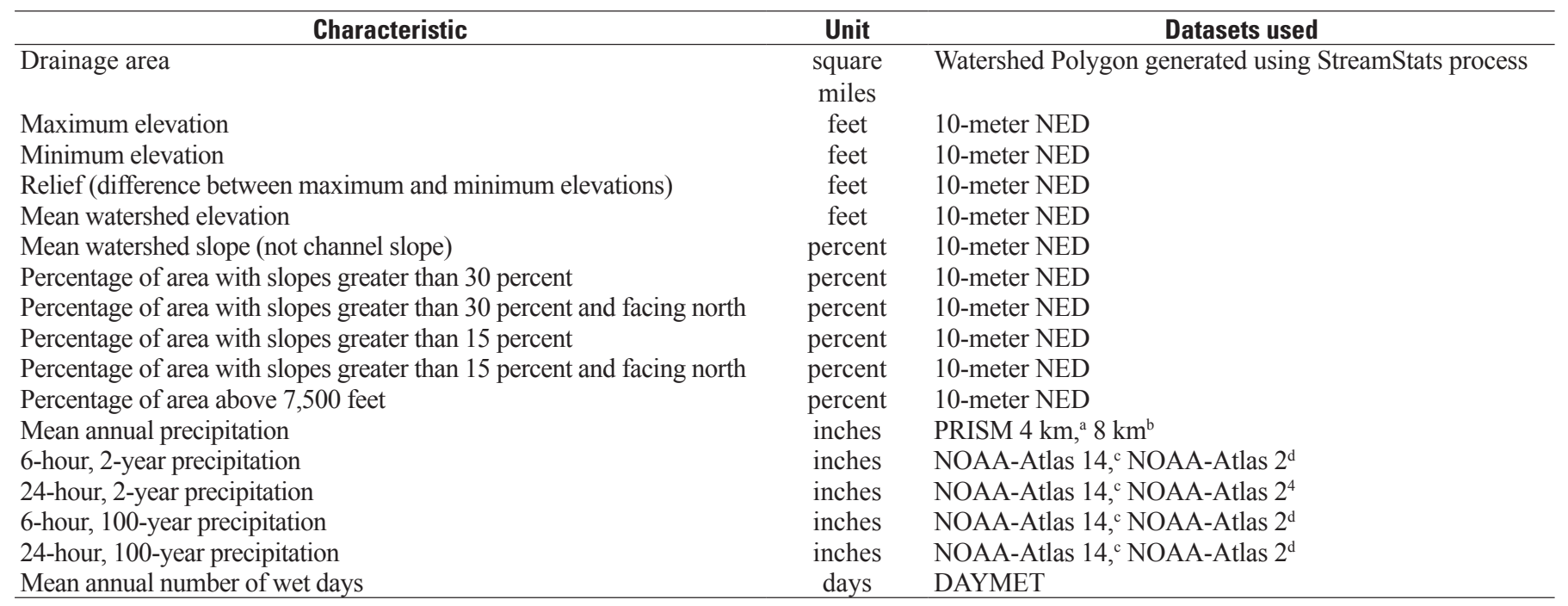

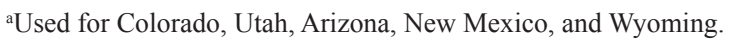

${ }^{b}$ Used for Nebraska, Kansas, Oklahoma, and Texas.

'Used for Utah, Arizona, and New Mexico.

${ }^{\mathrm{d}}$ Used for Colorado, Wyoming, Nebraska, Kansas, Oklahoma, and Texas. 
Table 3. Sources of data used to compute basin and climatic characteristics.

\begin{tabular}{cc}
\hline \multicolumn{1}{c}{ Dataset name } & Source description \\
\hline Parameter-elevation Regressions on Independent & Daly, Christopher, Nielson, R.P., and Phillips, D.L., 1994, A statistical-topographic \\
Slopes Model climate mapping system (PRISM) & model for mapping climatological precipitation over mountainous terrain: Journal \\
Total Precipitation (30-year average, 1971-2000) & of Applied Meteorology, v. 33, no. 2, p. 140-158, accessed August 15, 2007, \\
& at http://www.prism.oregonstate.edu/products/ \\
& Daymet Project, Daily surface weather and climatological summaries at 1-kilometer \\
Daily surface weather and climatological summaries & resolution over the conterminous United States, accessed August 15, 2007, at \\
& http://www.daymet.org \\
& Bonnin, G., Martin, D., Lin, B., Parzybok, T., Yekta, M., and Riley, D., 2006, Precipita- \\
Precipitation frequency atlas for the Western & tion frequency Atlas of the United States: National Weather Service, National Oceanic \\
United States (National Oceanic and Atmospheric & and Atmospheric Administration Atlas 14, Volume 1, version 4.0, accessed August 16, \\
Administration Atlas 14) & 2007, at http://hdsc.nws.noaa.gov/hdsc/pfds/docs/NA14Voll.pdf \\
& Miller, J.F., Frederick, R.H., and Tracy, R. J., 1973, Precipitation frequency \\
Precipitation frequency atlas for the Western & Atlas of the United States: National Weather Service, National Oceanic and \\
United States (National Oceanic and Atmospheric & Atmospheric Administration Atlas 2, Volume III, accessed August 16, 2007, \\
Administration Atlas 2) & at http://www.nws.noaa.gov/oh/hdsc/noaaatlas2.htm \\
& U.S. Geological Survey, 2007, National elevation dataset: accessed August 13, 2007, \\
National Elevation Dataset (NED) 10-meter resolution & at http://ned.usgs.gov/ \\
\hline
\end{tabular}

\section{Computation of Regional Regression Equations}

The computation of the regional regression equations is described in this section. Multilinear regression was used to define statistical relations between each of the streamflow statistics and two or more basin and climatic characteristics. A description of the principles of regional regression is beyond the scope of this report. Descriptions of the principles are available in references such as Helsel and Hirsch (2002) or Montgomery and others (2001). For the current (2009) investigation, logarithmic transformations (base-10) were made on all streamflow statistics and basin and climatic characteristics prior to the development of the regression equations. The data were logarithmically transformed in order to increase linearity between the regression variables and to acquire a constant variance of the regression residuals. For the $A_{7500}$ variable, which is presented as a percentage from 0 to 100 , a value of 1 percent was added before logarithmic transformation to avoid null values associated with the inability to take a logarithm of zero.

For the current (2009) investigation, two forms of related regression techniques were used - generalized least-squares (GLS) and weighted least-squares (WLS) regression analysis. GLS regression was used exclusively to compute equations for peak-streamflow and 7-day minimum and maximum streamflow estimation. WLS was used exclusively to compute equations for annual- and monthly-mean streamflow and flowduration curve estimation.

\section{Generalized Least-Squares Regression}

The regionalized regression equations for computing the peak-, minimum-, and maximum-streamflow equations were developed using the GLS method described by Stedinger and Tasker (1985) and Tasker and Stedinger (1989). For the regional streamflow-frequency regression analysis reported here, GLS regression was preferred over WLS regression because GLS is an extension of WLS that accounts for cross correlation between response variables. WLS regression only accounts for differences in station record length and uncertainty in the station-specific streamflow statistics and assumes independence between response variables. A summary of variables, units, and regression diagnostics reported for the GLS and WLS regression equations is shown in figure 2 .

The regression equations from GLS regression for estimation of peak, minimum, and maximum streamflow are listed by region in the left-hand page of figures $3-7$. The figures identify the streamflow statistic, the regression method, the number of respective stations used to develop the equations, numerical values of regression diagnostics, and a "check mark" that signifies the general reliability of the equation based on inspection of residual plots. For the 7-day minimum streamflow equations the station count decreases as the recurrence interval increases because of increases in the number of zero values in the station-specific estimates of the 7-day minimum streamflow.

Figures 3-7 provide appropriate GLS-regression diagnostics for each equation. These are the standard error of prediction, the pseudo R-squared statistic, and the standard model error. Readers are directed to Stedinger and Tasker (1985) and Tasker and Stedinger (1989) for details. Each equation is statistically significant with a p-value less than 0.01 . The ranges of the predictor variables also are listed in the figures. These ranges can be used to assess equation applicability. The applicability of the equations for ungaged sites with basin and climate characteristics outside the range of the predictor variables is questionable. 


\begin{tabular}{|c|c|}
\hline \multicolumn{2}{|r|}{ Regression Equation Response Variables } \\
\hline Variable & Variable definition \\
\hline$Q_{\mathrm{T}}$ & $T$-year peak streamflow, in cubic feet per second \\
\hline${ }_{7} Q_{\mathrm{T}}^{\min }$ & 7-day, $T$-year minimum streamflow, in cubic feet per second \\
\hline${ }_{7} Q_{\mathrm{T}}^{\max }$ & 7-day, $T$-year maximum streamflow, in cubic feet per second \\
\hline$Q_{\text {ann }}$ & Annual-mean streamflow, in cubic feet per second \\
\hline$Q_{\text {month }}$ & Monthly-mean streamflow by abbreviation, in cubic feet per second \\
\hline$Q_{\text {\#th }}$ & Daily-mean streamflow as exceedance probability as percent, in cubic feet per second \\
\hline \multicolumn{2}{|r|}{ Regression Equation Predictor Variables } \\
\hline Variable & Variable definition \\
\hline$A$ & Drainage area, in square miles \\
\hline$A_{7500}$ & Percentage of $A$ above 7,500 feet of elevation plus 1 \\
\hline$E$ & Mean elevation of watershed, in feet \\
\hline$P$ & Mean annual precipitation, in inches \\
\hline${ }_{6} P_{100}$ & 6-hour, 100-year precipitation, in inches \\
\hline$S$ & Mean watershed slope, in percent \\
\hline \multicolumn{2}{|r|}{ Regression Equation Diagnostics for GLS and WLS Regression Equations } \\
\hline Diagnostic & Diagnostic Definition \\
\hline SEP & Standard error of prediction (WLS and GLS), in percent \\
\hline $\operatorname{adj} R^{2}$ & $100 \times$ adjusted R-squared (WLS only), dimensionless \\
\hline pseudo $R^{2}$ & $100 \times$ pseudo R-squared (GLS only), dimensionless \\
\hline$S M E$ & Standard model error (GLS only), in percent \\
\hline$\sqrt{ }$ & Mark that qualifies (see text) the reliability of regression residuals \\
\hline
\end{tabular}

Figure 2. Summary of variables and regression diagnostics for regional equations. GLS, Generalized Least Squares; WLS, Weighted Least Squares.

\section{Weighted Least-Squares Regression}

The regionalized regression equations for computing the annual-and monthly-mean streamflow equations and the equations for the flow-duration curve were developed using WLS regression. For the regional regression analysis reported here, WLS regression was preferred over GLS regression because GLS regression was specifically developed for use with $T$-year type streamflow statistics and is difficult to extend to other streamflow statistics. The weights used for the WLS regression for the annual-mean and monthlymean equations are based on the variance of the logarithms of the observed values. Specifically, stations with lesser variance received greater weighting. The weights for flowduration equations are based on length of available record in years. The equations for estimating monthly- and annualmean streamflows and flow-duration curves for four of the five hydrologic regions are listed in figures 3-7. Equations were not developed for the Plains hydrologic region for reasons outlined in section "Selection of Stations for Other Streamflow Statistics."

The figures also provide appropriate WLS-regression diagnostics for each equation. These include the standard error of prediction and the adjusted R-squared statistic. Each equation is statistically significant with a p-value less than 0.01 . Readers are directed to standard texts on regression such as Helsel and Hirsch (2002) or Montgomery and others (2001) for details. Similar to the GLS-regression equations, the ranges of the predictor variables also are listed in the figures.

\section{Discussion of Selection of Variables}

The six selected basin and climatic characteristics were examined as predictor variables for the regression equations reported in figures 3-7. Regression-equation development is an inherently iterative process, and the modeler must 
balance several competing objectives, including the tradeoff between number of variables to include in the equation and the degrees of freedom (data points) used to estimate regression coefficients.

One goal in the early stages of regression-equation development is to identify and select those basin and climatic characteristics that have a statistically significant effect on the dependent variables and to generally exclude those predictor variables having limited influence. For example, $P$ and $E$ are strongly coupled in the study area in which the latter influences the former through orographic effects that enhance the formation of precipitation; hence, it is not always optimal to have both variables present in an equation. Other variables can be selected on professional judgment and logic and confirmed by statistical significance. For example, $A$ is a vital predictor variable for streamflow statistics and is the only common variable used in all regional equations developed by Kircher and others (1985) and Vaill (1999). For the regression equations reported here, $A$ is similarly included.

The selection of variables also is influenced by various regression diagnostics such as statistical significance (as measured by p-values), residual standard error, the distribution of regression residuals, several coefficient-of-determination statistics (R-squared statistics) or R-squared-like statistics (pseudo R-squared for the GLS regression; see Griffis and Stedinger [2007]) and others. Selection of variables can also be influenced by the preference to maintain a consistent structural form to a set of estimating equations in order to help ensure physically consistent estimates. By maintaining a regionally consistent set of included variables in the equations with ensembles of $T$-year peak streamflow, situations such as the 50-year peak streamflow being estimated as less than the 25 -year peak streamflow are virtually eliminated. By maintaining a regionally consistent set of included variables in the equation ensembles for monthly streamflow, a smooth monthto-month variation in estimated streamflow, which is presumably more reflective of actual seasonal variations, is provided.

\section{Application and Limitations of Regional Regression Equations}

Application and limitations of the regional regression equations in figures 3-7 exist. The number of equations reported here is too large for a comprehensive discussion of the subtle details of each. However, all of the equations reported in the figures are statistically significant, with a p-value less than 0.01 , and represent reasonably reliable equations that were developed from USGS data, available sample sizes, and techniques that are within the scope of the report.

The authors have assessed each equation and assigned a qualification as a "boxed check mark" to each equation based on visual interpretation of the residual plots (not reported here) for each equation. A boxed check mark is provided in figures 3-7 for each equation for which no bias was identified. A boxed check mark is not provided for those equations for which the residual plots indicate that bias (as either overestimation or underestimation) could exist for some combinations of basin and climatic characteristics. Such biases are indicated by nonlinear relations in the residual plots or the presence of heteroscedasticity (nonconstant variance). The magnitude and direction of bias are difficult to systematically quantify and mitigate for each of those equations within the scope of this report. For each of the equations listed in the figures, however, the qualification provides a mechanism by which the potential for biased estimation can be communicated to practitioners.

All streamflow statistics estimated from the equations are considered to be associated with natural basin and climatic characteristics and are not representative of anthropogenically influenced conditions, such as site-specific situations involving factors like the placement or removal of a dam or substantial diversion and return flows. In general, the equations and their associated errors are most valid for ungaged watersheds with basin and climatic characteristics well within the ranges of the predictor variables for each equation. Estimates and associated errors for ungaged sites having basin or climate characteristics outside the reported range of the independent variables are less applicable and could be erroneous. As a result, consideration of these and other limitations requires substantial judgment on the part of the practitioners for reliable application of the regional equations for estimation of natural streamflow statistics in Colorado.

The USGS has developed a Web-based computer program called Streamstats (Ries and others, 2004). The software facilitates the computation of streamflow statistics using regional regression equations or other procedures that have been published previously. Streamstats allows the user to obtain streamflow statistics for both gaged and ungaged sites by selecting a specific stream location on a map interface. If the location of interest lacks a station, the algorithms in Streamstats delineate the watershed for the location, compute basin and climatic characteristics, and provide estimates of the streamflow statistics using the available regression equations. In general, the computational algorithms used to generate basin and climatic characteristics for the regression equations reported here are consistent with those of Streamstats. As a result, predictor variables used to develop the equations presented in this report are compatible for use with Streamstats, which represents a unique contribution to ungaged-site, regional-streamflow estimation in Colorado. Finally, regression equations reported here for Colorado have been included in Streamstats to ease the computation of estimates of streamflow statistics for practitioners. 


\section{Peak Streamflow Equations for Mountain Hydrologic Region}

Generalized least-squares (GLS) regression, 141 stations

Approximate range of predictor variables

A: 1-1,060 square miles; $S: 7.6-60.2$ percent; and $P: 18-47$ inches

$$
\begin{array}{lllll}
Q_{2}=10^{-2.05} A^{0.78} S^{0.17} P^{2.10} & \sqrt{ } & S E P=49, & \operatorname{pseudo} R^{2}=83, & S M E=48, \\
Q_{5}=10^{-1.51} A^{0.77} S^{0.16} P^{1.85} & \sqrt{ } & S E P=44, & \operatorname{pseudo} R^{2}=86, & S M E=43, \\
Q_{10}=10^{-1.20} A^{0.77} S^{0.14} P^{1.71} & \sqrt{ } & S E P=41, & \operatorname{pseudo} R^{2}=87, & S M E=40, \\
Q_{25}=10^{-0.90} A^{0.75} S^{0.16} P^{1.55} & \sqrt{ } & S E P=40, & \operatorname{pseudo} R^{2}=88, & S M E=39, \\
Q_{50}=10^{-0.68} A^{0.75} S^{0.16} P^{1.45} & \sqrt{ } & S E P=39, & \operatorname{pseudo} R^{2}=88, & S M E=37, \\
Q_{100}=10^{-0.46} A^{0.75} S^{0.14} P^{1.35} & \sqrt{ } & S E P=36, & \operatorname{pseudo} R^{2}=90, & S M E=34, \\
Q_{200}=10^{-0.28} A^{0.75} S^{0.13} P^{1.26} & \sqrt{ } & S E P=36, & \operatorname{pseudo} R^{2}=90, & S M E=34, \text { and } \\
Q_{500}=10^{-0.06} A^{0.74} S^{0.15} P^{1.14} & \sqrt{ } & S E P=33, & \operatorname{pseudo} R^{2}=91, & S M E=31 .
\end{array}
$$

\section{Minimum Streamflow for Mountain Hydrologic Region}

Generalized least-squares (GLS) regression, 124, 118, and 111 stations

Approximate range of predictor variables

A: 1-1,060 square miles; $P$ : $18-47$ inches; and $E: 8,600-12,000$ feet elevation

$$
\begin{array}{lllll}
{ }_{7} Q_{2}^{\min }=10^{-26.56} A^{1.13} P^{1.26} E^{5.85} & \sqrt{ } & S E P=89, & \text { pseudo } R^{2}=80, & S M E=87, \\
{ }_{7} Q_{10}^{\min }=10^{-33.76} A^{1.20} P^{2.25} E^{7.20} & \sqrt{ } \quad S E P=153, & \text { pseudo } R^{2}=67, & S M E=148, \text { and } \\
{ }_{7} Q_{50}^{\min }=10^{-18.56} A^{1.17} P^{1.86} E^{3.56} & \sqrt{ } \quad S E P=126, & \text { pseudo } R^{2}=72, & S M E=122 .
\end{array}
$$

\section{Maximum Streamflow for Mountain Hydrologic Region}

Generalized least-squares (GLS) regression, 130 stations

Approximate range of predictor variables

$A: 1-1,060$ square miles and $P: 18-47$ inches

$$
\begin{aligned}
& { }_{7} Q_{2}^{\max }=10^{-1.99} A^{0.82} P^{2.08} \quad \sqrt{ } \quad S E P=46, \quad \operatorname{pseudo} R^{2}=86, \quad S M E=45, \\
& { }_{7} Q_{10}^{\max }=10^{-1.38} A^{0.85} P^{1.78} \quad \sqrt{ } \quad S E P=35, \quad \text { pseudo } R^{2}=92, \quad S M E=34 \text {, and } \\
& { }_{7} Q_{50}^{\max }=10^{-0.94} A^{0.85} P^{1.57} \quad \sqrt{ } \quad S E P=31, \quad \text { pseudo } R^{2}=94, \quad S M E=30 .
\end{aligned}
$$

Summary of Variables, Units, and Regression Diagnostics is shown in figure 2.

Figure 3. Regional regression equations for the Mountain hydrologic region. 
Annual and Monthly Mean Streamflow for Mountain Hydrologic Region

Weighted least-squares (WLS) regression, 129 stations

Approximate range of predictor variables

A: $1-1,060$ square miles and $P: 18-47$ inches

\begin{tabular}{|c|c|c|c|}
\hline$Q_{\mathrm{ann}}=10^{-2.31} A^{0.88} P^{1.71}$ & $\sqrt{ }$ & $S E P=33$, & $\operatorname{adj} R^{2}=93$, \\
\hline$Q_{\text {oct }}=10^{-3.29} A^{0.97} P^{1.98}$ & $\sqrt{ }$ & $S E P=45$ & $\operatorname{adj} R^{2}=89$ \\
\hline$Q_{\mathrm{nov}}=10^{-3.14} A^{0.98} P^{1.78}$ & $\sqrt{ }$ & $S E P=46$ & $\operatorname{adj} R^{2}=90$ \\
\hline$Q_{\mathrm{dec}}=10^{-2.90} A^{1.00} P^{1.52}$ & $\sqrt{1}$ & $S E P=47$, & $\operatorname{adj} R^{2}=89$ \\
\hline$Q_{\text {jan }}=10^{-2.99} A^{1.02} P^{1.53}$ & $\sqrt{ }$ & $S E P=50$, & $\operatorname{adj} R^{2}=88$ \\
\hline$Q_{\mathrm{feb}}=10^{-2.92} A^{1.02} P^{1.46}$ & $\sqrt{ }$ & $S E P=51$, & $\operatorname{adj} R^{2}=88$, \\
\hline$Q_{\mathrm{mar}}=10^{-2.87} A^{1.06} P^{1.42}$ & $\sqrt{ }$ & $S E P=49$ & $\operatorname{adj} R^{2}=89$ \\
\hline$Q_{\mathrm{apr}}=10^{-2.95} A^{1.14} P^{1.64}$ & $\sqrt{ }$ & $S E P=44$ & $\operatorname{adj} R^{2}=92$ \\
\hline$Q_{\text {may }}=10^{-1.60} A^{0.94} P^{1.44}$ & $\sqrt{ }$ & $S E P=46$ & $\operatorname{adj} R^{2}=87$ \\
\hline$Q_{\text {jun }}=10^{-1.29} A^{0.82} P^{1.52}$ & & $S E P=46$ & $\operatorname{adj} R^{2}=87$ \\
\hline$Q_{\text {jul }}=10^{-2.80} A^{0.84} P^{2.23}$ & & $S E P=76$ & $\operatorname{adj} R^{2}=72$ \\
\hline$Q_{\text {aug }}=10^{-2.73} A^{0.85} P^{1.91}$ & & $S E P=80$, & $\operatorname{adj} R^{2}=75$, and \\
\hline$Q_{\text {sep }}=10^{-2.93} A^{0.92} P^{1.84}$ & & $S E P=59$, & $\operatorname{adj} R^{2}=86$ \\
\hline
\end{tabular}

Flow-Duration Curve for Mountain Hydrologic Region

Weighted least-squares (WLS) regression, 129 stations

Approximate range of predictor variables

A: $1-1,060$ square miles and $P$ : $18-47$ inches

$\begin{array}{llll}Q_{10 \text { th }}=10^{-2.64} A^{0.89} P^{2.22} & \sqrt{ } & S E P=45, & \operatorname{adj} R^{2}=90, \\ Q_{25 \text { th }}=10^{-2.86} A^{0.96} P^{1.92} & \sqrt{ } & S E P=55, & \operatorname{adj} R^{2}=88, \\ Q_{50 \text { th }}=10^{-2.69} A^{0.98} P^{1.49} & \sqrt{ } & S E P=55, & \operatorname{adj} R^{2}=88, \\ Q_{75 \text { th }}=10^{-2.85} A^{1.01} P^{1.40} & \sqrt{ } & S E P=64, & \operatorname{adj} R^{2}=86, \text { and } \\ Q_{90 \text { th }}=10^{-3.46} A^{1.10} P^{1.59} & \sqrt{ } & S E P=85, & \operatorname{adj} R^{2}=82 .\end{array}$

Summary of Variables, Units, and Regression Diagnostics is shown in figure 2.

Figure 3. Regional regression equations for the Mountain hydrologic region.-Continued 
Peak Streamflow Equations for Northwest Hydrologic Region

Generalized least-squares (GLS) regression, 90 stations

Approximate range of predictor variables

$A$ : 1-5,250 square miles; $A_{7500}: 0-100$ percent, and $P: 8-49$ inches

$$
\begin{aligned}
& Q_{2}=10^{-1.15} A^{0.75} A_{7500}^{-0.41} P^{2.15} \\
& Q_{5}=10^{-0.49} A^{0.76} A_{7500}^{-0.54} P^{2.00} \\
& Q_{10}=10^{-0.10} A^{0.75} A_{7500}^{-0.63} P^{1.93} \\
& Q_{25}=10^{0.35} A^{0.75} A_{7500}^{-0.72} P^{1.82} \\
& Q_{50}=10^{0.65} A^{0.74} A_{7500}^{-0.77} P^{1.74} \\
& Q_{100}=10^{0.93} A^{0.74} A_{7500}^{-0.81} P^{1.65} \\
& Q_{200}=10^{1.20} A^{0.73} A_{7500}^{-0.85} P^{1.56} \\
& Q_{500}=10^{1.53} A^{0.72} A_{7500}^{-0.88} P^{1.44}
\end{aligned}
$$

\section{Minimum Streamflow for Northwest Hydrologic Region}

Generalized least-squares (GLS) regression, 52, 47, and 42 stations

Approximate range of predictor variables

A: 5-5,250 square miles; $E$ : 6,880-10,480 feet
${ }_{7} Q_{2}^{\min }=10^{-34.73} A^{0.83} E^{8.56}$
$S E P=212, \quad \operatorname{pseudo} R^{2}=42$,
$S M E=199$,
${ }_{7} Q_{10}^{\min }=10^{-38.52} A^{0.90} E^{9.42}$
$S E P=280, \quad \operatorname{pseudo} R^{2}=41$,
$S M E=259$, and
${ }_{7} Q_{50}^{\min }=10^{-36.44} A^{0.94} E^{8.84}$
$S E P=338$,
pseudo $R^{2}=39$,
$S M E=307$.

\section{Maximum Streamflow for Northwest Hydrologic Region}

Generalized least-squares (GLS) regression, 59 stations

Approximate range of predictor variables

A: 5-5,250 square miles, $P: 8-49$ inches, and $A_{7500}: 0-100$ percent
${ }_{7} Q_{2}^{\max }=10^{-7.19} A^{1.03} P^{3.60} A_{7500}^{1.25}$
$S E P=86, \quad$ pseudo $R^{2}=84$,
$S M E=82$,
${ }_{7} Q_{10}^{\max }=10^{-5.35} A^{1.00} P^{2.48} A_{7500}^{1.31}$
$S E P=59, \quad \operatorname{pseudo} R^{2}=89$,
$S M E=56$, and
${ }_{7} Q_{50}^{\max }=10^{-3.90} A^{0.94} P^{1.80} A_{7500}^{1.23}$
$S E P=51$
pseudo $R^{2}=90$,
$S M E=48$.

Summary of Variables, Units, and Regression Diagnostics is shown in figure 2.

Figure 4. Regional regression equations for the Northwest hydrologic region. 
Annual and Monthly Mean Streamflow for Northwest Hydrologic Regio।

Weighted least-squares (WLS) regression, 56 stations

Approximate range of predictor variables

$A: 1-5,250$ square miles and $P: 8-49$ inches

$\begin{array}{llll}Q_{\text {ann }}=10^{-4.98} A^{0.95} P^{3.32} & \sqrt{ } & S E P=55, & \operatorname{adj} R^{2}=88, \\ Q_{\text {oct }}=10^{-4.32} A^{1.01} P^{2.54} & & S E P=94, & \operatorname{adj} R^{2}=78, \\ Q_{\text {nov }}=10^{-4.68} A^{1.08} P^{2.62} & & S E P=83, & \operatorname{adj} R^{2}=83, \\ Q_{\text {dec }}=10^{-4.53} A^{1.07} P^{2.46} & & S E P=79, & \operatorname{adj} R^{2}=82, \\ Q_{\text {jan }}=10^{-4.73} A^{1.05} P^{2.62} & & S E P=85, & \operatorname{adj} R^{2}=81, \\ Q_{\text {feb }}=10^{-4.63} A^{1.14} P^{2.46} & & S E P=77, & \operatorname{adj} R^{2}=85, \\ Q_{\text {mar }}=10^{-4.39} A^{1.14} P^{2.36} & & S E P=68, & \operatorname{adj} R^{2}=90, \\ Q_{\text {apr }}=10^{-4.77} A^{1.06} P^{3.02} & \sqrt{ } & S E P=84, & \operatorname{adj} R^{2}=85, \\ Q_{\text {may }}=10^{-4.54} A^{0.96} P^{3.40} & \sqrt{ } & S E P=71, & \operatorname{adj} R^{2}=84, \\ Q_{\text {jun }}=10^{-6.90} A^{1.00} P^{4.87} & \sqrt{ } & S E P=80, & \operatorname{adj} R^{2}=82, \\ Q_{\text {jul }}=10^{-4.37} A^{0.83} P^{3.09} & & S E P=75, & \operatorname{adj} R^{2}=68, \\ Q_{\text {aug }}=10^{-3.03} A^{0.84} P^{1.98} & & S E P=90, & \operatorname{adj} R^{2}=67, \operatorname{and} \\ Q_{\text {sep }}=10^{-2.62} A^{0.92} P^{1.55} & & S E P=104, & \operatorname{adj} R^{2}=70 .\end{array}$

Flow-Duration Curve for Northwest Hydrologic Region

Weighted least-squares (WLS) regression, 62 stations

Approximate range of predictor variables

A: $1-5,250$ square miles and $P: 8-49$ inches

$\begin{array}{llll}Q_{10 \text { th }}=10^{-6.03} A^{1.03} P^{4.23} & \downarrow & S E P=73, & \operatorname{adj} R^{2}=86, \\ Q_{25 \text { th }}=10^{-5.86} A^{1.05} P^{3.72} & \checkmark & S E P=77, & \operatorname{adj} R^{2}=85, \\ Q_{50 \text { th }}=10^{-6.07} A^{1.05} P^{3.61} & \checkmark & S E P=83, & \operatorname{adj} R^{2}=83, \\ Q_{75 \text { th }}=10^{-6.91} A^{1.07} P^{3.98} & \checkmark & S E P=100, & \operatorname{adj} R^{2}=80, \text { and } \\ Q_{90 \text { th }}=10^{-8.32} A^{1.06} P^{4.80} & \checkmark & S E P=154, & \operatorname{adj} R^{2}=70 .\end{array}$

Summary of Variables, Units, and Regression Diagnostics is shown in figure 2.

Figure 4. Regional regression equations for the Northwest hydrologic section.-Continued 
Peak Streamflow Equations for Rio Grande Hydrologic Region

Generalized least-squares (GLS) regression, 44 stations

Approximate range of predictor variables

A: 2-517 square miles and $P: 19-45$ inches

\begin{tabular}{|c|c|c|c|c|}
\hline$Q_{2}=10^{-3.00} A^{1.00} P^{2.46}$ & $\sqrt{ }$ & $S E P=67$ & pseudo $R^{2}=80$ & $S M E=64$ \\
\hline$Q_{5}=10^{-2.04} A^{0.95} P^{2.02}$ & V & $S E P=57$ & pseudo $R^{2}=83$ & $S M E=55$ \\
\hline$Q_{10}=10^{-1.55} A^{0.93} P^{1.80}$ & $\sqrt{V}$ & $S E P=54$ & pseudo $R^{2}=84$ & $S M E=51$ \\
\hline$Q_{25}=10^{-1.01} A^{0.91} P^{1.55}$ & $\sqrt{ }$ & $S E P=52$ & pseudo $R^{2}=84$ & $S M E=49$ \\
\hline$Q_{50}=10^{-0.66} A^{0.89} P^{1.39}$ & $\sqrt{ }$ & $S E P=51$ & pseudo $R^{2}=85$ & $S M E=48$ \\
\hline$Q_{100}=10^{-0.19} A^{0.87} P^{1.17}$ & $\sqrt{ }$ & $S E P=51$ & pseudo $R^{2}=85$ & $S M E=48$ \\
\hline$Q_{200}=10^{-0.03} A^{0.86} P^{1.11}$ & $\sqrt{ }$ & $S E P=52$ & pseudo $R^{2}=84$ & $S M E=49$, and \\
\hline$Q_{500}=10^{0.52} A^{0.84} P^{0.85}$ & $\sqrt{ }$ & $S E P=54$ & pseudo $R^{2}=84$ & $S M E=49$ \\
\hline
\end{tabular}

\section{Minimum Streamflow for Rio Grande Hydrologic Region}

Generalized least-squares (GLS) regression, 28, 27, and 24 stations

Approximate range of predictor variables

$A$ : 13-517 square miles and $E: 8,600-12,000$ feet elevation

$$
\begin{aligned}
& { }_{7} Q_{2}^{\min }=10^{-44.17} A^{1.03} E^{10.71} \quad \downarrow \quad S E P=111, \quad \text { pseudo } R^{2}=51, \quad S M E=103, \\
& { }_{7} Q_{10}^{\min }=10^{-46.35} A^{1.09} E^{11.15} \quad \sqrt{ } \quad S E P=189, \quad \text { pseudo } R^{2}=37, \quad S M E=170 \text {, and } \\
& { }_{7} Q_{50}^{\min }=10^{-49.44} A^{1.13} E^{11.88} \quad \sqrt{ } \quad S E P=165, \quad \text { pseudo } R^{2}=47, \quad S M E=148 .
\end{aligned}
$$

\section{Maximum Streamflow for Rio Grande Hydrologic Region}

Generalized least-squares (GLS) regression, 31 stations

Approximate range of predictor variables

A: $13-517$ square miles and $P: 19-45$ inches
${ }_{7} Q_{2}^{\max }=10^{-3.83} A^{0.98} P^{2.89}$

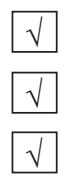
$S E P=81$,
pseudo $R^{2}=68$
$S M E=76$,
${ }_{7} Q_{10}^{\max }=10^{-2.22} A^{0.92} P^{2.11}$
$S E P=55$,
pseudo $R^{2}=77$,
$S M E=52$, and
${ }_{7} Q_{50}^{\max }=10^{-1.38} A^{0.86} P^{1.74}$
$S E P=31$
pseudo $R^{2}=94$,
$S M E=30$.

Summary of Variables, Units, and Regression Diagnostics is shown in figure 2.

Figure 5. Regional regression equations for the Rio Grande hydrologic region. 
Annual and Monthly Mean Streamflow for Rio Grande Hydrologic Region

Weighted least-squares (WLS) regression, 32 stations

Approximate range of predictor variables

A: 2-517 square miles and $E: 7,790-11,500$ feet

\begin{tabular}{|c|c|c|c|}
\hline$Q_{\mathrm{ann}}=10^{-33.58} A^{1.12} E^{8.24}$ & $\sqrt{ }$ & $S E P=73$ & $\operatorname{adj} R^{2}=72$ \\
\hline$Q_{\text {oct }}=10^{-32.88} A^{0.95} E^{8.04}$ & $\sqrt{ }$ & $S E P=55$ & $\operatorname{adj} R^{2}=72$ \\
\hline$Q_{\mathrm{nov}}=10^{-24.79} A^{0.90} E^{6.02}$ & $\sqrt{ }$ & $S E P=53$ & $\operatorname{adj} R^{2}=73$ \\
\hline$Q_{\mathrm{dec}}=10^{-17.71} A^{0.86} E^{4.26}$ & $\sqrt{ }$ & $S E P=63$ & $\operatorname{adj} R^{2}=68$ \\
\hline$Q_{\text {jan }}=10^{-13.34} A^{0.83} E^{3.18}$ & \begin{tabular}{|l|}
$\sqrt{ }$ \\
\end{tabular} & $S E P=64$ & $\operatorname{adj} R^{2}=66$ \\
\hline$Q_{\mathrm{feb}}=10^{-11.68} A^{0.87} E^{2.75}$ & \begin{tabular}{|l|}
$\sqrt{ }$ \\
\end{tabular} & $S E P=56$ & $\operatorname{adj} R^{2}=74$ \\
\hline$Q_{\text {mar }}=10^{-9.32} A^{0.97} E^{2.14}$ & $\sqrt{ }$ & $S E P=49$ & $\operatorname{adj} R^{2}=82$ \\
\hline$Q_{\text {apr }}=10^{-5.09} A^{1.10} E^{1.16}$ & \begin{tabular}{|l|}
$\sqrt{ }$ \\
\end{tabular} & $S E P=79$ & $\operatorname{adj} R^{2}=75$ \\
\hline$Q_{\text {may }}=10^{-37.25} A^{1.26} E^{9.23}$ & $\sqrt{ }$ & $S E P=84$ & $\operatorname{adj} R^{2}=71$ \\
\hline$Q_{\text {jun }}=10^{-61.30} A^{1.23} E^{15.22}$ & \begin{tabular}{|l|}
$\sqrt{ }$ \\
\end{tabular} & $S E P=84$ & $\operatorname{adj} R^{2}=74$ \\
\hline$Q_{\text {jul }}=10^{-53.30} A^{1.07} E^{13.17}$ & $\checkmark$ & $S E P=74$ & $\operatorname{adj} R^{2}=72$ \\
\hline$Q_{\text {aug }}=10^{-43.00} A_{1.03} E^{10.59}$ & $\sqrt{ }$ & $S E P=53$ & $\operatorname{adj} R^{2}=80$, and \\
\hline$Q_{\text {sep }}=10^{-41.49} A^{0.99} E^{10.19}$ & $\sqrt{ }$ & $S E P=57$ & $\operatorname{adj} R^{2}=75$ \\
\hline
\end{tabular}

\section{Flow-Duration Curve for Rio Grande Hydrologic Region}

Weighted least-squares (WLS) regression, 32 stations

Approximate range of predictor variables

A: 2-517 square miles and $E: 7,790-11,500$ feet
$Q_{10 \mathrm{th}}=10^{-32.35} A^{1.13} E^{8.04}$
$S E P=81$,
$\operatorname{adj} R^{2}=66$,
$Q_{25 \mathrm{th}}=10^{-41.33} A^{1.07} E^{10.18}$
$S E P=67$,
$\operatorname{adj} R^{2}=72$,
$Q_{50 \mathrm{th}}=10^{-38.61} A^{0.96} E^{9.46}$
$S E P=75$,
$\operatorname{adj} R^{2}=64$,
$Q_{75 \text { th }}=10^{-42.09} A^{0.90} E^{10.30}$
$S E P=112$,
$\operatorname{adj} R^{2}=48$, and
$Q_{90 \mathrm{th}}=10^{-50.71} A^{0.89} E^{12.42}$
$S E P=145$,
$\operatorname{adj} R^{2}=44$.

Summary of Variables, Units, and Regression Diagnostics is shown in figure 2.

Figure 5. Regional regression equations for the Rio Grande hydrologic region.-Continued 
Peak Streamflow Equations for Southwest Region

Generalized least-squares (GLS) regression, 78 stations

Approximate range of predictor variables

A: 1-4,390 square miles and $A_{7500: 0-100 \text { percent }}$

\begin{tabular}{|c|c|c|c|c|}
\hline$Q_{2}=10^{1.67} A^{0.64} A_{7500}^{-0.10}$ & \begin{tabular}{|l|}
$\sqrt{ }$ \\
\end{tabular} & $S E P=90$ & pseudo $R^{2}=70$ & $S M E=87$ \\
\hline$Q_{5}=10^{2.13} A^{0.62} A_{7500}^{-0.19}$ & $\sqrt{ }$ & $S E P=71$ & pseudo $R^{2}=75$ & $S M E=69$, \\
\hline$Q_{10}=10^{2.36} A^{0.61} A_{7500}^{-0.23}$ & $\sqrt{ }$ & $S E P=67$ & pseudo $R^{2}=77$ & $S M E=64$ \\
\hline$Q_{25}=10^{2.61} A^{0.60} A_{7500}^{-0.27}$ & $\sqrt{1}$ & $S E P=66$ & pseudo $R^{2}=78$ & $S M E=63$ \\
\hline$Q_{50}=10^{2.77} A^{0.59} A_{7500}^{-0.30}$ & $\sqrt{ }$ & $S E P=67$ & pseudo $R^{2}=78$ & $S M E=63$ \\
\hline$Q_{100}=10^{2.91} A^{0.59} A_{7500}^{-0.33}$ & $\sqrt{ }$ & $S E P=69$ & pseudo $R^{2}=78$ & $S M E=65$, \\
\hline$Q_{200}=10^{3.04} A^{0.58} A_{7500}^{-0.36}$ & $\sqrt{ }$ & $S E P=71$ & pseudo $R^{2}=77$ & $S M E=67$, and \\
\hline$Q_{500}=10^{3.21} A^{0.58} A_{7500}^{-0.39}$ & $\sqrt{ }$ & $S E P=75$ & pseudo $R^{2}=77$ & $S M E=70$. \\
\hline
\end{tabular}

Minimum Streamflow for Southwest Region

Generalized least-squares (GLS) regression, 46, 37, and 33 stations

Approximate range of predictor variables

A: 4-4,390 square miles, $P$ : 10-51 inches, $E$ : 5,600-11,600 feet

$\begin{array}{lllll}{ }_{7} Q_{2}^{\text {min }}=10^{-22.24} A^{1.16} P^{1.51} E^{4.65} & \sqrt{ } & S E P=226, & \text { pseudo } R^{2}=67, & S M E=207, \\ { }_{7} Q_{10}^{\text {min }}=10^{-18.74} A^{0.97} P^{1.35} E^{3.88} & \sqrt{ } & S E P=255, & \text { pseudo } R^{2}=52, & S M E=226, \text { and } \\ { }_{7} Q_{50}^{\text {min }}=10^{-26.29} A^{0.49} P^{0.11} E^{6.45} & \sqrt{ } & S E P=354, & \text { pseudo } R^{2}=33, & S M E=300 .\end{array}$

\section{Maximum Streamflow for Southwest Region}

Generalized least-squares (GLS) regression, 59 stations

Approximate range of predictor variables

A: 4-4,390 square miles and $P: 10-51$ inches
${ }_{7} Q_{2}^{\max }=10^{-4.07} A^{0.99} P^{3.10}$
$\checkmark \quad S E P=64$,
pseudo $R^{2}=88$,
$S M E=61$,
${ }_{7} Q_{10}^{\max }=10^{-2.68} A^{0.93} P^{2.44}$
$S E P=43$,
pseudo $R^{2}=93$,
$S M E=40$, and
${ }_{7} Q_{50}^{\max }=10^{-1.86} A^{0.89} P^{2.03}$
$S E P=33$,
pseudo $R^{2}=95$,
$S M E=30$.

Summary of Variables, Units, and Regression Diagnostics is shown in figure 2.

Figure 6. Regional regression equations for the Southwest hydrologic region. 
Annual and Monthly Mean Streamflow for Southwest Region

Weighted least-squares (WLS) regression, 57 stations

Approximate range of predictor variables

A: $1-4,390$ square miles and $P: 10-51$ inches

\begin{tabular}{|c|c|c|c|}
\hline$Q_{\mathrm{ann}}=10^{-4.37} A^{0.91} P^{2.98}$ & & $S E P=60$ & $\operatorname{adj} R^{2}=85$ \\
\hline$Q_{\mathrm{oct}}=10^{-4.80} A^{1.00} P^{2.89}$ & $\sqrt{ }$ & $S E P=106$ & $\operatorname{adj} R^{2}=77$ \\
\hline$Q_{\mathrm{nov}}=10^{-4.59} A^{0.91} P^{2.78}$ & $\checkmark$ & $S E P=80$ & $\operatorname{adj} R^{2}=76$ \\
\hline$Q_{\mathrm{dec}}=10^{-4.22} A^{0.91} P^{2.45}$ & $\sqrt{ }$ & $S E P=75$ & $\operatorname{adj} R^{2}=75$ \\
\hline$Q_{\mathrm{jan}}=10^{-4.28} A^{0.96} P^{2.39}$ & \begin{tabular}{|l|}
$V$ \\
\end{tabular} & $S E P=77$ & $\operatorname{adj} R^{2}=77$ \\
\hline$Q_{\mathrm{feb}}=10^{-3.97} A^{0.98} P^{2.18}$ & $\checkmark$ & $S E P=58$ & $\operatorname{adj} R^{2}=83$ \\
\hline$Q_{\mathrm{mar}}=10^{-3.79} A^{1.00} P^{2.12}$ & $\checkmark$ & $S E P=47$ & $\operatorname{adj} R^{2}=82$ \\
\hline$Q_{\text {apr }}=10^{-4.98} A^{1.12} P^{3.11}$ & & $S E P=50$ & $\operatorname{adj} R^{2}=95$ \\
\hline$Q_{\text {may }}=10^{-5.16} A^{1.01} P^{3.63}$ & $\sqrt{ }$ & $S E P=62$ & $\operatorname{adj} R^{2}=88$ \\
\hline$Q_{\text {jun }}=10^{-6.13} A^{1.05} P^{4.30}$ & & $S E P=121$ & $\operatorname{adj} R^{2}=85$ \\
\hline$Q_{\text {jul }}=10^{-5.19} A^{0.91} P^{3.58}$ & & $S E P=180$ & $\operatorname{adj} R^{2}=63$ \\
\hline$Q_{\text {aug }}=10^{-4.60} A^{0.94} P^{2.95}$ & & $S E P=119$ & $\operatorname{adj} R^{2}=64$, ar \\
\hline$Q_{\text {sen }}=10^{-8.72} A^{0.98} P^{5.46}$ & & $S E P=120$ & $\operatorname{adj} R^{2}=79$ \\
\hline
\end{tabular}

Flow-Duration Curve for Southwest Region

Weighted least-squares (WLS) regression, 57 stations

Approximate range of predictor variables

A: $1-4,390$ square miles and $P: 10-51$ inches

\begin{tabular}{|c|c|c|c|}
\hline$Q_{10 \text { th }}=10^{-5.44} A^{1.02} P^{3.79}$ & $\sqrt{ }$ & $S E P=79$ & $\operatorname{adj} R^{2}=85$ \\
\hline$Q_{25 \text { th }}=10^{-5.27} A^{1.00} P^{3.40}$ & $\checkmark$ & $S E P=96$ & $\operatorname{adj} R^{2}=78$ \\
\hline$Q_{50 \text { th }}=10^{-5.08} A^{0.98} P^{3.01}$ & $\checkmark$ & $S E P=98$ & $\operatorname{adj} R^{2}=75$ \\
\hline$Q_{7 \text { th }}=10^{-5.99} A^{1.02} P^{3.37}$ & $\checkmark$ & $S E P=100$ & $\operatorname{adj} R^{2}=78$, and \\
\hline$Q_{90 \text { th }}=10^{-7.30} A^{1.01} P^{4.11}$ & $\checkmark$ & $S E P=148$ & $\operatorname{adj} R^{2}=72$ \\
\hline
\end{tabular}

Summary of Variables, Units, and Regression Diagnostics is shown in figure 2.

Figure 6. Regional regression equations for the Southwest hydrologic region.-Continued 
Peak Streamflow Equations for Plains Hydrologic Region

Generalized least-squares (GLS) regression, 69 stations

Approximate range of predictor variables

$A$ : $0.5-2,930$ square miles and ${ }_{6} P_{100}: 2.4-5.1$ inches

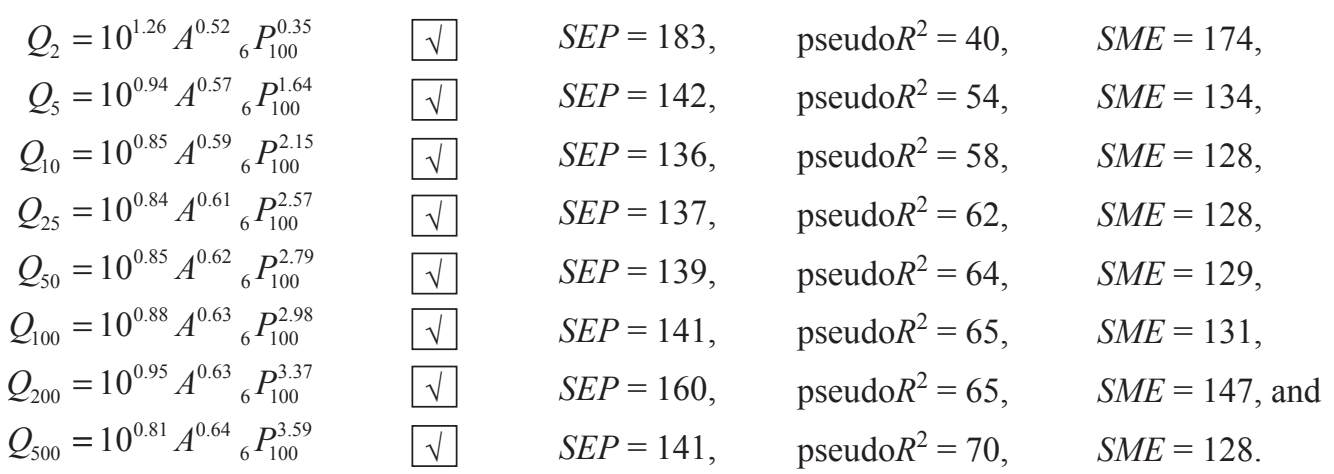

Summary of Variables, Units, and Regression Diagnostics is shown in figure 2.

Figure 7. Regional regression equations for the Plains hydrologic region.

\section{Summary}

The U.S. Geological Survey (USGS), in cooperation with the Colorado Water Conservation Board and the Colorado Department of Transportation, developed regional regression equations for estimation of various streamflow statistics that are representative of natural streamflow conditions in Colorado. Accurate estimates of various streamflow statistics are important for water-resource management, stream-related structural design, stream-hazard identification, and water-quality management. Streamflow statistics routinely are needed for sites in ungaged watersheds (ungaged sites) that lack nearby streamflow-gaging stations (stations) from which streamflow statistics could be directly computed. Regional regression equations are a common tool used to estimate streamflow statistics at ungaged sites across the Nation and Colorado.

Regional regression equations are based on statistical relations between (1) streamflow statistics of interest computed from applicable records of the stations and (2) basin and climatic characteristics, for which data are typically readily available. The use of regional equations generally represents a reliable and costeffective means for estimating streamflow statistics at ungaged sites, along with expressions of predictive uncertainty.

The purpose of this report is to present regional regression equations by hydrologic region for estimation of streamflow statistics for naturally flowing streams in Colorado. The equations were developed using the statistical relations between streamflow statistics (response variables) and basin and climatic characteristics (predictor variables).

For each of five hydrologic regions in Colorado, peakstreamflow equations that are based on peak-streamflow data from selected stations are presented for the 2-, 5-, 10-, 25-, 50-,
100-, 200- and 500-year instantaneous-peak streamflows (peak streamflow). For four of the five hydrologic regions, minimum and maximum 7-day streamflow (7-day, $T$-year) equations based on daily-mean streamflow data from selected stations are presented for 7-day minimum 2-, 10-, and 50-year streamflows, as well as for 7-day maximum 2-, 10-, and 50-year streamflows. Other equations presented for the same four hydrologic regions include those for estimation of annual- and monthly-mean streamflow and streamflow-duration statistics for exceedances of $10,25,50,75$, and 90 percent. The streamflow-duration statistics collectively are referred to as the "flow-duration curve." The flow-duration values, presented in this report as Q10th, Q25th, Q50th, Q75th, and Q90th, indicate the percentage of days that a given daily-mean streamflow is exceeded. For example, a 10-percent flow-duration value (Q10th) is calculated as the daily-mean streamflow at a given station that is exceeded 10 percent of the time or 10 percent of the days. Finally, this report also describes some limitations associated with the equations, including an assessment of prediction errors and assessment of equation applicability for ungaged sites.

The regional equations for peak-streamflow estimation were developed using annual peak-streamflow data collected through water year 2006 (October 1, 2005, through September 30, 2006). The remaining equations, with the exception of the 7-day, $T$-year minimum streamflow equations, were developed using data through water year 2007. The 7-day, $T$-year minimum streamflow equations were developed using data collected through climate year 2007 (April 1, 2006, through March 31, 2007).

The study was limited to the use of several selected basin and climatic characteristics including drainage area; mean watershed elevation; mean watershed slope; percentage of drainage area above 7,500 feet of elevation; mean annual precipitation; and 6-hour, 100-year precipitation. 
The computation of the regional regression equations used multilinear regression to define statistical relations between each of the streamflow statistics and two or more basin and climatic characteristics. For the investigation, logarithmic transformations (base-10) were made on all streamflow statistics and basin and climatic characteristics prior to the development of the regression equations. The data were logarithmically transformed to increase linearity between the regression variables and to acquire a constant variance of the regression residuals.

For the investigation, two forms of related regression techniques were used-generalized least-squares (GLS) and weighted least-squares (WLS) regression analyses. GLS regression was used exclusively to compute equations for peak-streamflow and 7-day minimum and maximum streamflow estimation. WLS was used exclusively to compute equations for annual- and monthlymean streamflow and flow-duration curve estimation.

The regionalized regression equations for computing the peak-, minimum-, and maximum-streamflow equations were developed using the GLS method. For the regional streamflowfrequency regression analysis reported here, GLS regression was preferred over WLS regression because GLS is an extension of WLS that accounts for cross correlation between response variables. WLS regression only accounts for differences in station record length and uncertainty in the station-specific streamflow statistics and assumes independence between response variables.

WLS regression was used for the development of regionalized regression equations for computing the annual- and monthly-mean streamflow equations and the equations for the flow-duration curve. WLS regression was preferred over GLS regression because GLS regression was specifically developed for use with $T$-year type streamflow statistics and is difficult to extend to other streamflow statistics. The weights used for the WLS regression for the annual-mean and monthly-mean equations are based on the variance of the logarithms of the observed values. The weights for flow-duration equations are based on length of available record in years.

Appropriate diagnostics for each of the equations are reported. These are standard error of prediction in percent (GLS and WLS), the adjusted R-squared statistic in percent (WLS only), the pseudo R-squared statistics in percent (GLS only), and the standard model error in percent (GLS only). Each of the equations is statistically significant with p-values less than 0.01 . The ranges of the predictor variables also are provided. These ranges can be used to assess equation applicability. The applicability of the equations for ungaged sites with basin and climate characteristics outside the range of the predictor variables is questionable.

Application and limitations of the regional regression equations exist. All of the equations reported in the figures are statistically significant and represent reasonably reliable equations that have been developed from USGS data, available sample sizes, and techniques that are within the scope of the report.

The authors have assessed each equation and assigned a qualification to each equation based on visual interpretation of the residual plots (not reported here) for each equation. For some equations the residual plots indicate that bias (as either overestimation or underestimation) could exist for some combinations of basin and climatic characteristics. Such biases are indicated by nonlinear relations in the residual plots or the presence of heteroscedasticity (nonconstant variance). The magnitude and direction of bias are difficult to systematically quantify and mitigate for each of those equations within the scope of this report. For each of the equations listed in the figures, however, the qualification provides a mechanism by which the potential for biased estimation can be communicated to practitioners.

All streamflow statistics estimated from the equations are considered to be associated with natural basin and climatic characteristics and are not representative of anthropogenically influenced conditions, such as site-specific situations involving factors like the placement or removal of a dam or substantial diversion and return flows. In general, the equations and their associated errors are most valid for ungaged watersheds with basin and climatic characteristics well within the ranges of the predictor variables for each equation. Estimates and associated errors for ungaged sites having basin or climate characteristics outside the reported range of the independent variables are less applicable and could be erroneous. As a result, consideration of these and other limitations requires substantial judgment on the part of the practitioner for reliable application of the regional equations for estimation of natural streamflow statistics in Colorado.

\section{Acknowledgments}

The authors recognize John England of the Bureau of Reclamation and William H. Asquith, Kenny Eng, Robert Jarrett, Terry Kenney, Julie Kiang, and Karen Ryberg of the USGS for providing invaluable technical guidance and review for this study and report.

\section{Selected References}

Berenbrock, Charles, 2002, Estimating the magnitude of peak flows at selected recurrence intervals for streams in Idaho: U.S. Geological Survey Water-Resources Investigations Report 2002-4170, 59 p.

Bonnin, G., Martin, T., Lin, D., Parzybok, B., Yekta, M., and Riley, D., 2006, Precipitation frequency Atlas of the United States: National Weather Service, National Oceanic and Atmospheric Administration Atlas 14, Volume III, accessed August 16, 2007, at http://www.nws.noaa.gov/oh/hdsc/ PF_documents/Atlas2_Volume3.pdf

Colorado Geological Survey, 2003, Ground water atlas of Colorado: Colorado Geological Survey Special Publication Number 53, 210 p.

Colorado State University, 2008, Precipitation map of Colorado: Fort Collins, Colorado State University, accessed July 28, 2008 at http://waterknowledge.colostate.edu/prcp_map.htm 
Daly, Christopher, Nielson, R.P., and Phillips, D.L., 1994, A statistical-topographic model for mapping climatological precipitation over mountainous terrain: Journal of Applied Meteorology, v. 33, no. 2, p. 140-158, accessed August 15, 2007 at http://www.prism.oregonstate.edu/products/

Daymet Project, daily surface weather and climatological summaries at $1 \mathrm{~km}$ resolution over the conterminous United States, accessed August 15, 2007, at http:://www.daymet.org

Fenneman, N.M., 1931, Physiography of the western United States: McGraw-Hill, Inc., New York, 534 p.

Flynn, K.M., Kirby, W.H., and Hummel, P.R., 2006, User's manual for program PeakFQ, Annual flood frequency analysis using Bulletin 17B guidelines: U.S. Geological Survey Techniques and Methods book 4, chap. B4, 42 p.

Griffis, V.W., and Stedinger, J.R., 2007, The use of GLS regression in regional hydrologic analyses: Journal of Hydrology, v. 344, p. 82-95.

Headman, E.R., Moore, D.O., and Livingston, R.K., 1972, Selected streamflow characteristics as related to channel geometry of perennial streams in Colorado: U.S. Geological Survey Open-File Report 72-160, 24 p.

Helsel, D.R., and Hirsch, R.M., 2002, Statistical methods in water resources: U.S. Geological Survey Techniques of Water-Resources Investigations, book 4, chap. A3, 510 .

Hortness, J.E., 2006, Estimating low-flow frequency statistics for unregulated streams in Idaho: U.S. Geological Survey Scientific Investigations Report 2006-5035, 31 p.

Hortness, J.E., and Berenbrock, C. 2001, Estimating monthly and annual streamflow statistics at ungaged sites in Idaho: U.S. Geological Survey Water Resources-Investigations Report 2001-4093, 36 p.

Interagency Advisory Committee on Water Data, 1982, Guidelines for determining flood flow frequency: U.S. Geological Survey, Office of Water Data Coordination, Bulletin 17B of the Hydrology Subcommittee, $183 \mathrm{p}$.

Jarrett, R.D., and Costa, J.E. , 1988, Evaluation of the flood hydrology in the Colorado Front Range using precipitation, streamflow and paleoflood data for the Big Thompson River basin: U.S. Geological Survey Water-Resources Investigations Report 87-4117, 37 p.

Jarrett, R.D., and Tomlinson, E.M., 2000, Regional interdisciplinary paleoflood approach to assess extreme flood potential: Water Resources Research, v. 36, no. 10, p. 2957-2984.
Kenney, T.A., Wilkowske, C.D., and Wright, S.J., 2007, Methods for estimating magnitude and frequency of peak flows for natural streams in Utah: U.S. Geological Survey Scientific Investigations Report 2007-5158, 28 p.

Kircher, J.E., Choquette, A.F., and Richter, B.D., 1985, Estimation of natural streamflow characteristics in western Colorado: U.S. Geological Survey Water-Resources Investigations Report 85-4086, 28 p.

Livingston, R.K., and Minges, D.R., 1987, Techniques for estimating regional flood characteristics of small rural watersheds in the plains of eastern Colorado: U.S. Geological Survey Water-Resources Investigations Report 87-4094, $72 \mathrm{p}$.

Lumb, A.M., Kittle, J.L., and Flynn, K.M., 1990, Users manual for ANNIE-A computer program for interactive hydrologic analyses and data management: U.S. Geological Survey Water-Resources Investigations Report 89-4080, $236 \mathrm{p}$.

Matthai, H.F., 1968, Magnitude and frequency of floods in the United States-Part 6B, Missouri River Basin below Sioux City, Iowa: U.S. Geological Survey Water-Supply Paper 1680, $491 \mathrm{p}$.

McCain, J.F., and Jarrett, R.D., 1976, Manual for estimating flood characteristics of natural-flow streams in Colorado: Colorado Water Conservation Board Technical Manual 1, $68 \mathrm{p}$.

Miller, J.F., Frederick, R.H., and Tracy, R. J., 1973, Precipitation frequency Atlas of the United States: National Weather Service, National Oceanic and Atmospheric Administration Atlas 2, Volume 11, accessed August 16, 2007, at http://www.nws.noaa.gov/oh/hdsc/noaaatla2.htm

Miller, K.A., 2003, Peak-flow characteristics of Wyoming streams: U.S. Geological Survey Water-Resources Investigations Report 2003-4107, 79 p.

Montgomery, D.C., Peck, E.A., and Vining, G.G., 2001, Introduction to linear regression analysis-Wiley series in probability and statistics: New York, Wiley, 641 p.

Patterson, J.L., 1964, Magnitude and frequency of floods in the United States-Part 7, Lower Mississippi River Basin: U.S. Geological Survey Water-Supply Paper 1681, 636 p.

Patterson, J.L., 1965, Magnitude and frequency of floods in the United States-Part 8, Western Gulf of Mexico basins: U.S. Geological Survey Water-Supply Paper 1682, 506 p.

Patterson, J.L., and Somers, W.P., 1966, Magnitude and frequency of floods in the United States, Part 9 - Colorado River Basin: U.S. Geological Survey Water-Supply Paper 1683, 475 p. 
Ries, K.G. III, Steeves, P.A,, Coles, J.D., Rea, A.H., and Stewart, D.W., 2004, StreamStats-A U.S. Geological Survey Web application for stream information: U.S. Geological Survey Fact Sheet 2004-3115.

Riggs, H.C., 1972, Low-flow investigations: U.S. Geological Survey Techniques of Water-Resources Investigations, book 4, chap. B1, 18 p.

Robson, S.G., and Banta, E.R., 1995, Ground water atlas of the United States, Segment 2-Arizona, Colorado, New Mexico, Utah: U.S. Geological Survey Hydrologic Investigations Atlas 730-C.

Stedinger, J.R., and Tasker, G.D., 1985, Regional hydrologic analysis I-Ordinary, weighted, and generalized leastsquares compared: American Geophysical Union, Water Resources Research, v. 21, no. 9, p. 1421-1432.

Tasker, G.D., and Stedinger, J.R., 1986, Regional skew with weighted LS regression: American Society of Civil Engineers Journal of Water Resources Planning and Management, v. 112, no. 2, p. 225-237.

Tasker, G.D., and Stedinger, J.R., 1989, An operational GLS model for hydrologic regression: Journal of Hydrology, v. 111 , p. $361-375$.
Thomas, B.E., Hjalmarson, H.W., and Waltemeyer, S.D., 1997, Methods for estimating magnitude and frequency of floods in the southwestern United States: U.S. Geological Survey Water-Supply Paper 2433, 195 p.

U.S. Geological Survey, 2007, National elevation dataset, accessed August 13, 2007, at http://ned.usgs.gov/

U.S. Geological Survey, 2008, National Water Information System-NWISWeb: accessed on various dates in 2008 at http://waterdata.usgs.gov

Vaill, J.E., 1999, Analysis of the magnitude and frequency of floods in Colorado: U.S. Geological Survey WaterResources Investigations Report 99-4190, 35 p.

Waltemeyer, S.D., 2006, Analysis of the magnitude and frequency of peak discharges for the Navajo Nation in Arizona, Utah, Colorado, and New Mexico: U.S. Geological Survey Scientific Investigations Report 2006-5306, 42 p.

Western Regional Climate Center, 2008, Climate of Colorado: Western Regional Climate Center, URL accessed on July 28, 2008 at http://www.wrcc.dri.edu/narratives/ COLORADO.htm 

Supplemental Information 

Table 4. Streamflow-gaging stations used in the development of the regional streamflow regression equations.

[Peak flow, 2-, 5-, 10-, 25-, 50-, 100-, 200-, and 500-year peak flow; Maximum ${ }_{7} Q_{2}$, maximum 7-day, 2-year flow; Maximum ${ }_{7} Q_{10}$, maximum 7-day, 10 -year flow; Maximum ${ }_{7} Q_{50}$, maximum 7-day, 50-year flow; Minimum ${ }_{7} Q_{2}$, minimum 7-day, 2-year flow; Minimum ${ }_{7} Q_{10}$, minimum 7-day, 10-year flow; Minimum ${ }_{7} Q_{50}$, minimum 7-day, 50-year flow; Flow duration, 10-, 25-, 50-, 75-, and 90-percent flow duration; $\mathrm{X}$, station used in the development of regional regression equation for the indicated streamflow characteristic and hydrologic region; --, station not used in the development of regional regression equation for the indicated streamflow characteristic and hydrologic region]

\begin{tabular}{|c|c|c|c|c|c|c|c|c|c|c|c|c|}
\hline \multirow{3}{*}{$\begin{array}{l}\text { Station } \\
\text { number }\end{array}$} & \multirow{3}{*}{ Station name } & \multirow{3}{*}{$\begin{array}{l}\text { Hydrologic } \\
\text { region } \\
\text { (fig. 1) }\end{array}$} & \multicolumn{10}{|c|}{ Streamflow characteristic } \\
\hline & & & \multirow{2}{*}{$\begin{array}{l}\text { Peak } \\
\text { flow }\end{array}$} & \multicolumn{3}{|c|}{ Maximum } & \multicolumn{3}{|c|}{ Minimum } & \multicolumn{2}{|c|}{ Mean flow } & \multirow{2}{*}{$\begin{array}{c}\text { Flow } \\
\text { duration }\end{array}$} \\
\hline & & & & ${ }_{7} Q_{2}$ & ${ }_{7} Q_{10}$ & ${ }_{7} \boldsymbol{O}_{50}$ & ${ }_{7} Q_{2}$ & ${ }_{7} \boldsymbol{Q}_{10}$ & ${ }_{7} \boldsymbol{Q}_{50}$ & Annual & Monthly & \\
\hline 06614800 & Michigan River near Cameron Pass, CO & Mountain & $\mathrm{X}$ & $\mathrm{X}$ & $\mathrm{X}$ & $\mathrm{X}$ & $\mathrm{X}$ & $\mathrm{X}$ & $\mathrm{X}$ & $\mathrm{X}$ & $\mathrm{X}$ & $\mathrm{X}$ \\
\hline 06616000 & North Fork Michigan River near Gould, CO & Mountain & $\mathrm{X}$ & $X$ & $\mathrm{X}$ & $\mathrm{X}$ & $\mathrm{X}$ & $\mathrm{X}$ & -- & $\mathrm{X}$ & $\mathrm{X}$ & $\mathrm{X}$ \\
\hline 06617100 & Michigan River at Walden, CO & Mountain & $\mathrm{X}$ & $\mathrm{X}$ & $\mathrm{X}$ & $\mathrm{X}$ & $\mathrm{X}$ & $\mathrm{X}$ & $\mathrm{X}$ & $\mathrm{X}$ & $\mathrm{X}$ & $\mathrm{X}$ \\
\hline 06618500 & Illinois Creek at Walden, $\mathrm{CO}$ & Mountain & $\mathrm{X}$ & $\mathrm{X}$ & $\mathrm{X}$ & $\mathrm{X}$ & $\mathrm{X}$ & $\mathrm{X}$ & -- & $\mathrm{X}$ & $\mathrm{X}$ & $\mathrm{X}$ \\
\hline 06619000 & Michigan River near Cowdrey, CO & Mountain & $\mathrm{X}$ & $\mathrm{X}$ & $\mathrm{X}$ & $\mathrm{X}$ & $\mathrm{X}$ & -- & -- & $X$ & $\mathrm{X}$ & $\mathrm{X}$ \\
\hline 06620400 & Douglas Creek above Keystone, WY & Mountain & $\mathrm{X}$ & $\mathrm{X}$ & $\mathrm{X}$ & $\mathrm{X}$ & & -- & -- & -- & -- & -- \\
\hline 06621000 & Douglas Creek near Foxpark, WY & Mountain & $\mathrm{X}$ & $\mathrm{X}$ & $\mathrm{X}$ & $\mathrm{X}$ & $\mathrm{X}$ & $\mathrm{X}$ & $\mathrm{X}$ & $\mathrm{X}$ & $\mathrm{X}$ & $\mathrm{X}$ \\
\hline 06622000 & Big Creek at Big Creek Ranger Station, WY & Mountain & $\mathrm{X}$ & -- & -- & - & -- & -- & -- & -- & -- & -- \\
\hline 06622500 & French Creek near French, WY & Mountain & $\mathrm{X}$ & $\mathrm{X}$ & $\mathrm{X}$ & $\mathrm{X}$ & $\mathrm{X}$ & $\mathrm{X}$ & $\mathrm{X}$ & $\mathrm{X}$ & $\mathrm{X}$ & $\mathrm{X}$ \\
\hline 06622700 & North Brush Creek near Saratoga, WY & Mountain & $\mathrm{X}$ & $\mathrm{X}$ & $\mathrm{X}$ & $\mathrm{X}$ & $\mathrm{X}$ & $\mathrm{X}$ & $\mathrm{X}$ & $X$ & $\mathrm{X}$ & $\mathrm{X}$ \\
\hline 06623800 & $\begin{array}{l}\text { Encampment River above Hog Park Creek, near } \\
\text { Encampment, WY }\end{array}$ & Mountain & $\mathrm{X}$ & $\mathrm{X}$ & $\mathrm{X}$ & $\mathrm{X}$ & $\mathrm{X}$ & $\mathrm{X}$ & $\mathrm{X}$ & $\mathrm{X}$ & $\mathrm{X}$ & $\mathrm{X}$ \\
\hline 06624500 & Encampment River at Encampment, WY & Mountain & $\mathrm{X}$ & -- & -- & -- & -- & -- & -- & $\mathrm{X}$ & $\mathrm{X}$ & -- \\
\hline 06627500 & Jack Creek at Matheson Ranch near Saratoga, WY & Northwest & $\mathrm{X}$ & -- & -- & -- & -- & -- & -- & $X$ & $\mathrm{X}$ & -- \\
\hline 06629100 & Rattlesnake Creek near Walcott, WY & Northwest & $\mathrm{X}$ & -- & -- & -- & -- & -- & -- & -- & -- & -- \\
\hline 06630800 & Bear Creek near Elk Mountain, WY & Mountain & $\mathrm{X}$ & -- & -- & -- & -- & -- & -- & -- & -- & -- \\
\hline 06631000 & Medicine Bow River near Medicine Bow, WY & Northwest & $\mathrm{X}$ & -- & -- & -- & -- & -- & -- & $\mathrm{X}$ & $\mathrm{X}$ & -- \\
\hline 06631100 & Wagonhound Creek near Elk Mountain, WY & Mountain & $\mathrm{X}$ & -- & -- & -- & -- & -- & -- & - & -- & -- \\
\hline 06632400 & Rock Creek above King Canyon Canal, near Arlington, WY & Mountain & $\mathrm{X}$ & $\mathrm{X}$ & $\mathrm{X}$ & $\mathrm{X}$ & $\mathrm{X}$ & $\mathrm{X}$ & $\mathrm{X}$ & $\mathrm{X}$ & $\mathrm{X}$ & $\mathrm{X}$ \\
\hline 06696980 & Tarryall Creek at Upper Station near Como, CO & Mountain & $\mathrm{X}$ & $\mathrm{X}$ & $\mathrm{X}$ & $\mathrm{X}$ & -- & -- & -- & $\mathrm{X}$ & $\mathrm{X}$ & $\mathrm{X}$ \\
\hline 06699005 & Tarryall Creek below Rock Creek near Jefferson, CO & Mountain & $\mathrm{X}$ & -- & -- & -- & -- & -- & -- & -- & -- & -- \\
\hline 06700500 & Goose Creek above Cheesman Lake, CO & Mountain & $\mathrm{X}$ & -- & -- & -- & -- & -- & -- & -- & -- & -- \\
\hline 06706000 & $\begin{array}{l}\text { North Fork South Platte River below Geneva Creek, } \\
\text { at Grant, CO }\end{array}$ & Mountain & $\mathrm{X}$ & $\mathrm{X}$ & $\mathrm{X}$ & $\mathrm{X}$ & $\mathrm{X}$ & $\mathrm{X}$ & $\mathrm{X}$ & $\mathrm{X}$ & $\mathrm{X}$ & $\mathrm{X}$ \\
\hline 06707000 & North Fork South Platte River at South Platte, CO & Plains & $\mathrm{X}$ & -- & -- & -- & -- & -- & -- & -- & -- & -- \\
\hline 06709500 & Plum Creek near Louviers, CO & Plains & $\mathrm{X}$ & -- & -- & -- & -- & -- & -- & -- & -- & -- \\
\hline 06710385 & Bear Creek above Evergreen, $\mathrm{CO}$ & Mountain & $\mathrm{X}$ & -- & -- & -- & -- & -- & -- & -- & -- & -- \\
\hline 06710400 & Cub Creek at Evergreen, $\mathrm{CO}$ & Mountain & $\mathrm{X}$ & -- & -- & -- & -- & -- & -- & -- & -- & -- \\
\hline 06710600 & Mount Vernon Creek near Morrison, CO & Plains & $\mathrm{X}$ & -- & -- & -- & -- & -- & -- & -- & -- & -- \\
\hline 06710990 & Parmalee Gulch at Mouth, at Indian Hills, CO & Plains & $\mathrm{X}$ & -- & -- & -- & -- & -- & -- & -- & -- & -- \\
\hline 06711000 & Turkey Creek near Morrison, CO & Plains & $\mathrm{X}$ & -- & -- & -- & -- & -- & -- & -- & -- & -- \\
\hline 06712000 & Cherry Creek near Franktown, CO & Plains & $\mathrm{X}$ & -- & -- & -- & -- & -- & -- & -- & -- & -- \\
\hline 06716500 & Clear Creek near Lawson, CO & Mountain & $\mathrm{X}$ & $\mathrm{X}$ & $\mathrm{X}$ & $\mathrm{X}$ & $\mathrm{X}$ & $\mathrm{X}$ & $\mathrm{X}$ & $\mathrm{X}$ & $\mathrm{X}$ & $\mathrm{X}$ \\
\hline 06717400 & Chicago Creek below Devils Canyon near Idaho Sprgs CO & Mountain & $\mathrm{X}$ & $\mathrm{X}$ & $\mathrm{X}$ & $\mathrm{X}$ & $\mathrm{X}$ & $\mathrm{X}$ & $\mathrm{X}$ & $\mathrm{X}$ & $\mathrm{X}$ & $\mathrm{X}$ \\
\hline 06719500 & Clear Creek near Golden, CO & Plains & $\mathrm{X}$ & -- & -- & -- & -- & -- & -- & - & -- & - \\
\hline 06721500 & North St. Vrain Creek near Allens Park, CO & Mountain & $\mathrm{X}$ & $\mathrm{X}$ & $\mathrm{X}$ & $\mathrm{X}$ & $\mathrm{X}$ & $\mathrm{X}$ & $\mathrm{X}$ & $\mathrm{X}$ & $\mathrm{X}$ & $\mathrm{X}$ \\
\hline 06722500 & South St. Vrain Creek near Ward, CO & Mountain & $\mathrm{X}$ & $\mathrm{X}$ & $\mathrm{X}$ & $\mathrm{X}$ & $\mathrm{X}$ & $\mathrm{X}$ & $\mathrm{X}$ & $\mathrm{X}$ & $\mathrm{X}$ & $\mathrm{X}$ \\
\hline 06723000 & Middle St. Vrain Creek near Allens Park, CO & Mountain & $\mathrm{X}$ & $\mathrm{X}$ & $\mathrm{X}$ & $\mathrm{X}$ & -- & -- & -- & $\mathrm{X}$ & $\mathrm{X}$ & $\mathrm{X}$ \\
\hline 06725500 & Middle Boulder Creek at Nederland, CO & Mountain & $\mathrm{X}$ & $\mathrm{X}$ & $\mathrm{X}$ & $\mathrm{X}$ & $\mathrm{X}$ & $\mathrm{X}$ & $\mathrm{X}$ & $X$ & $\mathrm{X}$ & $\mathrm{X}$ \\
\hline
\end{tabular}


[Peak flow, 2-, 5-, 10-, 25-, 50-, 100-, 200-, and 500-year peak flow; Maximum ${ }_{7} Q_{2}$, maximum 7-day, 2-year flow; Maximum ${ }_{7} Q_{10}$, maximum 7-day, 10 -year flow; Maximum ${ }_{7} Q_{50}$, maximum 7-day, 50-year flow; Minimum ${ }_{7} Q_{2}$, minimum 7-day, 2-year flow; Minimum ${ }_{7} Q_{10}$, minimum 7-day, 10-year flow; Minimum ${ }_{7} Q_{50}$, minimum 7-day, 50-year flow; Flow duration, 10-, 25-, 50-, 75-, and 90-percent flow duration; $\mathrm{X}$, station used in the development of regional regression equation for the indicated streamflow characteristic and hydrologic region; --, station not used in the development of regional regression equation for the indicated streamflow characteristic and hydrologic region]

\begin{tabular}{|c|c|c|c|c|c|c|c|c|c|c|c|c|}
\hline \multirow{3}{*}{$\begin{array}{l}\text { Station } \\
\text { number }\end{array}$} & \multirow{3}{*}{ Station name } & \multirow{3}{*}{$\begin{array}{l}\text { Hydrologic } \\
\text { region } \\
\text { (fig. 1) }\end{array}$} & \multicolumn{10}{|c|}{ Streamflow characteristic } \\
\hline & & & \multirow{2}{*}{$\begin{array}{l}\text { Peak } \\
\text { flow }\end{array}$} & \multicolumn{3}{|c|}{ Maximum } & \multicolumn{3}{|c|}{ Minimum } & \multicolumn{2}{|c|}{ Mean flow } & \multirow{2}{*}{$\begin{array}{c}\text { Flow } \\
\text { duration }\end{array}$} \\
\hline & & & & ${ }_{7} Q_{2}$ & ${ }_{7} Q_{10}$ & ${ }_{7} Q_{50}$ & ${ }_{7} a_{2}$ & ${ }_{7} Q_{10}$ & ${ }_{7} \boldsymbol{Q}_{50}$ & Annual & Monthly & \\
\hline 06726900 & Bummers Gulch near El Vado, CO & Plains & $\mathrm{X}$ & -- & -- & -- & -- & -- & -- & -- & -- & -- \\
\hline 06729000 & South Boulder Creek near Rollinsville, CO & Mountain & $\mathrm{X}$ & $\mathrm{X}$ & $\mathrm{X}$ & $\mathrm{X}$ & $\mathrm{X}$ & $X$ & $\mathrm{X}$ & $\mathrm{X}$ & $\mathrm{X}$ & $\mathrm{X}$ \\
\hline 06730300 & Coal Creek near Plainview, CO & Plains & $X$ & -- & -- & -- & -- & -- & -- & -- & -- & -- \\
\hline 06732000 & Glacier Creek near Estes Park, CO & Mountain & $\mathrm{X}$ & $\mathrm{X}$ & $\mathrm{X}$ & $\mathrm{X}$ & $\mathrm{X}$ & $\mathrm{X}$ & $\mathrm{X}$ & $\mathrm{X}$ & $\mathrm{X}$ & $\mathrm{X}$ \\
\hline 06739500 & Buckhorn Creek near Masonville, CO & Plains & $\mathrm{X}$ & -- & - & - & - & - & - & - & - & -- \\
\hline 06748200 & Fall Creek near Rustic, CO & Mountain & $\mathrm{X}$ & $\mathrm{X}$ & $X$ & $\mathrm{X}$ & $X$ & $X$ & $\mathrm{X}$ & $X$ & $\mathrm{X}$ & $\mathrm{X}$ \\
\hline 06748510 & Little Beaver Creek near Idylwilde, CO & Mountain & $\mathrm{X}$ & $\mathrm{X}$ & $X$ & $\mathrm{X}$ & $X$ & $\mathrm{X}$ & $X$ & $X$ & $\mathrm{X}$ & $\mathrm{X}$ \\
\hline 06748530 & Little Beaver Creek near Rustic, CO & Mountain & $\mathrm{X}$ & $\mathrm{X}$ & $\mathrm{X}$ & $\mathrm{X}$ & $\mathrm{X}$ & $\mathrm{X}$ & $\mathrm{X}$ & $\mathrm{X}$ & $\mathrm{X}$ & $\mathrm{X}$ \\
\hline 06748600 & South Fork Cache La Poudre River near Rustic, CO & Mountain & $\mathrm{X}$ & $\mathrm{X}$ & $\mathrm{X}$ & $\mathrm{X}$ & $\mathrm{X}$ & $\mathrm{X}$ & $\mathrm{X}$ & $\mathrm{X}$ & $\mathrm{X}$ & $\mathrm{X}$ \\
\hline 06755000 & South Crow Creek near Hecla, WY & Plains & $\mathrm{X}$ & -- & -- & -- & -- & -- & -- & $\mathrm{X}$ & $\mathrm{X}$ & -- \\
\hline 06758200 & Kiowa Creek at Kiowa, CO & Plains & $\mathrm{X}$ & -- & -- & -- & -- & -- & -- & -- & -- & -- \\
\hline 06758250 & Kiowa Creek Tributary near Bennett, CO & Plains & $\mathrm{X}$ & -- & -- & -- & -- & -- & -- & -- & -- & -- \\
\hline 06758700 & Middle Bijou Creek Tributary near Deer Trail, CO & Plains & $X$ & -- & -- & -- & -- & -- & -- & -- & -- & -- \\
\hline 06761900 & Lodgepole Creek Tributary near Pine Bluffs, WY & Plains & $X$ & -- & -- & -- & -- & -- & -- & -- & -- & -- \\
\hline 06762500 & Lodgepole Creek at Bushnell, Nebr. & Plains & $\mathrm{X}$ & -- & -- & -- & -- & -- & -- & -- & -- & -- \\
\hline 06762600 & Lodgepole Creek Tributary No. 2 near Albin, WY & Plains & $\mathrm{X}$ & -- & -- & -- & -- & -- & -- & -- & -- & -- \\
\hline 06821300 & North Fork Arikaree River Tributary near Shaw, CO & Plains & $\mathrm{X}$ & -- & -- & -- & -- & -- & -- & -- & -- & -- \\
\hline 06821400 & North Fork Black Wolf Creek near Vernon, CO & Plains & $\mathrm{X}$ & -- & -- & -- & -- & -- & -- & -- & -- & -- \\
\hline 06823500 & Buffalo Creek near Haigler, NE & Plains & $\mathrm{X}$ & -- & -- & -- & -- & -- & -- & -- & -- & -- \\
\hline 06825500 & Landsman Creek near Hale, $\mathrm{CO}$ & Plains & $\mathrm{X}$ & -- & -- & -- & -- & -- & -- & -- & -- & -- \\
\hline 06844700 & South Fork Sappa Creek near Brewster, KS & Plains & $\mathrm{X}$ & -- & -- & -- & -- & -- & -- & -- & -- & -- \\
\hline 06844800 & South Fork Sappa Creek Tributary near Goodland, KS & Plains & $\mathrm{X}$ & -- & -- & -- & -- & -- & -- & -- & -- & -- \\
\hline 06858500 & North Fork Smoky Hill River near Mcallaster, KS & Plains & $\mathrm{X}$ & -- & -- & -- & -- & -- & -- & -- & -- & -- \\
\hline 07079500 & East Fork Arkansas River near Leadville, CO & Mountain & $\mathrm{X}$ & $\mathrm{X}$ & $\mathrm{X}$ & $\mathrm{X}$ & $\mathrm{X}$ & $\mathrm{X}$ & $\mathrm{X}$ & $\mathrm{X}$ & $\mathrm{X}$ & $\mathrm{X}$ \\
\hline 07081000 & Tennessee Creek near Leadville, CO & Mountain & $X$ & $\mathrm{X}$ & $\mathrm{X}$ & $\mathrm{X}$ & $\mathrm{X}$ & $\mathrm{X}$ & $\mathrm{X}$ & $X$ & $\mathrm{X}$ & $\mathrm{X}$ \\
\hline 07082000 & Lake Fork above Sugar Loaf Reservoir, CO & Mountain & $\mathrm{X}$ & $\mathrm{X}$ & $\mathrm{X}$ & $\mathrm{X}$ & $\mathrm{X}$ & $\mathrm{X}$ & $\mathrm{X}$ & $\mathrm{X}$ & $\mathrm{X}$ & $\mathrm{X}$ \\
\hline 07083000 & Halfmoon Creek near Malta, CO & Mountain & $\mathrm{X}$ & $\mathrm{X}$ & $\mathrm{X}$ & $\mathrm{X}$ & $\mathrm{X}$ & $\mathrm{X}$ & $\mathrm{X}$ & $\mathrm{X}$ & $\mathrm{X}$ & $\mathrm{X}$ \\
\hline 07089000 & Cottonwood C below Hot Springs, near Buena Vista, CO & Mountain & $\mathrm{X}$ & -- & - & -- & - & -- & - & -- & -- & -- \\
\hline 07091000 & Chalk Creek near Nathrop, CO & Mountain & $\mathrm{X}$ & -- & -- & -- & -- & -- & -- & -- & -- & -- \\
\hline 07093500 & South Arkansas River near Salida, CO & Mountain & $\mathrm{X}$ & -- & -- & -- & -- & -- & -- & -- & -- & -- \\
\hline 07093775 & Badger Creek, Lower Station, near Howard, CO & Plains & $\mathrm{X}$ & -- & -- & -- & -- & -- & -- & -- & -- & -- \\
\hline 07095000 & Grape Creek near Westcliffe, CO & Plains & $\mathrm{X}$ & -- & -- & -- & -- & -- & -- & -- & -- & -- \\
\hline 07096500 & Fourmile Creek near Canon City, CO & Plains & $\mathrm{X}$ & -- & -- & -- & -- & -- & -- & -- & -- & -- \\
\hline 07099100 & Beaver Creek near Portland, CO & Plains & $\mathrm{X}$ & -- & -- & -- & -- & -- & -- & -- & -- & -- \\
\hline 07099230 & Turkey Creek above Teller Reservoir near Stone City, CO & Plains & $\mathrm{X}$ & -- & -- & -- & -- & -- & -- & -- & -- & -- \\
\hline 07105920 & $\begin{array}{l}\text { Little Fountain Creek above Keaton Reservoir, near } \\
\text { Fort Carson, CO }\end{array}$ & Plains & $\mathrm{X}$ & -- & -- & -- & -- & -- & -- & -- & -- & -- \\
\hline 07105945 & Rock Creek above Fort Carson Reservation, CO & Plains & $\mathrm{X}$ & -- & -- & -- & -- & -- & -- & -- & -- & -- \\
\hline 07107500 & St. Charles River at Burnt Mill, CO & Plains & $\mathrm{X}$ & -- & -- & -- & -- & -- & -- & -- & -- & -- \\
\hline
\end{tabular}


Table 4. Streamflow-gaging stations used in the development of the regional streamflow regression equations.-Continued

[Peak flow, 2-, 5-, 10-, 25-, 50-, 100-, 200-, and 500-year peak flow; Maximum ${ }_{7} Q_{2}$, maximum 7-day, 2-year flow; Maximum ${ }_{7} Q_{10}$, maximum 7-day, 10 -year flow; Maximum ${ }_{7} Q_{50}$, maximum 7-day, 50-year flow; Minimum ${ }_{7} Q_{2}$, minimum 7-day, 2-year flow; Minimum ${ }_{7} Q_{10}$, minimum 7-day, 10-year flow; Minimum ${ }_{7} Q_{50}$, minimum 7-day, 50-year flow; Flow duration, 10 -, 25-, 50-, 75 -, and 90-percent flow duration; $\mathrm{X}$, station used in the development of regional regression equation for the indicated streamflow characteristic and hydrologic region; --, station not used in the development of regional regression equation for the indicated streamflow characteristic and hydrologic region]

\begin{tabular}{|c|c|c|c|c|c|c|c|c|c|c|c|c|}
\hline \multirow{3}{*}{$\begin{array}{l}\text { Station } \\
\text { number }\end{array}$} & \multirow{3}{*}{ Station name } & \multirow{3}{*}{$\begin{array}{l}\text { Hydrologic } \\
\text { region } \\
\text { (fig. 1) }\end{array}$} & \multicolumn{10}{|c|}{ Streamflow characteristic } \\
\hline & & & \multirow{2}{*}{$\begin{array}{l}\text { Peak } \\
\text { flow }\end{array}$} & \multicolumn{3}{|c|}{ Maximum } & \multicolumn{3}{|c|}{ Minimum } & \multicolumn{2}{|c|}{ Mean flow } & \multirow{2}{*}{$\begin{array}{c}\text { Flow } \\
\text { duration }\end{array}$} \\
\hline & & & & ${ }_{7} \boldsymbol{Q}_{2}$ & ${ }_{7} Q_{10}$ & ${ }_{7} \boldsymbol{Q}_{50}$ & ${ }_{7} a_{2}$ & ${ }_{7} Q_{10}$ & ${ }_{7} Q_{50}$ & Annual & Monthly & \\
\hline 07114000 & Cucharas River at Boyd Ranch, near La Veta, CO & RioGrande & $\mathrm{X}$ & -- & -- & -- & -- & -- & -- & -- & -- & -- \\
\hline 07118000 & Apishapa River near Aguilar, CO & Plains & $\mathrm{X}$ & -- & -- & -- & -- & -- & -- & -- & -- & -- \\
\hline 07120620 & Big Arroyo near Thatcher, CO & Plains & $\mathrm{X}$ & -- & -- & -- & -- & -- & -- & -- & -- & -- \\
\hline 07121500 & Timpas Creek at Mouth near Swink, CO & Plains & $\mathrm{X}$ & -- & -- & -- & -- & -- & -- & -- & -- & -- \\
\hline 07124300 & Long Canyon Creek near Madrid, CO & Plains & $\mathrm{X}$ & -- & -- & -- & -- & -- & -- & -- & -- & -- \\
\hline 07125100 & Frijole Creek near Alfalfa, $\mathrm{CO}$ & Plains & $\mathrm{X}$ & -- & -- & -- & -- & -- & -- & -- & -- & -- \\
\hline 07125500 & San Francisco Creek near Alfalfa, CO & Plains & $\mathrm{X}$ & -- & -- & -- & -- & -- & -- & -- & -- & -- \\
\hline 07126100 & Luning Arroyo near Model, CO & Plains & $\mathrm{X}$ & -- & -- & -- & -- & -- & -- & -- & -- & -- \\
\hline 07126200 & Van Bremer Arroyo near Model, CO & Plains & $\mathrm{X}$ & -- & -- & -- & -- & -- & -- & -- & -- & -- \\
\hline 07126325 & Taylor Arroyo below Rock Crossing, near Thatcher, CO & Plains & $\mathrm{X}$ & -- & -- & -- & -- & -- & -- & -- & -- & -- \\
\hline 07126390 & Lockwood Canyon Creek near Thatcher, CO & Plains & $\mathrm{X}$ & -- & -- & -- & -- & -- & -- & -- & -- & -- \\
\hline 07126415 & Red Rock Canyon Creek at Mouth near Thatcher, CO & Plains & $\mathrm{X}$ & -- & -- & -- & -- & -- & -- & -- & -- & -- \\
\hline 07126470 & Chacuaco Creek at Mouth near Timpas, CO & Plains & $\mathrm{X}$ & -- & -- & -- & -- & -- & -- & -- & -- & -- \\
\hline 07126480 & Bent Canyon Creek at Mouth near Timpas, CO & Plains & $\mathrm{X}$ & -- & -- & -- & -- & -- & -- & -- & -- & -- \\
\hline 07133200 & Clay Creek Tributary near Deora, CO & Plains & $\mathrm{X}$ & -- & -- & -- & -- & -- & -- & -- & -- & -- \\
\hline 07138600 & White Woman C Tributary near Selkirk, KS & Plains & $\mathrm{X}$ & -- & -- & -- & -- & -- & -- & -- & -- & -- \\
\hline 07138650 & White Woman C near Leoti, KS & Plains & $X$ & -- & -- & -- & -- & -- & -- & -- & -- & -- \\
\hline 07153500 & Dry Cimarron R near Guy, NM & Plains & $\mathrm{X}$ & -- & -- & -- & -- & -- & -- & -- & -- & -- \\
\hline 07154400 & Carrizozo Creek near Kenton, OK & Plains & $\mathrm{X}$ & -- & -- & -- & -- & -- & -- & -- & -- & -- \\
\hline 07154650 & Tesesquite Creek near Kenton, OK & Plains & $\mathrm{X}$ & -- & -- & -- & -- & -- & -- & -- & -- & -- \\
\hline 07155100 & Cpld Springs Creek near Wheeless, OK & Plains & $\mathrm{X}$ & -- & -- & -- & -- & -- & -- & -- & -- & -- \\
\hline 07155590 & Cimarron River near Elkhart, KS & Plains & $\mathrm{X}$ & -- & -- & -- & -- & -- & -- & -- & -- & -- \\
\hline 07155900 & North Fork Cimarron River Tributary near Elkhart, KS & Plains & $\mathrm{X}$ & -- & -- & -- & -- & -- & -- & -- & -- & -- \\
\hline 07156010 & North Fork Cimarron River at Richfield, KS & Plains & $\mathrm{X}$ & -- & -- & -- & -- & -- & -- & -- & -- & -- \\
\hline 07156100 & Sand Arroyo Creek near Johnson, KS & Plains & $\mathrm{X}$ & -- & -- & -- & -- & -- & -- & -- & -- & -- \\
\hline 07156220 & Bear Creek near Johnson, KS & Plains & $X$ & -- & -- & -- & -- & -- & -- & -- & -- & -- \\
\hline 07156600 & Cimarron River Tributary near Moscow, KS & Plains & $\mathrm{X}$ & -- & -- & -- & -- & -- & -- & -- & -- & -- \\
\hline 07199000 & Canadian River near Hebron, NM & Plains & $\mathrm{X}$ & -- & -- & -- & -- & -- & -- & -- & -- & -- \\
\hline 07201000 & Raton Creek at Raton, NM & Plains & $\mathrm{X}$ & -- & -- & -- & -- & -- & -- & -- & -- & -- \\
\hline 07201200 & Chicorica Cr Tributary near Raton, NM & Plains & $\mathrm{X}$ & -- & -- & -- & -- & -- & -- & -- & -- & -- \\
\hline 07203000 & Vermejo River near Dawson, NM & Plains & $\mathrm{X}$ & -- & -- & -- & -- & -- & -- & -- & -- & -- \\
\hline 07204000 & Moreno Creek at Eagle Nest, NM & RioGrande & $\mathrm{X}$ & -- & -- & -- & -- & -- & -- & -- & -- & -- \\
\hline 07204500 & Cieneguilla Cr near Eagle Nest, NM & RioGrande & $\mathrm{X}$ & -- & -- & -- & -- & -- & -- & -- & -- & -- \\
\hline 07205000 & Sixmile Creek near Eagle Nest, NM & RioGrande & $\mathrm{X}$ & -- & -- & -- & -- & -- & -- & -- & -- & -- \\
\hline 07206400 & Clear C near Ute Park, NM & RioGrande & $\mathrm{X}$ & -- & -- & -- & -- & -- & -- & -- & -- & -- \\
\hline 07207500 & Ponil Creek near Cimarron, NM & Plains & $\mathrm{X}$ & -- & -- & -- & -- & -- & -- & -- & -- & -- \\
\hline 07208500 & Rayado Creek near Cimarron, NM & Plains & $\mathrm{X}$ & -- & -- & -- & -- & -- & -- & -- & -- & -- \\
\hline 07211000 & Cimarron River at Springer, NM & Plains & $\mathrm{X}$ & -- & -- & -- & -- & -- & -- & -- & -- & -- \\
\hline 07225500 & Ute Creek near Gladstone, NM & Plains & $\mathrm{X}$ & -- & -- & -- & -- & -- & -- & -- & -- & -- \\
\hline
\end{tabular}


[Peak flow, 2-, 5-, 10-, 25-, 50-, 100-, 200-, and 500-year peak flow; Maximum ${ }_{7} Q_{2}$, maximum 7-day, 2-year flow; Maximum ${ }_{7} Q_{10}$, maximum 7-day, 10 -year flow; Maximum ${ }_{7} Q_{50}$, maximum 7-day, 50-year flow; Minimum ${ }_{7} Q_{2}$, minimum 7-day, 2-year flow; Minimum ${ }_{7} Q_{10}$, minimum 7-day, 10-year flow; Minimum ${ }_{7} Q_{50}$, minimum 7-day, 50-year flow; Flow duration, 10-, 25-, 50-, 75-, and 90-percent flow duration; $\mathrm{X}$, station used in the development of regional regression equation for the indicated streamflow characteristic and hydrologic region; --, station not used in the development of regional regression equation for the indicated streamflow characteristic and hydrologic region]

\begin{tabular}{|c|c|c|c|c|c|c|c|c|c|c|c|c|}
\hline \multirow{3}{*}{$\begin{array}{l}\text { Station } \\
\text { number }\end{array}$} & \multirow{3}{*}{ Station name } & \multirow{3}{*}{$\begin{array}{l}\text { Hydrologic } \\
\text { region } \\
\text { (fig. 1) }\end{array}$} & \multicolumn{10}{|c|}{ Streamflow characteristic } \\
\hline & & & \multirow{2}{*}{$\begin{array}{l}\text { Peak } \\
\text { flow }\end{array}$} & \multicolumn{3}{|c|}{ Maximum } & \multicolumn{3}{|c|}{ Minimum } & \multicolumn{2}{|c|}{ Mean flow } & \multirow{2}{*}{$\begin{array}{c}\text { Flow } \\
\text { duration }\end{array}$} \\
\hline & & & & ${ }_{7} Q_{2}$ & ${ }_{7} Q_{10}$ & ${ }_{7} \boldsymbol{Q}_{50}$ & ${ }_{7} a_{2}$ & ${ }_{7} Q_{10}$ & ${ }_{7} \boldsymbol{Q}_{50}$ & Annual & Monthly & \\
\hline 07227295 & Sandy Arroyo Tributary near Clayton, NM & Plains & $\mathrm{X}$ & -- & -- & -- & -- & -- & -- & -- & -- & -- \\
\hline 07232500 & Beaver River near Guymon, OK & Plains & $\mathrm{X}$ & -- & -- & -- & -- & -- & -- & -- & -- & -- \\
\hline 07232650 & Aqua Frio Creek near Felt, OK & Plains & $\mathrm{X}$ & -- & -- & -- & -- & -- & -- & -- & -- & -- \\
\hline 08216500 & Willow Creek at Creede, $\mathrm{CO}$ & RioGrande & $\mathrm{X}$ & $\mathrm{X}$ & $\mathrm{X}$ & $\mathrm{X}$ & $\mathrm{X}$ & $\mathrm{X}$ & $\mathrm{X}$ & $\mathrm{X}$ & $\mathrm{X}$ & $\mathrm{X}$ \\
\hline 08218500 & Goose Creek at Wagonwheel Gap, CO & RioGrande & $\mathrm{X}$ & $\mathrm{X}$ & $X$ & $\mathrm{X}$ & $X$ & $\mathrm{X}$ & $\mathrm{X}$ & $X$ & $\mathrm{X}$ & $\mathrm{X}$ \\
\hline 08219500 & South Fork Rio Grande at South Fork, CO & RioGrande & $\mathrm{X}$ & $\mathrm{X}$ & $\mathrm{X}$ & $\mathrm{X}$ & $\mathrm{X}$ & $\mathrm{X}$ & $\mathrm{X}$ & $\mathrm{X}$ & $\mathrm{X}$ & $\mathrm{X}$ \\
\hline 08220500 & Pinos Creek near Del Norte, $\mathrm{CO}$ & RioGrande & $\mathrm{X}$ & $\mathrm{X}$ & $\mathrm{X}$ & $\mathrm{X}$ & $\mathrm{X}$ & $\mathrm{X}$ & $\mathrm{X}$ & $\mathrm{X}$ & $\mathrm{X}$ & $\mathrm{X}$ \\
\hline 08223500 & Rock Creek near Monte Vista, CO & RioGrande & $\mathrm{X}$ & $\mathrm{X}$ & $\mathrm{X}$ & $\mathrm{X}$ & $\mathrm{X}$ & $\mathrm{X}$ & $\mathrm{X}$ & $\mathrm{X}$ & $\mathrm{X}$ & $\mathrm{X}$ \\
\hline 08224500 & Kerber Cr above Little Kerber Cr near Villa Grove, CO & RioGrande & $\mathrm{X}$ & $\mathrm{X}$ & $\mathrm{X}$ & $\mathrm{X}$ & $\mathrm{X}$ & $\mathrm{X}$ & - & $\mathrm{X}$ & $\mathrm{X}$ & $\mathrm{X}$ \\
\hline 08227000 & Saguache Creek near Saguache, CO & RioGrande & $\mathrm{X}$ & $\mathrm{X}$ & $\mathrm{X}$ & $\mathrm{X}$ & $\mathrm{X}$ & $\mathrm{X}$ & $\mathrm{X}$ & $\mathrm{X}$ & $\mathrm{X}$ & $\mathrm{X}$ \\
\hline 08227500 & North Crestone Creek near Crestone, $\mathrm{CO}$ & RioGrande & $\mathrm{X}$ & $\mathrm{X}$ & $\mathrm{X}$ & $\mathrm{X}$ & $\mathrm{X}$ & $\mathrm{X}$ & $\mathrm{X}$ & $\mathrm{X}$ & $\mathrm{X}$ & $\mathrm{X}$ \\
\hline 08230500 & Carnero Creek near La Garita, CO & RioGrande & $\mathrm{X}$ & $\mathrm{X}$ & $\mathrm{X}$ & $\mathrm{X}$ & $\mathrm{X}$ & $X$ & -- & $\mathrm{X}$ & $\mathrm{X}$ & $\mathrm{X}$ \\
\hline 08231000 & La Garita Creek near La Garita, CO & RioGrande & $\mathrm{X}$ & $X$ & $\mathrm{X}$ & $\mathrm{X}$ & $\mathrm{X}$ & $\mathrm{X}$ & $\mathrm{X}$ & $\mathrm{X}$ & $\mathrm{X}$ & $\mathrm{X}$ \\
\hline 08236000 & Alamosa River above Terrace Reservoir, $\mathrm{CO}$ & RioGrande & $\mathrm{X}$ & $\mathrm{X}$ & $\mathrm{X}$ & $\mathrm{X}$ & $\mathrm{X}$ & $\mathrm{X}$ & $\mathrm{X}$ & $\mathrm{X}$ & $\mathrm{X}$ & $\mathrm{X}$ \\
\hline 08240500 & Trinchera $\mathrm{C}$ above Turners Ranch, Nr Ft Garland, $\mathrm{CO}$ & RioGrande & $\mathrm{X}$ & $\mathrm{X}$ & $\mathrm{X}$ & $\mathrm{X}$ & $X$ & $\mathrm{X}$ & $\mathrm{X}$ & $\mathrm{X}$ & $\mathrm{X}$ & $\mathrm{X}$ \\
\hline 08241500 & Sangre De Cristo Creek near Fort Garland, CO & RioGrande & $\mathrm{X}$ & $\mathrm{X}$ & $\mathrm{X}$ & $\mathrm{X}$ & $\mathrm{X}$ & -- & -- & $\mathrm{X}$ & $\mathrm{X}$ & $\mathrm{X}$ \\
\hline 08242500 & Ute Creek near Fort Garland, CO & RioGrande & $\mathrm{X}$ & $\mathrm{X}$ & $X$ & $\mathrm{X}$ & $X$ & $\mathrm{X}$ & -- & $\mathrm{X}$ & $\mathrm{X}$ & $\mathrm{X}$ \\
\hline 08245500 & Conejos River at Platoro, $\mathrm{CO}$ & RioGrande & $\mathrm{X}$ & $\mathrm{X}$ & $\mathrm{X}$ & $\mathrm{X}$ & $\mathrm{X}$ & $\mathrm{X}$ & $\mathrm{X}$ & $\mathrm{X}$ & $\mathrm{X}$ & $\mathrm{X}$ \\
\hline 08246500 & Conejos River near Mogote, $\mathrm{CO}$ & RioGrande & $\mathrm{X}$ & $\mathrm{X}$ & $\mathrm{X}$ & $\mathrm{X}$ & $\mathrm{X}$ & $\mathrm{X}$ & $\mathrm{X}$ & $\mathrm{X}$ & $\mathrm{X}$ & $\mathrm{X}$ \\
\hline 08247500 & San Antonio River at Ortiz, CO & RioGrande & $\mathrm{X}$ & $\mathrm{X}$ & $\mathrm{X}$ & $\mathrm{X}$ & -- & -- & -- & $\mathrm{X}$ & $\mathrm{X}$ & $\mathrm{X}$ \\
\hline 08248000 & Los Pinos River near Ortiz, CO & RioGrande & $\mathrm{X}$ & $\mathrm{X}$ & $\mathrm{X}$ & $\mathrm{X}$ & $\mathrm{X}$ & $\mathrm{X}$ & $\mathrm{X}$ & $\mathrm{X}$ & $\mathrm{X}$ & $\mathrm{X}$ \\
\hline 08248500 & San Antonio River at Mouth, near Manassa, CO & RioGrande & $\mathrm{X}$ & $\mathrm{X}$ & $\mathrm{X}$ & $\mathrm{X}$ & -- & -- & -- & $\mathrm{X}$ & $\mathrm{X}$ & $\mathrm{X}$ \\
\hline 08252500 & Costilla Creek above Costilla Dam, NM & RioGrande & $\mathrm{X}$ & -- & -- & -- & -- & -- & -- & -- & $\mathrm{X}$ & -- \\
\hline 08253000 & Casias Creek near Costilla, NM & RioGrande & $\mathrm{X}$ & -- & -- & -- & -- & -- & -- & -- & $\mathrm{X}$ & -- \\
\hline 08253500 & Santistevan Creek near Costilla, NM & RioGrande & $\mathrm{X}$ & -- & -- & -- & -- & -- & -- & -- & $\mathrm{X}$ & -- \\
\hline 08255000 & Ute Creek near Amalia, NM & RioGrande & $\mathrm{X}$ & -- & -- & -- & -- & -- & -- & -- & $\mathrm{X}$ & -- \\
\hline 08263000 & Latir Creek near Cerro, NM & RioGrande & $\mathrm{X}$ & -- & -- & -- & -- & -- & -- & $\mathrm{X}$ & $\mathrm{X}$ & $\mathrm{X}$ \\
\hline 08264000 & Red River near Red River, NM & RioGrande & $\mathrm{X}$ & $\mathrm{X}$ & $\mathrm{X}$ & $\mathrm{X}$ & $\mathrm{X}$ & $\mathrm{X}$ & $\mathrm{X}$ & $\mathrm{X}$ & $\mathrm{X}$ & $\mathrm{X}$ \\
\hline 08265000 & Red River near Questa, NM & RioGrande & $\mathrm{X}$ & $\mathrm{X}$ & $\mathrm{X}$ & $\mathrm{X}$ & $\mathrm{X}$ & $\mathrm{X}$ & $\mathrm{X}$ & $\mathrm{X}$ & $\mathrm{X}$ & $\mathrm{X}$ \\
\hline 08267500 & Rio Hondo near Valdez, NM & RioGrande & $\mathrm{X}$ & $\mathrm{X}$ & $X$ & $\mathrm{X}$ & $X$ & $\mathrm{X}$ & $\mathrm{X}$ & $X$ & $\mathrm{X}$ & $\mathrm{X}$ \\
\hline 08268500 & Arroyo Hondo at Arroyo Hondo, NM & RioGrande & $\mathrm{X}$ & $\mathrm{X}$ & $\mathrm{X}$ & $\mathrm{X}$ & $\mathrm{X}$ & $\mathrm{X}$ & $\mathrm{X}$ & $\mathrm{X}$ & $\mathrm{X}$ & $\mathrm{X}$ \\
\hline 08269000 & Rio Pueblo De Taos near Taos, NM & RioGrande & $\mathrm{X}$ & $\mathrm{X}$ & $\mathrm{X}$ & $\mathrm{X}$ & $\mathrm{X}$ & $\mathrm{X}$ & $\mathrm{X}$ & $\mathrm{X}$ & $\mathrm{X}$ & $\mathrm{X}$ \\
\hline 08275000 & Rio Fernando De Taos near Taos, NM & RioGrande & $\mathrm{X}$ & $\mathrm{X}$ & $\mathrm{X}$ & $\mathrm{X}$ & $\mathrm{X}$ & $\mathrm{X}$ & $\mathrm{X}$ & $\mathrm{X}$ & $\mathrm{X}$ & $\mathrm{X}$ \\
\hline 08275500 & Rio Grande Del Rancho near Talpa, NM & RioGrande & $\mathrm{X}$ & $\mathrm{X}$ & $X$ & $\mathrm{X}$ & $\mathrm{X}$ & $\mathrm{X}$ & $\mathrm{X}$ & $\mathrm{X}$ & $\mathrm{X}$ & $\mathrm{X}$ \\
\hline 08275600 & Rio Chiquito near Talpa, NM & RioGrande & $\mathrm{X}$ & $\mathrm{X}$ & $\mathrm{X}$ & $\mathrm{X}$ & $\mathrm{X}$ & $\mathrm{X}$ & $\mathrm{X}$ & $\mathrm{X}$ & $\mathrm{X}$ & $\mathrm{X}$ \\
\hline 08281200 & Wolf Creek near Chama, NM & RioGrande & $\mathrm{X}$ & -- & -- & -- & -- & -- & -- & -- & -- & -- \\
\hline 08284000 & Rito De Tierra Amarilla at Tierra Amar, NM & RioGrande & $\mathrm{X}$ & -- & -- & -- & -- & -- & -- & -- & -- & -- \\
\hline 08284100 & Rio Chama near La Puente, NM & RioGrande & $\mathrm{X}$ & $\mathrm{X}$ & $\mathrm{X}$ & $X$ & $\mathrm{X}$ & $\mathrm{X}$ & $\mathrm{X}$ & $\mathrm{X}$ & $\mathrm{X}$ & $\mathrm{X}$ \\
\hline 08284500 & Willow Creek near Park View, NM & RioGrande & $\mathrm{X}$ & $\mathrm{X}$ & $\mathrm{X}$ & $\mathrm{X}$ & -- & - & -- & $\mathrm{X}$ & $\mathrm{X}$ & $\mathrm{X}$ \\
\hline
\end{tabular}


Table 4. Streamflow-gaging stations used in the development of the regional streamflow regression equations.-Continued

[Peak flow, 2-, 5-, 10-, 25-, 50-, 100-, 200-, and 500-year peak flow; Maximum ${ }_{7} Q_{2}$, maximum 7-day, 2-year flow; Maximum ${ }_{7} Q_{10}$, maximum 7-day, 10 -year flow; Maximum ${ }_{7} Q_{50}$, maximum 7-day, 50-year flow; Minimum ${ }_{7} Q_{2}$, minimum 7-day, 2-year flow; Minimum ${ }_{7} Q_{10}$, minimum 7-day, 10-year flow; Minimum ${ }_{7} Q_{50}$, minimum 7-day, 50-year flow; Flow duration, 10-, 25-, 50-, 75-, and 90-percent flow duration; $\mathrm{X}$, station used in the development of regional regression equation for the indicated streamflow characteristic and hydrologic region; --, station not used in the development of regional regression equation for the indicated streamflow characteristic and hydrologic region]

\begin{tabular}{|c|c|c|c|c|c|c|c|c|c|c|c|c|}
\hline \multirow{3}{*}{$\begin{array}{l}\text { Station } \\
\text { number }\end{array}$} & \multirow{3}{*}{ Station name } & \multirow{3}{*}{$\begin{array}{c}\text { Hydrologic } \\
\text { region } \\
\text { (fig. 1) }\end{array}$} & \multicolumn{10}{|c|}{ Streamflow characteristic } \\
\hline & & & \multirow{2}{*}{$\begin{array}{l}\text { Peak } \\
\text { flow }\end{array}$} & \multicolumn{3}{|c|}{ Maximum } & \multicolumn{3}{|c|}{ Minimum } & \multicolumn{2}{|c|}{ Mean flow } & \multirow{2}{*}{$\begin{array}{c}\text { Flow } \\
\text { duration }\end{array}$} \\
\hline & & & & ${ }_{7} O_{2}$ & ${ }_{7} Q_{10}$ & ${ }_{7} Q_{50}$ & ${ }_{7} \boldsymbol{Q}_{2}$ & ${ }_{7} Q_{10}$ & ${ }_{7} Q_{50}$ & Annual & Monthly & \\
\hline 08286650 & Canjilon Creek above Abiquiu Reservoir, NM & RioGrande & $\mathrm{X}$ & -- & -- & -- & -- & -- & -- & -- & -- & -- \\
\hline 08288000 & El Rito near El Rito, NM & RioGrande & $\mathrm{X}$ & $\mathrm{X}$ & $\mathrm{X}$ & $\mathrm{X}$ & $\mathrm{X}$ & $\mathrm{X}$ & $\mathrm{X}$ & $\mathrm{X}$ & $\mathrm{X}$ & $\mathrm{X}$ \\
\hline 08289000 & Rio Ojo Caliente at La Madera, NM & RioGrande & $\mathrm{X}$ & $\mathrm{X}$ & $\mathrm{X}$ & $\mathrm{X}$ & $\mathrm{X}$ & $\mathrm{X}$ & $\mathrm{X}$ & $\mathrm{X}$ & $\mathrm{X}$ & $\mathrm{X}$ \\
\hline 09012500 & North Inlet at Grand Lake, CO & Mountain & $\mathrm{X}$ & $\mathrm{X}$ & $\mathrm{X}$ & $\mathrm{X}$ & $\mathrm{X}$ & $\mathrm{X}$ & $\mathrm{X}$ & $\mathrm{X}$ & $\mathrm{X}$ & $\mathrm{X}$ \\
\hline 09016500 & Arapaho Creek at Monarch Lake Outlet, CO & Mountain & $\mathrm{X}$ & $\mathrm{X}$ & $\mathrm{X}$ & $\mathrm{X}$ & $\mathrm{X}$ & $\mathrm{X}$ & $\mathrm{X}$ & $\mathrm{X}$ & $\mathrm{X}$ & $\mathrm{X}$ \\
\hline 09020000 & Willow Creek near Granby, CO & Mountain & $\mathrm{X}$ & $\mathrm{X}$ & $\mathrm{X}$ & $\mathrm{X}$ & -- & -- & -- & $\mathrm{X}$ & $\mathrm{X}$ & $\mathrm{X}$ \\
\hline 09024000 & Fraser River at Winter Park, CO & Mountain & $\mathrm{X}$ & $\mathrm{X}$ & $\mathrm{X}$ & $\mathrm{X}$ & $\mathrm{X}$ & $\mathrm{X}$ & $X$ & $\mathrm{X}$ & $\mathrm{X}$ & $\mathrm{X}$ \\
\hline 09026500 & St. Louis Creek near Fraser, CO & Mountain & $X$ & $\mathrm{X}$ & $\mathrm{X}$ & $\mathrm{X}$ & $\mathrm{X}$ & $X$ & $\mathrm{X}$ & $\mathrm{X}$ & $\mathrm{X}$ & $\mathrm{X}$ \\
\hline 09032000 & Ranch Creek near Fraser, CO & Mountain & $\mathrm{X}$ & $\mathrm{X}$ & $\mathrm{X}$ & $\mathrm{X}$ & $\mathrm{X}$ & $\mathrm{X}$ & $\mathrm{X}$ & $\mathrm{X}$ & $\mathrm{X}$ & $\mathrm{X}$ \\
\hline 09032500 & Ranch Creek near Tabernash, CO & Mountain & $\mathrm{X}$ & $\mathrm{X}$ & $\mathrm{X}$ & $\mathrm{X}$ & $\mathrm{X}$ & $\mathrm{X}$ & -- & $\mathrm{X}$ & $\mathrm{X}$ & $\mathrm{X}$ \\
\hline 09033000 & Meadow Creek near Tabernash, CO & Mountain & $\mathrm{X}$ & $\mathrm{X}$ & $\mathrm{X}$ & $\mathrm{X}$ & $\mathrm{X}$ & $\mathrm{X}$ & $X$ & $\mathrm{X}$ & $\mathrm{X}$ & $\mathrm{X}$ \\
\hline 09033500 & Strawberry Creek near Granby, CO & Mountain & $\mathrm{X}$ & $\mathrm{X}$ & $\mathrm{X}$ & $\mathrm{X}$ & $\mathrm{X}$ & -- & -- & $\mathrm{X}$ & $\mathrm{X}$ & $\mathrm{X}$ \\
\hline 09034000 & Fraser River at Granby, $\mathrm{CO}$ & Mountain & $\mathrm{X}$ & $\mathrm{X}$ & $\mathrm{X}$ & $\mathrm{X}$ & $\mathrm{X}$ & $\mathrm{X}$ & $\mathrm{X}$ & $\mathrm{X}$ & $\mathrm{X}$ & $\mathrm{X}$ \\
\hline 09034500 & Colorado River at Hot Sulphur Springs, CO & Mountain & $\mathrm{X}$ & $\mathrm{X}$ & $\mathrm{X}$ & $\mathrm{X}$ & $\mathrm{X}$ & $\mathrm{X}$ & $\mathrm{X}$ & $\mathrm{X}$ & $\mathrm{X}$ & $\mathrm{X}$ \\
\hline 09034900 & Bobtail Creek near Jones Pass, CO & Mountain & $\mathrm{X}$ & $\mathrm{X}$ & $\mathrm{X}$ & $\mathrm{X}$ & $\mathrm{X}$ & $\mathrm{X}$ & $\mathrm{X}$ & $\mathrm{X}$ & $\mathrm{X}$ & $\mathrm{X}$ \\
\hline 09035500 & Williams Fork below Steelman Creek, CO & Mountain & $X$ & $\mathrm{X}$ & $X$ & $\mathrm{X}$ & $\mathrm{X}$ & $X$ & $\mathrm{X}$ & $\mathrm{X}$ & $\mathrm{X}$ & $\mathrm{X}$ \\
\hline 09035700 & Williams Fork above Darling Creek, Nr Leal, CO & Mountain & $\mathrm{X}$ & $\mathrm{X}$ & $\mathrm{X}$ & $\mathrm{X}$ & $\mathrm{X}$ & $\mathrm{X}$ & $\mathrm{X}$ & $\mathrm{X}$ & $\mathrm{X}$ & $\mathrm{X}$ \\
\hline 09035800 & Darling Creek near Leal, CO & Mountain & $\mathrm{X}$ & $\mathrm{X}$ & $\mathrm{X}$ & $\mathrm{X}$ & $\mathrm{X}$ & $\mathrm{X}$ & $\mathrm{X}$ & $\mathrm{X}$ & $\mathrm{X}$ & $\mathrm{X}$ \\
\hline 09035900 & South Fork of Williams Fork near Leal, CO & Mountain & $\mathrm{X}$ & $\mathrm{X}$ & $\mathrm{X}$ & $\mathrm{X}$ & $\mathrm{X}$ & $\mathrm{X}$ & $\mathrm{X}$ & $\mathrm{X}$ & $\mathrm{X}$ & $\mathrm{X}$ \\
\hline 09036500 & Keyser Creek near Leal, CO & Mountain & $\mathrm{X}$ & $\mathrm{X}$ & $\mathrm{X}$ & $\mathrm{X}$ & $\mathrm{X}$ & $\mathrm{X}$ & $\mathrm{X}$ & $\mathrm{X}$ & $\mathrm{X}$ & $\mathrm{X}$ \\
\hline 09039000 & Troublesome Creek near Pearmont, $\mathrm{CO}$ & Mountain & $\mathrm{X}$ & $\mathrm{X}$ & $\mathrm{X}$ & $\mathrm{X}$ & $\mathrm{X}$ & $\mathrm{X}$ & $\mathrm{X}$ & $\mathrm{X}$ & $\mathrm{X}$ & $\mathrm{X}$ \\
\hline 09040000 & East Fork Troublesome Creek near Troublesome, CO & Mountain & $\mathrm{X}$ & $\mathrm{X}$ & $\mathrm{X}$ & $\mathrm{X}$ & $\mathrm{X}$ & $\mathrm{X}$ & $\mathrm{X}$ & $\mathrm{X}$ & $\mathrm{X}$ & $\mathrm{X}$ \\
\hline 09041100 & Antelope Creek near Kremmling, $\mathrm{CO}$ & Mountain & $\mathrm{X}$ & $\mathrm{X}$ & $\mathrm{X}$ & $\mathrm{X}$ & $\mathrm{X}$ & $\mathrm{X}$ & -- & $\mathrm{X}$ & $\mathrm{X}$ & $\mathrm{X}$ \\
\hline 09046530 & French Gulch at Breckenridge, $\mathrm{CO}$ & Mountain & $\mathrm{X}$ & $\mathrm{X}$ & $\mathrm{X}$ & $\mathrm{X}$ & $\mathrm{X}$ & $\mathrm{X}$ & $\mathrm{X}$ & $\mathrm{X}$ & $\mathrm{X}$ & $\mathrm{X}$ \\
\hline 09047000 & Blue River at Dillon, CO & Mountain & $\mathrm{X}$ & $\mathrm{X}$ & $\mathrm{X}$ & $\mathrm{X}$ & $\mathrm{X}$ & $\mathrm{X}$ & $\mathrm{X}$ & $\mathrm{X}$ & $\mathrm{X}$ & $\mathrm{X}$ \\
\hline 09047500 & Snake River near Montezuma, CO & Mountain & $\mathrm{X}$ & $\mathrm{X}$ & $\mathrm{X}$ & $\mathrm{X}$ & $\mathrm{X}$ & $\mathrm{X}$ & $\mathrm{X}$ & $\mathrm{X}$ & $\mathrm{X}$ & $\mathrm{X}$ \\
\hline 09047700 & Keystone Gulch near Dillon, CO & Mountain & $\mathrm{X}$ & $\mathrm{X}$ & $\mathrm{X}$ & $\mathrm{X}$ & $\mathrm{X}$ & $\mathrm{X}$ & $\mathrm{X}$ & $\mathrm{X}$ & $\mathrm{X}$ & $\mathrm{X}$ \\
\hline 09050100 & Ten Mile Creek below North Ten Mile Creek, at Frisco, CO & Mountain & $\mathrm{X}$ & $\mathrm{X}$ & $\mathrm{X}$ & $\mathrm{X}$ & $\mathrm{X}$ & $\mathrm{X}$ & $\mathrm{X}$ & $\mathrm{X}$ & $\mathrm{X}$ & $\mathrm{X}$ \\
\hline 09052400 & Boulder Creek at Upper Station, near Dillon, CO & Mountain & $\mathrm{X}$ & $\mathrm{X}$ & $\mathrm{X}$ & $\mathrm{X}$ & $\mathrm{X}$ & $\mathrm{X}$ & $\mathrm{X}$ & $\mathrm{X}$ & $\mathrm{X}$ & $\mathrm{X}$ \\
\hline 09052800 & Slate Creek at Upper Station, near Dillon, CO & Mountain & $\mathrm{X}$ & $\mathrm{X}$ & $\mathrm{X}$ & $\mathrm{X}$ & $\mathrm{X}$ & $\mathrm{X}$ & $\mathrm{X}$ & $\mathrm{X}$ & $\mathrm{X}$ & $\mathrm{X}$ \\
\hline 09055000 & Otter Creek above Green Mountain Reservoir, CO & Mountain & $\mathrm{X}$ & $\mathrm{X}$ & $\mathrm{X}$ & $\mathrm{X}$ & $\mathrm{X}$ & $\mathrm{X}$ & $\mathrm{X}$ & $\mathrm{X}$ & $\mathrm{X}$ & $\mathrm{X}$ \\
\hline 09058610 & Dickson Creek near Vail, CO & Mountain & $\mathrm{X}$ & $\mathrm{X}$ & $\mathrm{X}$ & $\mathrm{X}$ & $\mathrm{X}$ & -- & -- & $\mathrm{X}$ & $\mathrm{X}$ & $\mathrm{X}$ \\
\hline 09058700 & Freeman Creek near Minturn, CO & Mountain & $\mathrm{X}$ & $\mathrm{X}$ & $\mathrm{X}$ & $\mathrm{X}$ & $\mathrm{X}$ & -- & -- & $\mathrm{X}$ & $\mathrm{X}$ & $\mathrm{X}$ \\
\hline 09058800 & East Meadow Creek near Minturn CO & Mountain & $\mathrm{X}$ & $\mathrm{X}$ & $\mathrm{X}$ & $\mathrm{X}$ & $\mathrm{X}$ & $\mathrm{X}$ & $\mathrm{X}$ & $\mathrm{X}$ & $\mathrm{X}$ & $\mathrm{X}$ \\
\hline 09059500 & Piney River near State Bridge, CO & Northwest & $\mathrm{X}$ & $\mathrm{X}$ & $\mathrm{X}$ & $\mathrm{X}$ & $\mathrm{X}$ & $\mathrm{X}$ & $\mathrm{X}$ & $\mathrm{X}$ & $\mathrm{X}$ & $\mathrm{X}$ \\
\hline 09060500 & Rock Creek near Toponas, CO & Mountain & $\mathrm{X}$ & $\mathrm{X}$ & $\mathrm{X}$ & $\mathrm{X}$ & $\mathrm{X}$ & $\mathrm{X}$ & $\mathrm{X}$ & $\mathrm{X}$ & $\mathrm{X}$ & $\mathrm{X}$ \\
\hline 09063200 & Wearyman Creek near Red Cliff, CO & Mountain & $\mathrm{X}$ & $\mathrm{X}$ & $\mathrm{X}$ & $\mathrm{X}$ & $\mathrm{X}$ & $\mathrm{X}$ & $\mathrm{X}$ & $\mathrm{X}$ & $\mathrm{X}$ & $\mathrm{X}$ \\
\hline 09063400 & Turkey Creek near Red Cliff, CO & Mountain & $\mathrm{X}$ & $\mathrm{X}$ & $\mathrm{X}$ & $\mathrm{X}$ & $\mathrm{X}$ & $\mathrm{X}$ & $\mathrm{X}$ & $\mathrm{X}$ & $\mathrm{X}$ & $\mathrm{X}$ \\
\hline 09064500 & Homestake Creek near Red Cliff, CO & Mountain & $\mathrm{X}$ & $\mathrm{X}$ & $\mathrm{X}$ & $\mathrm{X}$ & $\mathrm{X}$ & $\mathrm{X}$ & $\mathrm{X}$ & $\mathrm{X}$ & $\mathrm{X}$ & $\mathrm{X}$ \\
\hline
\end{tabular}


[Peak flow, 2-, 5-, 10-, 25-, 50-, 100-, 200-, and 500-year peak flow; Maximum ${ }_{7} Q_{2}$, maximum 7-day, 2-year flow; Maximum ${ }_{7} Q_{10}$, maximum 7-day, 10 -year flow; Maximum ${ }_{7} Q_{50}$, maximum 7-day, 50-year flow; Minimum ${ }_{7} Q_{2}$, minimum 7-day, 2-year flow; Minimum ${ }_{7} Q_{10}$, minimum 7-day, 10 -year flow; Minimum ${ }_{7} Q_{50}$, minimum 7-day, 50-year flow; Flow duration, 10-, 25-, 50-, 75-, and 90-percent flow duration; $\mathrm{X}$, station used in the development of regional regression equation for the indicated streamflow characteristic and hydrologic region; --, station not used in the development of regional regression equation for the indicated streamflow characteristic and hydrologic region]

\begin{tabular}{|c|c|c|c|c|c|c|c|c|c|c|c|c|}
\hline \multirow{3}{*}{$\begin{array}{l}\text { Station } \\
\text { number }\end{array}$} & \multirow{3}{*}{ Station name } & \multirow{3}{*}{$\begin{array}{l}\text { Hydrologic } \\
\text { region } \\
\text { (fig. 1) }\end{array}$} & \multicolumn{10}{|c|}{ Streamflow characteristic } \\
\hline & & & \multirow{2}{*}{$\begin{array}{l}\text { Peak } \\
\text { flow }\end{array}$} & \multicolumn{3}{|c|}{ Maximum } & \multicolumn{3}{|c|}{ Minimum } & \multicolumn{2}{|c|}{ Mean flow } & \multirow{2}{*}{$\begin{array}{c}\text { Flow } \\
\text { duration }\end{array}$} \\
\hline & & & & ${ }_{7} Q_{2}$ & ${ }_{7} Q_{10}$ & ${ }_{7} \boldsymbol{Q}_{50}$ & ${ }_{7} a_{2}$ & ${ }_{7} Q_{10}$ & ${ }_{7} \boldsymbol{Q}_{50}$ & Annual & Monthly & \\
\hline 09065100 & Cross Creek near Minturn, CO & Mountain & $\mathrm{X}$ & $\mathrm{X}$ & $\mathrm{X}$ & $\mathrm{X}$ & $\mathrm{X}$ & $\mathrm{X}$ & $\mathrm{X}$ & $\mathrm{X}$ & $\mathrm{X}$ & $\mathrm{X}$ \\
\hline 09065500 & Gore Creek at Upper Station, near Minturn, CO & Mountain & $\mathrm{X}$ & $\mathrm{X}$ & $\mathrm{X}$ & $\mathrm{X}$ & $\mathrm{X}$ & $\mathrm{X}$ & $\mathrm{X}$ & $\mathrm{X}$ & $\mathrm{X}$ & $\mathrm{X}$ \\
\hline 09066000 & Black Gore Creek near Minturn, CO & Mountain & $\mathrm{X}$ & $\mathrm{X}$ & $\mathrm{X}$ & $\mathrm{X}$ & $\mathrm{X}$ & $\mathrm{X}$ & $\mathrm{X}$ & $\mathrm{X}$ & $\mathrm{X}$ & $\mathrm{X}$ \\
\hline 09066100 & Bighorn Creek near Minturn, CO & Mountain & $\mathrm{X}$ & $\mathrm{X}$ & $\mathrm{X}$ & $\mathrm{X}$ & $\mathrm{X}$ & $X$ & $\mathrm{X}$ & $\mathrm{X}$ & $\mathrm{X}$ & $\mathrm{X}$ \\
\hline 09066150 & Pitkin Creek near Minturn, CO & Mountain & $\mathrm{X}$ & $\mathrm{X}$ & $\mathrm{X}$ & $\mathrm{X}$ & $\mathrm{X}$ & $\mathrm{X}$ & $\mathrm{X}$ & $\mathrm{X}$ & $\mathrm{X}$ & $\mathrm{X}$ \\
\hline 09066200 & Booth Creek near Minturn, CO & Mountain & $\mathrm{X}$ & $\mathrm{X}$ & $\mathrm{X}$ & $\mathrm{X}$ & $\mathrm{X}$ & $\mathrm{X}$ & $\mathrm{X}$ & $\mathrm{X}$ & $\mathrm{X}$ & $\mathrm{X}$ \\
\hline 09066300 & Middle Creek near Minturn, CO & Mountain & $\mathrm{X}$ & $\mathrm{X}$ & $\mathrm{X}$ & $\mathrm{X}$ & $\mathrm{X}$ & $\mathrm{X}$ & & $\mathrm{X}$ & $\mathrm{X}$ & $\mathrm{X}$ \\
\hline 09066400 & Red Sandstone Creek near Minturn, CO & Mountain & $\mathrm{X}$ & $\mathrm{X}$ & $\mathrm{X}$ & $\mathrm{X}$ & $\mathrm{X}$ & $\mathrm{X}$ & $\mathrm{X}$ & $\mathrm{X}$ & $\mathrm{X}$ & $\mathrm{X}$ \\
\hline 09066500 & Gore Creek near Minturn, CO & Mountain & $\mathrm{X}$ & $\mathrm{X}$ & $\mathrm{X}$ & $\mathrm{X}$ & $X$ & $\mathrm{X}$ & $\mathrm{X}$ & $\mathrm{X}$ & $\mathrm{X}$ & $\mathrm{X}$ \\
\hline 09067005 & Eagle River at Avon, $\mathrm{CO}$ & Mountain & $\mathrm{X}$ & $\mathrm{X}$ & $\mathrm{X}$ & $\mathrm{X}$ & $\mathrm{X}$ & $\mathrm{X}$ & $\mathrm{X}$ & $\mathrm{X}$ & $\mathrm{X}$ & $\mathrm{X}$ \\
\hline 09068000 & Brush Creek near Eagle, $\mathrm{CO}$ & Mountain & $\mathrm{X}$ & $\mathrm{X}$ & $\mathrm{X}$ & $\mathrm{X}$ & $\mathrm{X}$ & $\mathrm{X}$ & $\mathrm{X}$ & $\mathrm{X}$ & $\mathrm{X}$ & $\mathrm{X}$ \\
\hline 09069500 & Gypsum Creek near Gypsum, CO & Mountain & $\mathrm{X}$ & $\mathrm{X}$ & $\mathrm{X}$ & $\mathrm{X}$ & $\mathrm{X}$ & $\mathrm{X}$ & $\mathrm{X}$ & $\mathrm{X}$ & $\mathrm{X}$ & $\mathrm{X}$ \\
\hline 09070000 & Eagle River below Gypsum, CO & Mountain & $\mathrm{X}$ & $\mathrm{X}$ & $\mathrm{X}$ & $\mathrm{X}$ & $\mathrm{X}$ & $\mathrm{X}$ & $\mathrm{X}$ & $\mathrm{X}$ & $\mathrm{X}$ & $\mathrm{X}$ \\
\hline 09071300 & Grizzly Creek near Glenwood Springs, CO & Mountain & $\mathrm{X}$ & $\mathrm{X}$ & $\mathrm{X}$ & $\mathrm{X}$ & $\mathrm{X}$ & -- & -- & $\mathrm{X}$ & $\mathrm{X}$ & $\mathrm{X}$ \\
\hline 09073500 & Roaring Fork River at Aspen, $\mathrm{CO}$ & Mountain & $\mathrm{X}$ & $\mathrm{X}$ & $\mathrm{X}$ & $\mathrm{X}$ & $X$ & $\mathrm{X}$ & $\mathrm{X}$ & $\mathrm{X}$ & $\mathrm{X}$ & $\mathrm{X}$ \\
\hline 09073700 & Hunter Creek above Midway Creek, near Aspen, CO & Mountain & $\mathrm{X}$ & $\mathrm{X}$ & $\mathrm{X}$ & $\mathrm{X}$ & $\mathrm{X}$ & $\mathrm{X}$ & $\mathrm{X}$ & $\mathrm{X}$ & $\mathrm{X}$ & $\mathrm{X}$ \\
\hline 09073800 & Midway Creek near Aspen, $\mathrm{CO}$ & Mountain & $\mathrm{X}$ & $\mathrm{X}$ & $\mathrm{X}$ & $\mathrm{X}$ & $\mathrm{X}$ & $\mathrm{X}$ & $\mathrm{X}$ & $\mathrm{X}$ & $\mathrm{X}$ & $\mathrm{X}$ \\
\hline 09073900 & No Name Creek near Aspen, CO & Mountain & $\mathrm{X}$ & $\mathrm{X}$ & $\mathrm{X}$ & $\mathrm{X}$ & $\mathrm{X}$ & $\mathrm{X}$ & $\mathrm{X}$ & $\mathrm{X}$ & $\mathrm{X}$ & $\mathrm{X}$ \\
\hline 09074000 & Hunter Creek near Aspen, CO & Mountain & $\mathrm{X}$ & $\mathrm{X}$ & $\mathrm{X}$ & $\mathrm{X}$ & $\mathrm{X}$ & $\mathrm{X}$ & $\mathrm{X}$ & $\mathrm{X}$ & $\mathrm{X}$ & $\mathrm{X}$ \\
\hline 09074800 & Castle Creek Above Aspen, CO & Mountain & $\mathrm{X}$ & $\mathrm{X}$ & $\mathrm{X}$ & $\mathrm{X}$ & $\mathrm{X}$ & $\mathrm{X}$ & $\mathrm{X}$ & $\mathrm{X}$ & $\mathrm{X}$ & $\mathrm{X}$ \\
\hline 09075700 & Maroon Creek Above Aspen, CO & Mountain & $\mathrm{X}$ & $\mathrm{X}$ & $\mathrm{X}$ & $\mathrm{X}$ & $\mathrm{X}$ & $\mathrm{X}$ & $\mathrm{X}$ & $\mathrm{X}$ & $\mathrm{X}$ & $\mathrm{X}$ \\
\hline 09077200 & Fryingpan River near Ivanhoe Lake, CO & Mountain & $\mathrm{X}$ & $\mathrm{X}$ & $\mathrm{X}$ & $\mathrm{X}$ & $\mathrm{X}$ & $\mathrm{X}$ & $\mathrm{X}$ & $\mathrm{X}$ & $\mathrm{X}$ & $\mathrm{X}$ \\
\hline 09077800 & South Fork Fryingpan River at Upper Station, near Norrie, CO & Mountain & $\mathrm{X}$ & $\mathrm{X}$ & $\mathrm{X}$ & $\mathrm{X}$ & $\mathrm{X}$ & $\mathrm{X}$ & $\mathrm{X}$ & $\mathrm{X}$ & $\mathrm{X}$ & $\mathrm{X}$ \\
\hline 09078000 & Fryingpan River at Norrie, CO & Mountain & $\mathrm{X}$ & $\mathrm{X}$ & $\mathrm{X}$ & $\mathrm{X}$ & $\mathrm{X}$ & $\mathrm{X}$ & $\mathrm{X}$ & $\mathrm{X}$ & $\mathrm{X}$ & $\mathrm{X}$ \\
\hline 09078100 & $\begin{array}{l}\text { North Fork Fryingpan River above Cunningham C, near } \\
\text { Norrie, CO }\end{array}$ & Mountain & $\mathrm{X}$ & $\mathrm{X}$ & $\mathrm{X}$ & $\mathrm{X}$ & $\mathrm{X}$ & $\mathrm{X}$ & $\mathrm{X}$ & $\mathrm{X}$ & $\mathrm{X}$ & $\mathrm{X}$ \\
\hline 09078200 & Cunningham Creek near Norrie, $\mathrm{CO}$ & Mountain & $\mathrm{X}$ & $\mathrm{X}$ & $\mathrm{X}$ & $\mathrm{X}$ & $\mathrm{X}$ & $\mathrm{X}$ & $\mathrm{X}$ & $\mathrm{X}$ & $\mathrm{X}$ & $\mathrm{X}$ \\
\hline 09078500 & North Fork Fryingpan River near Norrie, CO & Mountain & $\mathrm{X}$ & $\mathrm{X}$ & $\mathrm{X}$ & $\mathrm{X}$ & $\mathrm{X}$ & $\mathrm{X}$ & $\mathrm{X}$ & $\mathrm{X}$ & $\mathrm{X}$ & $\mathrm{X}$ \\
\hline 09080300 & Rocky Fork Creek near Meredith, CO & Mountain & $\mathrm{X}$ & $\mathrm{X}$ & $\mathrm{X}$ & $\mathrm{X}$ & $\mathrm{X}$ & $\mathrm{X}$ & $\mathrm{X}$ & $X$ & $\mathrm{X}$ & $\mathrm{X}$ \\
\hline 09081600 & Crystal River above Avalanche Creek, near Redstone, CO & Northwest & $\mathrm{X}$ & $\mathrm{X}$ & $\mathrm{X}$ & $\mathrm{X}$ & $\mathrm{X}$ & $\mathrm{X}$ & $\mathrm{X}$ & $\mathrm{X}$ & $\mathrm{X}$ & $\mathrm{X}$ \\
\hline 09082800 & North Thompson Creek near Carbondale, $\mathrm{CO}$ & Mountain & $\mathrm{X}$ & $\mathrm{X}$ & $\mathrm{X}$ & $\mathrm{X}$ & $\mathrm{X}$ & $\mathrm{X}$ & $\mathrm{X}$ & $\mathrm{X}$ & $\mathrm{X}$ & $\mathrm{X}$ \\
\hline 09083000 & Thompson Creek near Carbondale, $\mathrm{CO}$ & Northwest & $\mathrm{X}$ & $\mathrm{X}$ & $\mathrm{X}$ & $\mathrm{X}$ & $\mathrm{X}$ & $\mathrm{X}$ & $\mathrm{X}$ & $\mathrm{X}$ & $\mathrm{X}$ & $\mathrm{X}$ \\
\hline 09084000 & Cattle Creek near Carbondale, $\mathrm{CO}$ & Mountain & $\mathrm{X}$ & $\mathrm{X}$ & $\mathrm{X}$ & $\mathrm{X}$ & $\mathrm{X}$ & $\mathrm{X}$ & $\mathrm{X}$ & $\mathrm{X}$ & $\mathrm{X}$ & $\mathrm{X}$ \\
\hline 09085200 & Canyon Creek above New Castle, CO & Northwest & $\mathrm{X}$ & $\mathrm{X}$ & $\mathrm{X}$ & $\mathrm{X}$ & $\mathrm{X}$ & $\mathrm{X}$ & $\mathrm{X}$ & $X$ & $\mathrm{X}$ & $\mathrm{X}$ \\
\hline 09085300 & East Canyon Creek near New Castle, CO & Northwest & $\mathrm{X}$ & $\mathrm{X}$ & $\mathrm{X}$ & $\mathrm{X}$ & $\mathrm{X}$ & $\mathrm{X}$ & $\mathrm{X}$ & $\mathrm{X}$ & $\mathrm{X}$ & $\mathrm{X}$ \\
\hline 09085400 & Possum Creek near New Castle, CO & Northwest & $\mathrm{X}$ & $\mathrm{X}$ & $\mathrm{X}$ & $\mathrm{X}$ & $\mathrm{X}$ & $\mathrm{X}$ & $\mathrm{X}$ & $\mathrm{X}$ & $\mathrm{X}$ & $\mathrm{X}$ \\
\hline 09089500 & West Divide Creek near Raven, CO & Mountain & $\mathrm{X}$ & $\mathrm{X}$ & $\mathrm{X}$ & $\mathrm{X}$ & $\mathrm{X}$ & -- & -- & $\mathrm{X}$ & $\mathrm{X}$ & $\mathrm{X}$ \\
\hline 09091100 & Mamm Creek near Silt, CO & Northwest & $\mathrm{X}$ & -- & -- & -- & -- & -- & -- & -- & -- & -- \\
\hline 09091500 & East Rifle Creek near Rifle, CO & Northwest & $\mathrm{X}$ & $\mathrm{X}$ & $\mathrm{X}$ & $\mathrm{X}$ & $\mathrm{X}$ & $\mathrm{X}$ & $\mathrm{X}$ & $\mathrm{X}$ & $\mathrm{X}$ & $\mathrm{X}$ \\
\hline
\end{tabular}


Table 4. Streamflow-gaging stations used in the development of the regional streamflow regression equations.-Continued

[Peak flow, 2-, 5-, 10-, 25-, 50-, 100-, 200-, and 500-year peak flow; Maximum ${ }_{7} Q_{2}$, maximum 7-day, 2-year flow; Maximum ${ }_{7} Q_{10}$, maximum 7-day, 10 -year flow; Maximum ${ }_{7} Q_{50}$, maximum 7-day, 50-year flow; Minimum ${ }_{7} Q_{2}$, minimum 7-day, 2-year flow; Minimum ${ }_{7} Q_{10}$, minimum 7-day, 10-year flow; Minimum ${ }_{7} Q_{50}$, minimum 7-day, 50-year flow; Flow duration, 10-, 25-, 50-, 75-, and 90-percent flow duration; $\mathrm{X}$, station used in the development of regional regression equation for the indicated streamflow characteristic and hydrologic region; --, station not used in the development of regional regression equation for the indicated streamflow characteristic and hydrologic region]

\begin{tabular}{|c|c|c|c|c|c|c|c|c|c|c|c|c|}
\hline \multirow{3}{*}{$\begin{array}{l}\text { Station } \\
\text { number }\end{array}$} & \multirow{3}{*}{ Station name } & \multirow{3}{*}{$\begin{array}{l}\text { Hydrologic } \\
\text { region } \\
\text { (fig. 1) }\end{array}$} & \multicolumn{10}{|c|}{ Streamflow characteristic } \\
\hline & & & \multirow{2}{*}{$\begin{array}{l}\text { Peak } \\
\text { flow }\end{array}$} & \multicolumn{3}{|c|}{ Maximum } & \multicolumn{3}{|c|}{ Minimum } & \multicolumn{2}{|c|}{ Mean flow } & \multirow{2}{*}{$\begin{array}{c}\text { Flow } \\
\text { duration }\end{array}$} \\
\hline & & & & ${ }_{7} Q_{2}$ & ${ }_{7} Q_{10}$ & ${ }_{7} a_{50}$ & ${ }_{7} a_{2}$ & ${ }_{7} Q_{10}$ & ${ }_{7} Q_{50}$ & Annual & Monthly & \\
\hline 09092000 & Rifle Creek near Rifle, CO & Northwest & $\mathrm{X}$ & $\mathrm{X}$ & $\mathrm{X}$ & $\mathrm{X}$ & $\mathrm{X}$ & $\mathrm{X}$ & $\mathrm{X}$ & $\mathrm{X}$ & $\mathrm{X}$ & $\mathrm{X}$ \\
\hline 09092500 & Beaver Creek near Rifle, $\mathrm{CO}$ & Northwest & $\mathrm{X}$ & $\mathrm{X}$ & $\mathrm{X}$ & $\mathrm{X}$ & $\mathrm{X}$ & $X$ & $\mathrm{X}$ & $\mathrm{X}$ & $\mathrm{X}$ & $\mathrm{X}$ \\
\hline 09093000 & Parachute Creek near Parachute CO & Northwest & $\mathrm{X}$ & $\mathrm{X}$ & $\mathrm{X}$ & $\mathrm{X}$ & $\mathrm{X}$ & -- & - & $\mathrm{X}$ & $\mathrm{X}$ & $\mathrm{X}$ \\
\hline 09095000 & Roan Creek near De Beque, CO & Northwest & $\mathrm{X}$ & $\mathrm{X}$ & $\mathrm{X}$ & $\mathrm{X}$ & $\mathrm{X}$ & $\mathrm{X}$ & $\mathrm{X}$ & $\mathrm{X}$ & $\mathrm{X}$ & $\mathrm{X}$ \\
\hline 09096000 & Plateau Creek at Upper Station, near Collbran, CO & Mountain & $\mathrm{X}$ & $\mathrm{X}$ & $\mathrm{X}$ & $\mathrm{X}$ & $\mathrm{X}$ & $\mathrm{X}$ & -- & $\mathrm{X}$ & $\mathrm{X}$ & $\mathrm{X}$ \\
\hline 09096500 & Plateau Creek near Collbran, $\mathrm{CO}$ & Mountain & $\mathrm{X}$ & $\mathrm{X}$ & $\mathrm{X}$ & $\mathrm{X}$ & $\mathrm{X}$ & $\mathrm{X}$ & $\mathrm{X}$ & $\mathrm{X}$ & $\mathrm{X}$ & $\mathrm{X}$ \\
\hline 09096800 & Buzzard Creek below Owens Creek, near Heiberger, CO & Mountain & $\mathrm{X}$ & $\mathrm{X}$ & $\mathrm{X}$ & $\mathrm{X}$ & -- & -- & -- & $\mathrm{X}$ & $\mathrm{X}$ & $\mathrm{X}$ \\
\hline 09097500 & Buzzard Creek near Collbran, CO & Mountain & $\mathrm{X}$ & $\mathrm{X}$ & $\mathrm{X}$ & $\mathrm{X}$ & $\mathrm{X}$ & $\mathrm{X}$ & -- & $\mathrm{X}$ & $\mathrm{X}$ & $\mathrm{X}$ \\
\hline 09097600 & Brush Creek near Collbran, CO & Mountain & $\mathrm{X}$ & $\mathrm{X}$ & $\mathrm{X}$ & $\mathrm{X}$ & $\mathrm{X}$ & $\mathrm{X}$ & $\mathrm{X}$ & $\mathrm{X}$ & $\mathrm{X}$ & $\mathrm{X}$ \\
\hline 09104500 & Mesa Creek near Mesa, CO & Northwest & $\mathrm{X}$ & $\mathrm{X}$ & $\mathrm{X}$ & $X$ & $\mathrm{X}$ & $X$ & $\mathrm{X}$ & $X$ & $\mathrm{X}$ & $\mathrm{X}$ \\
\hline 09107000 & Taylor River at Taylor Park, CO & Mountain & $\mathrm{X}$ & $\mathrm{X}$ & $\mathrm{X}$ & $X$ & $X$ & $X$ & $\mathrm{X}$ & $\mathrm{X}$ & $X$ & $\mathrm{X}$ \\
\hline 09110000 & Taylor River at Almont, CO & Mountain & $\mathrm{X}$ & $\mathrm{X}$ & $\mathrm{X}$ & $X$ & $\mathrm{X}$ & $X$ & $\mathrm{X}$ & $\mathrm{X}$ & $\mathrm{X}$ & $\mathrm{X}$ \\
\hline 09110500 & East River near Crested Butte, CO & Mountain & $\mathrm{X}$ & $\mathrm{X}$ & $\mathrm{X}$ & $X$ & $\mathrm{X}$ & $X$ & $\mathrm{X}$ & $\mathrm{X}$ & $X$ & $\mathrm{X}$ \\
\hline 09111500 & Slate River near Crested Butte, CO & Mountain & $\mathrm{X}$ & $\mathrm{X}$ & $\mathrm{X}$ & $X$ & $X$ & $X$ & $\mathrm{X}$ & $\mathrm{X}$ & $X$ & $\mathrm{X}$ \\
\hline 09112000 & Cement Creek near Crested Butte, CO & Mountain & $X$ & $\mathrm{X}$ & $\mathrm{X}$ & $\mathrm{X}$ & $\mathrm{X}$ & $\mathrm{X}$ & $\mathrm{X}$ & $\mathrm{X}$ & $\mathrm{X}$ & $\mathrm{X}$ \\
\hline 09112500 & East River at Almont CO & Mountain & $\mathrm{X}$ & $\mathrm{X}$ & $\mathrm{X}$ & $\mathrm{X}$ & $\mathrm{X}$ & $\mathrm{X}$ & $\mathrm{X}$ & $\mathrm{X}$ & $\mathrm{X}$ & $\mathrm{X}$ \\
\hline 09113300 & Ohio Creek at Baldwin, $\mathrm{CO}$ & Mountain & $\mathrm{X}$ & $\mathrm{X}$ & $\mathrm{X}$ & $\mathrm{X}$ & $\mathrm{X}$ & $\mathrm{X}$ & $\mathrm{X}$ & $\mathrm{X}$ & $\mathrm{X}$ & $\mathrm{X}$ \\
\hline 09113500 & Ohio Creek near Baldwin, CO & Mountain & $\mathrm{X}$ & $\mathrm{X}$ & $\mathrm{X}$ & $\mathrm{X}$ & $\mathrm{X}$ & $\mathrm{X}$ & $\mathrm{X}$ & $\mathrm{X}$ & $\mathrm{X}$ & $\mathrm{X}$ \\
\hline 09114500 & Gunnison River near Gunnison, CO & Mountain & $\mathrm{X}$ & $\mathrm{X}$ & $\mathrm{X}$ & $\mathrm{X}$ & $\mathrm{X}$ & $\mathrm{X}$ & $\mathrm{X}$ & $\mathrm{X}$ & $\mathrm{X}$ & $\mathrm{X}$ \\
\hline 09115500 & Tomichi Creek at Sargents, CO & Mountain & $\mathrm{X}$ & $\mathrm{X}$ & $\mathrm{X}$ & $\mathrm{X}$ & $\mathrm{X}$ & $\mathrm{X}$ & $\mathrm{X}$ & $\mathrm{X}$ & $\mathrm{X}$ & $\mathrm{X}$ \\
\hline 09117000 & Tomichi Creek at Parlin, CO & Mountain & $\mathrm{X}$ & $\mathrm{X}$ & $\mathrm{X}$ & $\mathrm{X}$ & $\mathrm{X}$ & $\mathrm{X}$ & $\mathrm{X}$ & $\mathrm{X}$ & $\mathrm{X}$ & $\mathrm{X}$ \\
\hline 09118000 & Quartz Creek near Ohio City, CO & Mountain & $\mathrm{X}$ & $\mathrm{X}$ & $\mathrm{X}$ & $\mathrm{X}$ & $\mathrm{X}$ & $\mathrm{X}$ & $\mathrm{X}$ & $\mathrm{X}$ & $\mathrm{X}$ & $\mathrm{X}$ \\
\hline 09119000 & Tomichi Creek at Gunnison, CO & Mountain & $\mathrm{X}$ & $\mathrm{X}$ & $\mathrm{X}$ & $\mathrm{X}$ & $\mathrm{X}$ & $\mathrm{X}$ & $\mathrm{X}$ & $\mathrm{X}$ & $X$ & $\mathrm{X}$ \\
\hline 09122000 & Cebolla Creek at Powderhorn, $\mathrm{CO}$ & Mountain & $\mathrm{X}$ & $\mathrm{X}$ & $\mathrm{X}$ & $X$ & $\mathrm{X}$ & $\mathrm{X}$ & $\mathrm{X}$ & $\mathrm{X}$ & $\mathrm{X}$ & $\mathrm{X}$ \\
\hline 09122500 & Soap Creek near Sapinero, CO & Mountain & $\mathrm{X}$ & $\mathrm{X}$ & $X$ & $X$ & $X$ & $X$ & $\mathrm{X}$ & $X$ & $X$ & $\mathrm{X}$ \\
\hline 09123500 & Lake Fork at Lake City, CO & Mountain & $X$ & $X$ & $\mathrm{X}$ & $\mathrm{X}$ & $\mathrm{X}$ & $X$ & $X$ & $X$ & $\mathrm{X}$ & $\mathrm{X}$ \\
\hline 09124500 & Lake Fork at Gateview, CO & Mountain & $\mathrm{X}$ & $\mathrm{X}$ & $\mathrm{X}$ & $\mathrm{X}$ & $\mathrm{X}$ & $\mathrm{X}$ & $\mathrm{X}$ & $\mathrm{X}$ & $\mathrm{X}$ & $\mathrm{X}$ \\
\hline 09125000 & Curecanti Creek near Sapinero, CO & Mountain & $\mathrm{X}$ & $\mathrm{X}$ & $\mathrm{X}$ & $\mathrm{X}$ & $\mathrm{X}$ & $\mathrm{X}$ & $\mathrm{X}$ & $\mathrm{X}$ & $\mathrm{X}$ & $\mathrm{X}$ \\
\hline 09126000 & Cimarron River near Cimarron, $\mathrm{CO}$ & Mountain & $\mathrm{X}$ & $\mathrm{X}$ & $\mathrm{X}$ & $\mathrm{X}$ & $\mathrm{X}$ & $\mathrm{X}$ & $\mathrm{X}$ & $\mathrm{X}$ & $\mathrm{X}$ & $\mathrm{X}$ \\
\hline 09127500 & Crystal Creek near Maher, CO & Mountain & $\mathrm{X}$ & $\mathrm{X}$ & $\mathrm{X}$ & $\mathrm{X}$ & $\mathrm{X}$ & $\mathrm{X}$ & $\mathrm{X}$ & $\mathrm{X}$ & $\mathrm{X}$ & $\mathrm{X}$ \\
\hline 09128500 & Smith Fork near Crawford, CO & Northwest & $\mathrm{X}$ & $\mathrm{X}$ & $\mathrm{X}$ & $\mathrm{X}$ & $\mathrm{X}$ & $\mathrm{X}$ & $\mathrm{X}$ & $\mathrm{X}$ & $\mathrm{X}$ & $\mathrm{X}$ \\
\hline 09130500 & East Muddy Creek near Bardine, $\mathrm{CO}$ & Northwest & $\mathrm{X}$ & $\mathrm{X}$ & $\mathrm{X}$ & $X$ & $\mathrm{X}$ & $\mathrm{X}$ & $\mathrm{X}$ & $\mathrm{X}$ & $\mathrm{X}$ & $\mathrm{X}$ \\
\hline 09130600 & West Muddy Creek near Ragged Mountain, CO & Mountain & $\mathrm{X}$ & $\mathrm{X}$ & $\mathrm{X}$ & $\mathrm{X}$ & $\mathrm{X}$ & $X$ & $\mathrm{X}$ & $\mathrm{X}$ & $\mathrm{X}$ & $\mathrm{X}$ \\
\hline 09132500 & North Fork Gunnison River near Somerset, CO & Northwest & $\mathrm{X}$ & $\mathrm{X}$ & $\mathrm{X}$ & $\mathrm{X}$ & $\mathrm{X}$ & $\mathrm{X}$ & $\mathrm{X}$ & $\mathrm{X}$ & $\mathrm{X}$ & $\mathrm{X}$ \\
\hline 09132900 & West Hubbard Creek near Paonia, CO & Mountain & $\mathrm{X}$ & $\mathrm{X}$ & $\mathrm{X}$ & $\mathrm{X}$ & $\mathrm{X}$ & $\mathrm{X}$ & $\mathrm{X}$ & $\mathrm{X}$ & $\mathrm{X}$ & $\mathrm{X}$ \\
\hline 09134500 & Leroux Creek near Cedaredge, $\mathrm{CO}$ & Northwest & $\mathrm{X}$ & $\mathrm{X}$ & $\mathrm{X}$ & $\mathrm{X}$ & $\mathrm{X}$ & $\mathrm{X}$ & $\mathrm{X}$ & $\mathrm{X}$ & $\mathrm{X}$ & $\mathrm{X}$ \\
\hline 09136200 & Gunnison River near Lazear, CO & Northwest & $\mathrm{X}$ & $\mathrm{X}$ & $\mathrm{X}$ & $X$ & $\mathrm{X}$ & $X$ & $\mathrm{X}$ & $\mathrm{X}$ & $\mathrm{X}$ & $\mathrm{X}$ \\
\hline 09137800 & Dirty George Creek near Grand Mesa, CO & Northwest & $\mathrm{X}$ & $\mathrm{X}$ & $\mathrm{X}$ & $\mathrm{X}$ & $\mathrm{X}$ & $\mathrm{X}$ & $\mathrm{X}$ & $\mathrm{X}$ & $\mathrm{X}$ & $\mathrm{X}$ \\
\hline 09139200 & Ward Creek near Grand Mesa, CO & Mountain & $\mathrm{X}$ & $\mathrm{X}$ & $\mathrm{X}$ & $\mathrm{X}$ & $\mathrm{X}$ & $\mathrm{X}$ & $\mathrm{X}$ & $\mathrm{X}$ & $\mathrm{X}$ & $\mathrm{X}$ \\
\hline
\end{tabular}


[Peak flow, 2-, 5-, 10-, 25-, 50-, 100-, 200-, and 500-year peak flow; Maximum ${ }_{7} Q_{2}$, maximum 7-day, 2-year flow; Maximum ${ }_{7} Q_{10}$, maximum 7-day, 10 -year flow; Maximum ${ }_{7} Q_{50}$, maximum 7-day, 50-year flow; Minimum ${ }_{7} Q_{2}$, minimum 7-day, 2-year flow; Minimum ${ }_{7} Q_{10}$, minimum 7-day, 10-year flow; Minimum ${ }_{7} Q_{50}$, minimum 7-day, 50-year flow; Flow duration, 10-, 25-, 50-, 75-, and 90-percent flow duration; $\mathrm{X}$, station used in the development of regional regression equation for the indicated streamflow characteristic and hydrologic region; --, station not used in the development of regional regression equation for the indicated streamflow characteristic and hydrologic region]

\begin{tabular}{|c|c|c|c|c|c|c|c|c|c|c|c|c|}
\hline \multirow{3}{*}{$\begin{array}{l}\text { Station } \\
\text { number }\end{array}$} & \multirow{3}{*}{ Station name } & \multirow{3}{*}{$\begin{array}{l}\text { Hydrologic } \\
\text { region } \\
\text { (fig. 1) }\end{array}$} & \multicolumn{10}{|c|}{ Streamflow characteristic } \\
\hline & & & \multirow{2}{*}{$\begin{array}{l}\text { Peak } \\
\text { flow }\end{array}$} & \multicolumn{3}{|c|}{ Maximum } & \multicolumn{3}{|c|}{ Minimum } & \multicolumn{2}{|c|}{ Mean flow } & \multirow{2}{*}{$\begin{array}{c}\text { Flow } \\
\text { duration }\end{array}$} \\
\hline & & & & ${ }_{7} a_{2}$ & ${ }_{7} Q_{10}$ & ${ }_{7} Q_{50}$ & ${ }_{7} a_{2}$ & ${ }_{7} Q_{10}$ & ${ }_{7} Q_{50}$ & Annual & Monthly & \\
\hline 09140200 & Kiser Creek near Grand Mesa, CO & Mountain & $\mathrm{X}$ & $\mathrm{X}$ & $\mathrm{X}$ & $\mathrm{X}$ & $\mathrm{X}$ & $\mathrm{X}$ & $\mathrm{X}$ & $\mathrm{X}$ & $\mathrm{X}$ & $\mathrm{X}$ \\
\hline 09141200 & Youngs Creek near Grand Mesa, CO & Northwest & $\mathrm{X}$ & $\mathrm{X}$ & $\mathrm{X}$ & $\mathrm{X}$ & $X$ & $X$ & $\mathrm{X}$ & $\mathrm{X}$ & $\mathrm{X}$ & $\mathrm{X}$ \\
\hline 09143500 & Surface Creek at Cedaredge, CO & Northwest & $\mathrm{X}$ & $\mathrm{X}$ & $\mathrm{X}$ & $\mathrm{X}$ & $\mathrm{X}$ & $\mathrm{X}$ & $\mathrm{X}$ & $\mathrm{X}$ & $\mathrm{X}$ & $\mathrm{X}$ \\
\hline 09144200 & Tongue Creek at Cory, CO & Northwest & $\mathrm{X}$ & $\mathrm{X}$ & $\mathrm{X}$ & $\mathrm{X}$ & $\mathrm{X}$ & $\mathrm{X}$ & $\mathrm{X}$ & $\mathrm{X}$ & $\mathrm{X}$ & $\mathrm{X}$ \\
\hline 09146000 & Uncompahgre River below Ouray, $\mathrm{CO}$ & Southwest & $\mathrm{X}$ & $\mathrm{X}$ & $X$ & $\mathrm{X}$ & $\mathrm{X}$ & $\mathrm{X}$ & $\mathrm{X}$ & $\mathrm{X}$ & $\mathrm{X}$ & $\mathrm{X}$ \\
\hline 09146400 & West Fork Dallas Creek near Ridgway, CO & Southwest & $\mathrm{X}$ & $\mathrm{X}$ & $\mathrm{X}$ & $\mathrm{X}$ & $\mathrm{X}$ & $\mathrm{X}$ & $\mathrm{X}$ & $\mathrm{X}$ & $\mathrm{X}$ & $\mathrm{X}$ \\
\hline 09146500 & East Fork Dallas Creek near Ridgway, CO & Southwest & $\mathrm{X}$ & $\mathrm{X}$ & $\mathrm{X}$ & $\mathrm{X}$ & $\mathrm{X}$ & $\mathrm{X}$ & $\mathrm{X}$ & $\mathrm{X}$ & $\mathrm{X}$ & $\mathrm{X}$ \\
\hline 09146600 & Pleasant Valley Creek near Noel, CO & Southwest & $\mathrm{X}$ & $\mathrm{X}$ & $\mathrm{X}$ & $\mathrm{X}$ & $X$ & $\mathrm{X}$ & & $X$ & $X$ & $X$ \\
\hline 09147000 & Dallas Creek near Ridgway, CO & Southwest & $\mathrm{X}$ & $\mathrm{X}$ & $\mathrm{X}$ & $\mathrm{X}$ & $\mathrm{X}$ & $\mathrm{X}$ & $X$ & $\mathrm{X}$ & $\mathrm{X}$ & $\mathrm{X}$ \\
\hline 09147100 & Cow Creek near Ridgway, CO & Mountain & $\mathrm{X}$ & $\mathrm{X}$ & $X$ & $\mathrm{X}$ & $\mathrm{X}$ & $\mathrm{X}$ & $\mathrm{X}$ & $X$ & $\mathrm{X}$ & $\mathrm{X}$ \\
\hline 09149450 & Dry Creek near Olathe, CO & Northwest & $\mathrm{X}$ & -- & -- & -- & -- & -- & -- & -- & -- & -- \\
\hline 09150500 & Roubideau Creek at Mouth, near Delta, CO & Northwest & $\mathrm{X}$ & $\mathrm{X}$ & $\mathrm{X}$ & $\mathrm{X}$ & $\mathrm{X}$ & $\mathrm{X}$ & $\mathrm{X}$ & $\mathrm{X}$ & $\mathrm{X}$ & $\mathrm{X}$ \\
\hline 09153400 & West Salt Creek near Mack, CO & Northwest & $\mathrm{X}$ & $\mathrm{X}$ & $\mathrm{X}$ & $\mathrm{X}$ & -- & -- & -- & $\mathrm{X}$ & $\mathrm{X}$ & $\mathrm{X}$ \\
\hline 09163700 & Cisco Wash near Cisco, UT & Southwest & $\mathrm{X}$ & & & & -- & -- & -- & & & \\
\hline 09165000 & Dolores River below Rico, CO & Southwest & $\mathrm{X}$ & $\mathrm{X}$ & $\mathrm{X}$ & $\mathrm{X}$ & -- & -- & -- & $\mathrm{X}$ & $\mathrm{X}$ & $\mathrm{X}$ \\
\hline 09166500 & Dolores River at Dolores, $\mathrm{CO}$ & Southwest & $\mathrm{X}$ & $\mathrm{X}$ & $\mathrm{X}$ & $\mathrm{X}$ & $\mathrm{X}$ & $\mathrm{X}$ & $\mathrm{X}$ & $\mathrm{X}$ & $\mathrm{X}$ & $\mathrm{X}$ \\
\hline 09168100 & Disappointment Creek near Dove Creek, CO & Southwest & $\mathrm{X}$ & $\mathrm{X}$ & $\mathrm{X}$ & $\mathrm{X}$ & $\mathrm{X}$ & -- & -- & $\mathrm{X}$ & $\mathrm{X}$ & $\mathrm{X}$ \\
\hline 09168700 & Disappointment Creek Tributary near Slick Rock, CO & Southwest & $\mathrm{X}$ & -- & -- & -- & -- & -- & -- & -- & -- & -- \\
\hline 09169500 & Dolores River at Bedrock, $\mathrm{CO}$ & Southwest & $\mathrm{X}$ & $\mathrm{X}$ & $\mathrm{X}$ & $\mathrm{X}$ & $\mathrm{X}$ & $\mathrm{X}$ & $\mathrm{X}$ & $\mathrm{X}$ & $\mathrm{X}$ & $\mathrm{X}$ \\
\hline 09172000 & Fall Creek near Fall Creek, CO & Southwest & $\mathrm{X}$ & $\mathrm{X}$ & $\mathrm{X}$ & $\mathrm{X}$ & $X$ & $X$ & $\mathrm{X}$ & $\mathrm{X}$ & $\mathrm{X}$ & $\mathrm{X}$ \\
\hline 09172500 & San Miguel River near Placerville, CO & Southwest & $\mathrm{X}$ & $\mathrm{X}$ & $\mathrm{X}$ & $\mathrm{X}$ & $\mathrm{X}$ & $\mathrm{X}$ & $\mathrm{X}$ & $\mathrm{X}$ & $\mathrm{X}$ & $\mathrm{X}$ \\
\hline 09174500 & Cottonwood Creek near Nucla, CO & Southwest & $\mathrm{X}$ & $\mathrm{X}$ & $\mathrm{X}$ & $\mathrm{X}$ & & -- & -- & $\mathrm{X}$ & $\mathrm{X}$ & $\mathrm{X}$ \\
\hline 09175000 & West Naturita Creek near Norwood, CO & Southwest & $\mathrm{X}$ & $\mathrm{X}$ & $X$ & $\mathrm{X}$ & $X$ & -- & -- & $\mathrm{X}$ & $\mathrm{X}$ & $\mathrm{X}$ \\
\hline 09175500 & San Miguel River at Naturita, CO & Southwest & $\mathrm{X}$ & $\mathrm{X}$ & $\mathrm{X}$ & $\mathrm{X}$ & $\mathrm{X}$ & $\mathrm{X}$ & $\mathrm{X}$ & $\mathrm{X}$ & $\mathrm{X}$ & $\mathrm{X}$ \\
\hline 09177500 & Taylor Creek near Gateway, CO & Southwest & $\mathrm{X}$ & -- & -- & -- & -- & -- & -- & -- & -- & -- \\
\hline 09181000 & Onion Creek near Moab, UT & Southwest & $\mathrm{X}$ & $\mathrm{X}$ & $\mathrm{X}$ & $\mathrm{X}$ & $\mathrm{X}$ & $\mathrm{X}$ & $\mathrm{X}$ & $\mathrm{X}$ & $\mathrm{X}$ & $\mathrm{X}$ \\
\hline 09182000 & Castle Creek above Diversions, near Moab, UT & Southwest & $\mathrm{X}$ & $\mathrm{X}$ & $\mathrm{X}$ & $\mathrm{X}$ & $\mathrm{X}$ & -- & -- & $\mathrm{X}$ & $\mathrm{X}$ & $\mathrm{X}$ \\
\hline 09182600 & Salt Wash near Thompson, UT & Southwest & $\mathrm{X}$ & -- & -- & -- & - & -- & -- & - & - & - \\
\hline 09183000 & Courthouse Wash near Moab, UT & Southwest & $\mathrm{X}$ & $\mathrm{X}$ & $\mathrm{X}$ & $\mathrm{X}$ & $\mathrm{X}$ & -- & -- & $\mathrm{X}$ & $\mathrm{X}$ & $\mathrm{X}$ \\
\hline 09184000 & Mill Creek near Moab, UT & Southwest & $\mathrm{X}$ & $\mathrm{X}$ & $\mathrm{X}$ & $\mathrm{X}$ & $\mathrm{X}$ & $\mathrm{X}$ & $\mathrm{X}$ & $\mathrm{X}$ & $\mathrm{X}$ & $\mathrm{X}$ \\
\hline 09185200 & Kane Springs Canyon near Moab, UT & Southwest & $\mathrm{X}$ & -- & -- & -- & -- & -- & -- & -- & -- & -- \\
\hline 09185500 & Hatch Wash near La Sal, UT & Southwest & $\mathrm{X}$ & $\mathrm{X}$ & $\mathrm{X}$ & $\mathrm{X}$ & -- & -- & -- & $\mathrm{X}$ & $\mathrm{X}$ & $\mathrm{X}$ \\
\hline 09187000 & Cottonwood Creek near Monticello, UT & Southwest & $\mathrm{X}$ & $\mathrm{X}$ & $\mathrm{X}$ & $\mathrm{X}$ & -- & -- & -- & $\mathrm{X}$ & $\mathrm{X}$ & $\mathrm{X}$ \\
\hline 09216400 & Greasewood Canyon near Green River, WY & Northwest & $\mathrm{X}$ & -- & -- & -- & -- & -- & -- & -- & -- & -- \\
\hline 09216537 & Delaney Draw near Red Desert, WY & Northwest & $\mathrm{X}$ & -- & -- & -- & -- & -- & -- & -- & -- & -- \\
\hline 09216550 & Deadman Wash near Point of Rocks, WY & Northwest & $\mathrm{X}$ & -- & -- & -- & -- & -- & -- & -- & -- & -- \\
\hline 09216600 & Cutthroat Draw near Rock Springs, WY & Northwest & $\mathrm{X}$ & -- & -- & -- & -- & -- & -- & -- & -- & -- \\
\hline 09216700 & Salt Wells Creek near Rock Springs, WY & Northwest & $\mathrm{X}$ & -- & -- & -- & -- & -- & -- & -- & -- & -- \\
\hline 09216900 & Bitter Cr Tributary near Green River WY & Northwest & $\mathrm{X}$ & -- & -- & -- & -- & -- & -- & -- & -- & -- \\
\hline
\end{tabular}


Table 4. Streamflow-gaging stations used in the development of the regional streamflow regression equations.-Continued

[Peak flow, 2-, 5-, 10-, 25-, 50-, 100-, 200-, and 500-year peak flow; Maximum ${ }_{7} Q_{2}$, maximum 7-day, 2-year flow; Maximum ${ }_{7} Q_{10}$, maximum 7-day, 10 -year flow; Maximum ${ }_{7} Q_{50}$, maximum 7-day, 50-year flow; Minimum ${ }_{7} Q_{2}$, minimum 7-day, 2-year flow; Minimum ${ }_{7} Q_{10}$, minimum 7-day, 10-year flow; Minimum ${ }_{7} Q_{50}$, minimum 7-day, 50-year flow; Flow duration, 10-, 25-, 50-, 75-, and 90-percent flow duration; $\mathrm{X}$, station used in the development of regional regression equation for the indicated streamflow characteristic and hydrologic region; --, station not used in the development of regional regression equation for the indicated streamflow characteristic and hydrologic region]

\begin{tabular}{|c|c|c|c|c|c|c|c|c|c|c|c|c|}
\hline \multirow{3}{*}{$\begin{array}{l}\text { Station } \\
\text { number }\end{array}$} & \multirow{3}{*}{ Station name } & \multirow{3}{*}{$\begin{array}{l}\text { Hydrologic } \\
\text { region } \\
\text { (fig. 1) }\end{array}$} & \multicolumn{10}{|c|}{ Streamflow characteristic } \\
\hline & & & \multirow{2}{*}{$\begin{array}{l}\text { Peak } \\
\text { flow }\end{array}$} & \multicolumn{3}{|c|}{ Maximum } & \multicolumn{3}{|c|}{ Minimum } & \multicolumn{2}{|c|}{ Mean flow } & \multirow{2}{*}{$\begin{array}{c}\text { Flow } \\
\text { duration }\end{array}$} \\
\hline & & & & ${ }_{7} Q_{2}$ & ${ }_{7} Q_{10}$ & ${ }_{7} Q_{50}$ & ${ }_{7} \boldsymbol{Q}_{2}$ & ${ }_{7} Q_{10}$ & ${ }_{7} Q_{50}$ & Annual & Monthly & \\
\hline 09224810 & Blacks Fork Tributary No 2 near Green River, WY & Northwest & $\mathrm{X}$ & -- & -- & -- & -- & -- & -- & -- & -- & -- \\
\hline 09224820 & Blacks Fork Tributary No 3 near Green River, WY & Northwest & $\mathrm{X}$ & -- & -- & -- & -- & -- & -- & -- & -- & -- \\
\hline 09224840 & Blacks Fork Tributary No 4 near Green River, WY & Northwest & $\mathrm{X}$ & -- & -- & -- & -- & -- & -- & -- & -- & -- \\
\hline 09224980 & Summers Dry Creek near Green River, WY & Northwest & $\mathrm{X}$ & -- & -- & -- & -- & -- & -- & -- & -- & -- \\
\hline 09225200 & Squaw Hollow near Burntfork, WY & Northwest & $\mathrm{X}$ & -- & -- & -- & -- & -- & -- & -- & -- & -- \\
\hline 09225300 & Green River Tributary No 2 near Burntfork, WY & Northwest & $\mathrm{X}$ & -- & -- & -- & -- & -- & -- & -- & -- & -- \\
\hline 09229450 & Henrys Fork Tributary near Manila, UT & Northwest & $\mathrm{X}$ & -- & -- & -- & -- & -- & -- & -- & -- & -- \\
\hline 09235600 & Pot Creek Above Diversions, near Vernal, UT & Northwest & $\mathrm{X}$ & $\mathrm{X}$ & $\mathrm{X}$ & $\mathrm{X}$ & -- & -- & -- & $\mathrm{X}$ & $\mathrm{X}$ & $\mathrm{X}$ \\
\hline 09238500 & Walton Creek near Steamboat Springs, CO & Northwest & $X$ & $\mathrm{X}$ & $\mathrm{X}$ & $\mathrm{X}$ & $\mathrm{X}$ & $\mathrm{X}$ & $\mathrm{X}$ & $\mathrm{X}$ & $\mathrm{X}$ & $\mathrm{X}$ \\
\hline 09239500 & Yampa River at Steamboat Springs, CO & Northwest & $\mathrm{X}$ & $\mathrm{X}$ & $\mathrm{X}$ & $\mathrm{X}$ & $\mathrm{X}$ & $\mathrm{X}$ & $\mathrm{X}$ & $\mathrm{X}$ & $\mathrm{X}$ & $\mathrm{X}$ \\
\hline 09241000 & Elk River at Clark, CO & Mountain & $\mathrm{X}$ & $\mathrm{X}$ & $\mathrm{X}$ & $\mathrm{X}$ & $\mathrm{X}$ & $\mathrm{X}$ & $\mathrm{X}$ & $\mathrm{X}$ & $\mathrm{X}$ & $\mathrm{X}$ \\
\hline 09244100 & Fish Creek near Milner, CO & Northwest & $\mathrm{X}$ & $\mathrm{X}$ & $\mathrm{X}$ & $\mathrm{X}$ & $\mathrm{X}$ & $\mathrm{X}$ & $\mathrm{X}$ & $\mathrm{X}$ & $\mathrm{X}$ & $\mathrm{X}$ \\
\hline 09245000 & Elkhead Creek near Elkhead, CO & Northwest & $\mathrm{X}$ & $\mathrm{X}$ & $\mathrm{X}$ & $\mathrm{X}$ & $\mathrm{X}$ & $\mathrm{X}$ & -- & $\mathrm{X}$ & $\mathrm{X}$ & $\mathrm{X}$ \\
\hline 09245500 & North Fork Elkhead Creek near Elkhead, CO & Northwest & $\mathrm{X}$ & $\mathrm{X}$ & $\mathrm{X}$ & $\mathrm{X}$ & $\mathrm{X}$ & -- & -- & $\mathrm{X}$ & $\mathrm{X}$ & $\mathrm{X}$ \\
\hline 09249000 & East Fork Williams Fork near Pagoda, CO & Northwest & $\mathrm{X}$ & $\mathrm{X}$ & $\mathrm{X}$ & $\mathrm{X}$ & $\mathrm{X}$ & $\mathrm{X}$ & $\mathrm{X}$ & $\mathrm{X}$ & $\mathrm{X}$ & $\mathrm{X}$ \\
\hline 09250000 & Milk Creek near Thornburgh, CO & Northwest & $X$ & -- & -- & -- & -- & -- & -- & -- & -- & -- \\
\hline 09251500 & Middle Fork Little Snake River near Battle Creek, CO & Northwest & $\mathrm{X}$ & $\mathrm{X}$ & $\mathrm{X}$ & $\mathrm{X}$ & $\mathrm{X}$ & $\mathrm{X}$ & $\mathrm{X}$ & $\mathrm{X}$ & $\mathrm{X}$ & $\mathrm{X}$ \\
\hline 09251800 & North Fork Little Snake River near Encampment, WY & Northwest & $\mathrm{X}$ & $\mathrm{X}$ & $\mathrm{X}$ & $\mathrm{X}$ & $\mathrm{X}$ & $\mathrm{X}$ & $\mathrm{X}$ & $\mathrm{X}$ & $\mathrm{X}$ & $\mathrm{X}$ \\
\hline 09253000 & Little Snake River near Slater, CO & Northwest & $\mathrm{X}$ & $\mathrm{X}$ & $\mathrm{X}$ & $\mathrm{X}$ & $\mathrm{X}$ & $\mathrm{X}$ & $\mathrm{X}$ & $\mathrm{X}$ & $\mathrm{X}$ & $\mathrm{X}$ \\
\hline 09253400 & Battle Creek near Encampment, WY & Northwest & $\mathrm{X}$ & $\mathrm{X}$ & $\mathrm{X}$ & $\mathrm{X}$ & $X$ & $\mathrm{X}$ & $X$ & -- & -- & -- \\
\hline 09255000 & Slater Fork near Slater, CO & Northwest & $\mathrm{X}$ & $\mathrm{X}$ & $\mathrm{X}$ & $\mathrm{X}$ & $\mathrm{X}$ & $\mathrm{X}$ & $\mathrm{X}$ & $\mathrm{X}$ & $\mathrm{X}$ & $\mathrm{X}$ \\
\hline 09256000 & Savery Creek near Savery, WY & Northwest & $\mathrm{X}$ & $\mathrm{X}$ & $\mathrm{X}$ & $\mathrm{X}$ & $\mathrm{X}$ & $\mathrm{X}$ & -- & $\mathrm{X}$ & $\mathrm{X}$ & $\mathrm{X}$ \\
\hline 09257000 & Little Snake River near Dixon, WY & Northwest & $\mathrm{X}$ & $\mathrm{X}$ & $\mathrm{X}$ & $\mathrm{X}$ & $\mathrm{X}$ & $\mathrm{X}$ & $\mathrm{X}$ & $\mathrm{X}$ & $\mathrm{X}$ & $\mathrm{X}$ \\
\hline 09257500 & Willow Creek near Baggs, WY & Northwest & $\mathrm{X}$ & -- & -- & -- & -- & -- & -- & -- & -- & -- \\
\hline 09258000 & Willow Creek near Dixon, WY & Northwest & $\mathrm{X}$ & $\mathrm{X}$ & $\mathrm{X}$ & $\mathrm{X}$ & $\mathrm{X}$ & $\mathrm{X}$ & -- & $\mathrm{X}$ & $\mathrm{X}$ & $\mathrm{X}$ \\
\hline 09258200 & Dry Cow Creek near Baggs, WY & Northwest & $\mathrm{X}$ & -- & -- & -- & -- & -- & -- & -- & -- & -- \\
\hline 09258900 & Muddy Creek above Baggs, WY & Northwest & $\mathrm{X}$ & -- & -- & -- & -- & -- & -- & -- & -- & -- \\
\hline 09259500 & Fourmile Creek near Baggs, WY & Northwest & $\mathrm{X}$ & -- & -- & -- & -- & -- & -- & -- & -- & -- \\
\hline 09260000 & Little Snake River near Lily, CO & Northwest & $\mathrm{X}$ & $\mathrm{X}$ & $\mathrm{X}$ & $\mathrm{X}$ & $\mathrm{X}$ & -- & -- & $\mathrm{X}$ & $\mathrm{X}$ & $\mathrm{X}$ \\
\hline 09263700 & Cliff Creek near Jensen, UT & Northwest & $\mathrm{X}$ & -- & -- & -- & -- & -- & -- & -- & -- & -- \\
\hline 09263800 & Cow Wash near Jensen, UT & Northwest & $\mathrm{X}$ & -- & -- & -- & -- & -- & -- & -- & -- & -- \\
\hline 09264000 & Ashley C Below Trout C near Vernal, UT & Northwest & $\mathrm{X}$ & $\mathrm{X}$ & $\mathrm{X}$ & $\mathrm{X}$ & $\mathrm{X}$ & $\mathrm{X}$ & $\mathrm{X}$ & $\mathrm{X}$ & $\mathrm{X}$ & $\mathrm{X}$ \\
\hline 09264500 & South Fork Ashley Creek nearVernal, UT & Northwest & $\mathrm{X}$ & $\mathrm{X}$ & $\mathrm{X}$ & $\mathrm{X}$ & $\mathrm{X}$ & $\mathrm{X}$ & $\mathrm{X}$ & $\mathrm{X}$ & $\mathrm{X}$ & $\mathrm{X}$ \\
\hline 09266500 & Ashley Creek near Vernal, UT & Northwest & $\mathrm{X}$ & $\mathrm{X}$ & $\mathrm{X}$ & $\mathrm{X}$ & $\mathrm{X}$ & $\mathrm{X}$ & $\mathrm{X}$ & $\mathrm{X}$ & $\mathrm{X}$ & $\mathrm{X}$ \\
\hline 09268000 & Dry Fork Above Sinks, near Dry Fork, UT & Northwest & $\mathrm{X}$ & $\mathrm{X}$ & $\mathrm{X}$ & $\mathrm{X}$ & $\mathrm{X}$ & $\mathrm{X}$ & $\mathrm{X}$ & $\mathrm{X}$ & $\mathrm{X}$ & $\mathrm{X}$ \\
\hline 09268500 & North Fork Of Dry Fork near Dry Fork, UT & Northwest & $\mathrm{X}$ & $\mathrm{X}$ & $\mathrm{X}$ & $\mathrm{X}$ & $\mathrm{X}$ & $\mathrm{X}$ & $\mathrm{X}$ & $\mathrm{X}$ & $\mathrm{X}$ & $\mathrm{X}$ \\
\hline 09268900 & Brownie Canyon Above Sinks, near Dry Fork, UT & Northwest & $\mathrm{X}$ & $\mathrm{X}$ & $\mathrm{X}$ & $\mathrm{X}$ & $\mathrm{X}$ & $\mathrm{X}$ & $\mathrm{X}$ & $\mathrm{X}$ & $\mathrm{X}$ & $\mathrm{X}$ \\
\hline 09269000 & East Fork Of Dry Fork near Dry Fork, UT & Northwest & $\mathrm{X}$ & $\mathrm{X}$ & $\mathrm{X}$ & $\mathrm{X}$ & -- & -- & -- & $\mathrm{X}$ & $\mathrm{X}$ & $\mathrm{X}$ \\
\hline 09270500 & Dry Fork at Mouth near Dry Fork, UT & Northwest & $\mathrm{X}$ & $\mathrm{X}$ & $\mathrm{X}$ & $\mathrm{X}$ & $\mathrm{X}$ & -- & -- & $\mathrm{X}$ & $\mathrm{X}$ & $\mathrm{X}$ \\
\hline
\end{tabular}


[Peak flow, 2-, 5-, 10-, 25-, 50-, 100-, 200-, and 500-year peak flow; Maximum ${ }_{7} Q_{2}$, maximum 7-day, 2-year flow; Maximum ${ }_{7} Q_{10}$, maximum 7-day, 10 -year flow; Maximum ${ }_{7} Q_{50}$, maximum 7-day, 50-year flow; Minimum ${ }_{7} Q_{2}$, minimum 7-day, 2-year flow; Minimum ${ }_{7} Q_{10}$, minimum 7-day, 10 -year flow; Minimum ${ }_{7} Q_{50}$, minimum 7-day, 50-year flow; Flow duration, 10-, 25-, 50-, 75-, and 90-percent flow duration; $\mathrm{X}$, station used in the development of regional regression equation for the indicated streamflow characteristic and hydrologic region; --, station not used in the development of regional regression equation for the indicated streamflow characteristic and hydrologic region]

\begin{tabular}{|c|c|c|c|c|c|c|c|c|c|c|c|c|}
\hline \multirow{3}{*}{$\begin{array}{l}\text { Station } \\
\text { number }\end{array}$} & \multirow{3}{*}{ Station name } & \multirow{3}{*}{$\begin{array}{l}\text { Hydrologic } \\
\text { region } \\
\text { (fig. 1) }\end{array}$} & \multicolumn{10}{|c|}{ Streamflow characteristic } \\
\hline & & & \multirow{2}{*}{$\begin{array}{l}\text { Peak } \\
\text { flow }\end{array}$} & \multicolumn{3}{|c|}{ Maximum } & \multicolumn{3}{|c|}{ Minimum } & \multicolumn{2}{|c|}{ Mean flow } & \multirow{2}{*}{$\begin{array}{c}\text { Flow } \\
\text { duration }\end{array}$} \\
\hline & & & & ${ }_{7} Q_{2}$ & ${ }_{7} Q_{10}$ & ${ }_{7} Q_{50}$ & ${ }_{7} a_{2}$ & ${ }_{7} Q_{10}$ & ${ }_{7} \boldsymbol{O}_{50}$ & Annual & Monthly & \\
\hline 09271800 & Halfway Hollow Tributary near Lapoint, UT & Northwest & $\mathrm{X}$ & -- & -- & -- & -- & -- & -- & -- & -- & -- \\
\hline 09300500 & Uintah River at Fort Duchesne, UT & Northwest & $\mathrm{X}$ & $\mathrm{X}$ & $\mathrm{X}$ & $\mathrm{X}$ & $\mathrm{X}$ & $\mathrm{X}$ & -- & $\mathrm{X}$ & $\mathrm{X}$ & $\mathrm{X}$ \\
\hline 09302450 & Lost Creek near Buford, CO & Mountain & $\mathrm{X}$ & $\mathrm{X}$ & $\mathrm{X}$ & $\mathrm{X}$ & $X$ & $\mathrm{X}$ & $\mathrm{X}$ & $\mathrm{X}$ & $\mathrm{X}$ & $\mathrm{X}$ \\
\hline 09302500 & Marvine Creek near Buford, CO & Mountain & $\mathrm{X}$ & $X$ & $\mathrm{X}$ & $\mathrm{X}$ & $X$ & $\mathrm{X}$ & $\mathrm{X}$ & $\mathrm{X}$ & $X$ & $\mathrm{X}$ \\
\hline 09303000 & North Fork White River at Buford, CO & Northwest & $\mathrm{X}$ & $\mathrm{X}$ & $\mathrm{X}$ & $\mathrm{X}$ & $\mathrm{X}$ & $\mathrm{X}$ & $\mathrm{X}$ & $\mathrm{X}$ & $\mathrm{X}$ & $\mathrm{X}$ \\
\hline 09303300 & South Fork White River at Budges Resort, CO & Mountain & $\mathrm{X}$ & $\mathrm{X}$ & $\mathrm{X}$ & $\mathrm{X}$ & $\mathrm{X}$ & $\mathrm{X}$ & $\mathrm{X}$ & $\mathrm{X}$ & $\mathrm{X}$ & $\mathrm{X}$ \\
\hline 09303320 & Wagonwheel Creek at Budges Resort, CO & Mountain & $\mathrm{X}$ & $\mathrm{X}$ & $\mathrm{X}$ & $\mathrm{X}$ & -- & -- & -- & $\mathrm{X}$ & $\mathrm{X}$ & $\mathrm{X}$ \\
\hline 09304000 & South Fork White River at Buford, CO & Northwest & $\mathrm{X}$ & $\mathrm{X}$ & $\mathrm{X}$ & $\mathrm{X}$ & -- & -- & -- & $\mathrm{X}$ & $\mathrm{X}$ & $\mathrm{X}$ \\
\hline 09304500 & White River near Meeker, CO & Northwest & $\mathrm{X}$ & $\mathrm{X}$ & $\mathrm{X}$ & $\mathrm{X}$ & $\mathrm{X}$ & $\mathrm{X}$ & $\mathrm{X}$ & $\mathrm{X}$ & $\mathrm{X}$ & $\mathrm{X}$ \\
\hline 09306007 & Piceance Creek below Rio Blanco, CO & Northwest & $\mathrm{X}$ & $\mathrm{X}$ & $\mathrm{X}$ & $\mathrm{X}$ & $\mathrm{X}$ & $\mathrm{X}$ & $\mathrm{X}$ & $\mathrm{X}$ & $\mathrm{X}$ & $\mathrm{X}$ \\
\hline 09306036 & Sorghum Gulch at mouth, near Rio Blanco, $\mathrm{CO}$ & Northwest & $\mathrm{X}$ & -- & -- & -- & -- & -- & -- & -- & -- & $\mathrm{X}$ \\
\hline 09306039 & Cottonwood Gulch near Rio Blanco, CO & Northwest & $\mathrm{X}$ & -- & -- & -- & -- & -- & -- & -- & -- & $\mathrm{X}$ \\
\hline 09306042 & Piceance Creek Tributary near Rio Blanco, CO & Northwest & $\mathrm{X}$ & -- & -- & -- & -- & -- & -- & -- & $\mathrm{X}$ & $\mathrm{X}$ \\
\hline 09306052 & Scandard Gulch at Mouth, near Rio Blanco, CO & Northwest & $\mathrm{X}$ & -- & -- & -- & -- & -- & -- & -- & $\mathrm{X}$ & $\mathrm{X}$ \\
\hline 09306058 & Willow Creek near Rio Blanco, $\mathrm{CO}$ & Northwest & $\mathrm{X}$ & $\mathrm{X}$ & $\mathrm{X}$ & $\mathrm{X}$ & -- & -- & -- & $\mathrm{X}$ & $\mathrm{X}$ & $\mathrm{X}$ \\
\hline 09306200 & Piceance Creek below Ryan Gulch, near Rio Blanco, CO & Northwest & $\mathrm{X}$ & $\mathrm{X}$ & $\mathrm{X}$ & $\mathrm{X}$ & $\mathrm{X}$ & $\mathrm{X}$ & $\mathrm{X}$ & $\mathrm{X}$ & $\mathrm{X}$ & $\mathrm{X}$ \\
\hline 09306240 & Box Elder Gulch near Rangely, CO & Northwest & $\mathrm{X}$ & $\mathrm{X}$ & $\mathrm{X}$ & $\mathrm{X}$ & -- & -- & -- & $\mathrm{X}$ & & $\mathrm{X}$ \\
\hline 09306242 & Corral Gulch near Rangely, CO & Northwest & $\mathrm{X}$ & $\mathrm{X}$ & $\mathrm{X}$ & $\mathrm{X}$ & -- & -- & -- & $\mathrm{X}$ & $\mathrm{X}$ & $\mathrm{X}$ \\
\hline 09306255 & Yellow Creek near White River, CO & Northwest & $\mathrm{X}$ & $\mathrm{X}$ & $\mathrm{X}$ & $\mathrm{X}$ & $\mathrm{X}$ & $\mathrm{X}$ & -- & $\mathrm{X}$ & $\mathrm{X}$ & $\mathrm{X}$ \\
\hline 09306800 & Bitter Creek near Bonanza, UT & Northwest & $\mathrm{X}$ & $\mathrm{X}$ & $\mathrm{X}$ & $\mathrm{X}$ & -- & -- & -- & $\mathrm{X}$ & $\mathrm{X}$ & $\mathrm{X}$ \\
\hline 09307500 & Willow Creek above Diversions near Ouray, UT & Northwest & $\mathrm{X}$ & $\mathrm{X}$ & $\mathrm{X}$ & $\mathrm{X}$ & $\mathrm{X}$ & $\mathrm{X}$ & $\mathrm{X}$ & $\mathrm{X}$ & $\mathrm{X}$ & $\mathrm{X}$ \\
\hline 09308000 & Willow Creek near Ouray, UT & Northwest & $\mathrm{X}$ & $\mathrm{X}$ & $\mathrm{X}$ & $\mathrm{X}$ & $\mathrm{X}$ & -- & -- & $\mathrm{X}$ & $X$ & $\mathrm{X}$ \\
\hline 09328900 & Cresent Wash near Cresent Junction, UT & Southwest & $\mathrm{X}$ & -- & -- & -- & -- & -- & -- & -- & -- & -- \\
\hline 09339900 & $\begin{array}{l}\text { East Fork San Juan River above Sand Creek, } \\
\text { near Pagosa Springs, CO }\end{array}$ & Southwest & $\mathrm{X}$ & $\mathrm{X}$ & $\mathrm{X}$ & $\mathrm{X}$ & $\mathrm{X}$ & $\mathrm{X}$ & $\mathrm{X}$ & $\mathrm{X}$ & $\mathrm{X}$ & $\mathrm{X}$ \\
\hline 09340000 & East Fork San Juan River near Pagosa Springs, CO & Southwest & $\mathrm{X}$ & $\mathrm{X}$ & $\mathrm{X}$ & $\mathrm{X}$ & $\mathrm{X}$ & $\mathrm{X}$ & $\mathrm{X}$ & $\mathrm{X}$ & $\mathrm{X}$ & $\mathrm{X}$ \\
\hline 09340500 & $\begin{array}{l}\text { West Fork San Juan River above Borns Lake, } \\
\text { near Pagosa Springs, CO }\end{array}$ & Southwest & $\mathrm{X}$ & $\mathrm{X}$ & $\mathrm{X}$ & $\mathrm{X}$ & $\mathrm{X}$ & $\mathrm{X}$ & $\mathrm{X}$ & $\mathrm{X}$ & $\mathrm{X}$ & $\mathrm{X}$ \\
\hline 09341500 & West Fork San Juan River near Pagosa Springs, CO & Southwest & $\mathrm{X}$ & $\mathrm{X}$ & $\mathrm{X}$ & $\mathrm{X}$ & $\mathrm{X}$ & $\mathrm{X}$ & $\mathrm{X}$ & $\mathrm{X}$ & $\mathrm{X}$ & $\mathrm{X}$ \\
\hline 09342000 & Turkey Creek near Pagosa Springs, CO & Southwest & $\mathrm{X}$ & $\mathrm{X}$ & $\mathrm{X}$ & $\mathrm{X}$ & $\mathrm{X}$ & $\mathrm{X}$ & $\mathrm{X}$ & $\mathrm{X}$ & $\mathrm{X}$ & $\mathrm{X}$ \\
\hline 09342500 & San Juan River at Pagosa Springs, CO & Southwest & $\mathrm{X}$ & $\mathrm{X}$ & $\mathrm{X}$ & $\mathrm{X}$ & $\mathrm{X}$ & $\mathrm{X}$ & $\mathrm{X}$ & $\mathrm{X}$ & $\mathrm{X}$ & $\mathrm{X}$ \\
\hline 09343000 & Rio Blanco near Pagosa Springs, CO & Southwest & $\mathrm{X}$ & $\mathrm{X}$ & $\mathrm{X}$ & $\mathrm{X}$ & $\mathrm{X}$ & $\mathrm{X}$ & $\mathrm{X}$ & $\mathrm{X}$ & $\mathrm{X}$ & $\mathrm{X}$ \\
\hline 09343500 & Rito Blanco near Pagosa Springs, CO & Southwest & $\mathrm{X}$ & $\mathrm{X}$ & $\mathrm{X}$ & $\mathrm{X}$ & $\mathrm{X}$ & -- & -- & $\mathrm{X}$ & $\mathrm{X}$ & $\mathrm{X}$ \\
\hline 09344000 & Navajo River at Banded Peak Ranch, near Chromo, CO & Southwest & $\mathrm{X}$ & $\mathrm{X}$ & $\mathrm{X}$ & $\mathrm{X}$ & $\mathrm{X}$ & $\mathrm{X}$ & $\mathrm{X}$ & $\mathrm{X}$ & $\mathrm{X}$ & $\mathrm{X}$ \\
\hline 09345200 & $\begin{array}{l}\text { Little Navajo River Below Lake Oso Diversion Dam, } \\
\text { near Chromo, CO }\end{array}$ & Southwest & $\mathrm{X}$ & $\mathrm{X}$ & $\mathrm{X}$ & $\mathrm{X}$ & $\mathrm{X}$ & $\mathrm{X}$ & -- & $\mathrm{X}$ & $\mathrm{X}$ & $\mathrm{X}$ \\
\hline 09346000 & Navajo River at Edith, $\mathrm{CO}$ & Southwest & $\mathrm{X}$ & $\mathrm{X}$ & $\mathrm{X}$ & $\mathrm{X}$ & $\mathrm{X}$ & $\mathrm{X}$ & $\mathrm{X}$ & $\mathrm{X}$ & $\mathrm{X}$ & $\mathrm{X}$ \\
\hline 09346200 & Rio Amargo at Dulce, NM & Southwest & $\mathrm{X}$ & -- & -- & -- & -- & -- & -- & -- & -- & -- \\
\hline 09347500 & Piedra $\mathrm{R}$ at Bridge Ranger Station, near Pagosa Spgs, CO & Southwest & $\mathrm{X}$ & $\mathrm{X}$ & $\mathrm{X}$ & $\mathrm{X}$ & $\mathrm{X}$ & $\mathrm{X}$ & $\mathrm{X}$ & $\mathrm{X}$ & $X$ & $X$ \\
\hline
\end{tabular}


Table 4. Streamflow-gaging stations used in the development of the regional streamflow regression equations.-Continued

[Peak flow, 2-, 5-, 10-, 25-, 50-, 100-, 200-, and 500-year peak flow; Maximum ${ }_{7} Q_{2}$, maximum 7-day, 2-year flow; Maximum ${ }_{7} Q_{10}$, maximum 7-day, 10 -year flow; Maximum ${ }_{7} Q_{50}$, maximum 7-day, 50-year flow; Minimum ${ }_{7} Q_{2}$, minimum 7-day, 2-year flow; Minimum ${ }_{7} Q_{10}$, minimum 7-day, 10-year flow; Minimum ${ }_{7} Q_{50}$, minimum 7-day, 50-year flow; Flow duration, 10-, 25-, 50-, 75-, and 90-percent flow duration; $\mathrm{X}$, station used in the development of regional regression equation for the indicated streamflow characteristic and hydrologic region; --, station not used in the development of regional regression equation for the indicated streamflow characteristic and hydrologic region]

\begin{tabular}{|c|c|c|c|c|c|c|c|c|c|c|c|c|}
\hline \multirow{3}{*}{$\begin{array}{l}\text { Station } \\
\text { number }\end{array}$} & \multirow{3}{*}{ Station name } & \multirow{3}{*}{$\begin{array}{l}\text { Hydrologic } \\
\text { region } \\
\text { (fig. 1) }\end{array}$} & \multicolumn{10}{|c|}{ Streamflow characteristic } \\
\hline & & & \multirow{2}{*}{$\begin{array}{l}\text { Peak } \\
\text { flow }\end{array}$} & \multicolumn{3}{|c|}{ Maximum } & \multicolumn{3}{|c|}{ Minimum } & \multicolumn{2}{|c|}{ Mean flow } & \multirow{2}{*}{$\begin{array}{c}\text { Flow } \\
\text { duration }\end{array}$} \\
\hline & & & & ${ }_{7} \mathrm{O}_{2}$ & ${ }_{7} Q_{10}$ & ${ }_{7} \boldsymbol{O}_{50}$ & ${ }_{7} O_{2}$ & ${ }_{7} Q_{10}$ & ${ }_{7} \boldsymbol{Q}_{50}$ & Annual & Monthly & \\
\hline 09349800 & Piedra River near Arboles, CO & Southwest & $\mathrm{X}$ & $\mathrm{X}$ & $\mathrm{X}$ & $\mathrm{X}$ & $\mathrm{X}$ & $\mathrm{X}$ & $\mathrm{X}$ & $\mathrm{X}$ & $\mathrm{X}$ & $\mathrm{X}$ \\
\hline 09350800 & Vaqueros Canyon near Gobernador, NM & Southwest & $\mathrm{X}$ & -- & -- & -- & -- & -- & -- & -- & -- & -- \\
\hline 09352900 & Vallecito Creek near Bayfield, CO & Southwest & $\mathrm{X}$ & $\mathrm{X}$ & $\mathrm{X}$ & $\mathrm{X}$ & $\mathrm{X}$ & $\mathrm{X}$ & $\mathrm{X}$ & $X$ & $\mathrm{X}$ & $\mathrm{X}$ \\
\hline 09355000 & Spring Creek at La Boca, CO & Southwest & $\mathrm{X}$ & $\mathrm{X}$ & $\mathrm{X}$ & $\mathrm{X}$ & $\mathrm{X}$ & $\mathrm{X}$ & $\mathrm{X}$ & $\mathrm{X}$ & $\mathrm{X}$ & $\mathrm{X}$ \\
\hline 09357200 & Gallegos Canyon Tributary near Nageezi, NM & Southwest & $\mathrm{X}$ & -- & -- & -- & -- & -- & -- & -- & -- & -- \\
\hline 09357230 & West Draw near Farmington, NM & Southwest & $\mathrm{X}$ & -- & -- & -- & -- & -- & -- & -- & -- & -- \\
\hline 09357500 & Animas River at Howardsville, $\mathrm{CO}$ & Southwest & $\mathrm{X}$ & $\mathrm{X}$ & $\mathrm{X}$ & $\mathrm{X}$ & -- & -- & -- & $X$ & $\mathrm{X}$ & $\mathrm{X}$ \\
\hline 09358550 & Cement Creek at Silverton, $\mathrm{CO}$ & Southwest & $\mathrm{X}$ & $\mathrm{X}$ & $X$ & $\mathrm{X}$ & $X$ & $\mathrm{X}$ & $X$ & $X$ & $\mathrm{X}$ & $\mathrm{X}$ \\
\hline 09359000 & Mineral Creek near Silverton, CO & Southwest & $\mathrm{X}$ & $\mathrm{X}$ & $X$ & $\mathrm{X}$ & $X$ & $\mathrm{X}$ & $X$ & $X$ & $\mathrm{X}$ & $\mathrm{X}$ \\
\hline 09361000 & Hermosa Creek near Hermosa, CO & Southwest & $\mathrm{X}$ & $\mathrm{X}$ & $\mathrm{X}$ & $\mathrm{X}$ & $\mathrm{X}$ & $X$ & $\mathrm{X}$ & $\mathrm{X}$ & $\mathrm{X}$ & $\mathrm{X}$ \\
\hline 09361400 & Junction Creek near Durango, CO & Southwest & $X$ & $\mathrm{X}$ & $\mathrm{X}$ & $\mathrm{X}$ & $\mathrm{X}$ & $\mathrm{X}$ & $X$ & $\mathrm{X}$ & $\mathrm{X}$ & $\mathrm{X}$ \\
\hline 09361500 & Animas River at Durango, $\mathrm{CO}$ & Southwest & $X$ & $\mathrm{X}$ & $X$ & $\mathrm{X}$ & $\mathrm{X}$ & $\mathrm{X}$ & $X$ & $\mathrm{X}$ & $\mathrm{X}$ & $\mathrm{X}$ \\
\hline 09362000 & Lightner Creek near Durango, CO & Southwest & $\mathrm{X}$ & $\mathrm{X}$ & $\mathrm{X}$ & $\mathrm{X}$ & $\mathrm{X}$ & $\mathrm{X}$ & $\mathrm{X}$ & $\mathrm{X}$ & $\mathrm{X}$ & $\mathrm{X}$ \\
\hline 09363000 & Florida River near Durango, CO & Southwest & $\mathrm{X}$ & $\mathrm{X}$ & $\mathrm{X}$ & $\mathrm{X}$ & $X$ & $\mathrm{X}$ & -- & $X$ & $\mathrm{X}$ & $X$ \\
\hline 09363100 & Salt Creek near Oxford, CO & Southwest & $\mathrm{X}$ & $\mathrm{X}$ & $\mathrm{X}$ & $\mathrm{X}$ & $\mathrm{X}$ & -- & -- & $\mathrm{X}$ & $\mathrm{X}$ & $\mathrm{X}$ \\
\hline 09365500 & La Plata River at Hesperus, $\mathrm{CO}$ & Southwest & $\mathrm{X}$ & $\mathrm{X}$ & $X$ & $\mathrm{X}$ & $\mathrm{X}$ & $\mathrm{X}$ & $\mathrm{X}$ & $\mathrm{X}$ & $\mathrm{X}$ & $\mathrm{X}$ \\
\hline 09366000 & Cherry Creek near Red Mesa, CO & Southwest & $\mathrm{X}$ & $\mathrm{X}$ & $\mathrm{X}$ & $\mathrm{X}$ & -- & -- & -- & $X$ & $\mathrm{X}$ & $\mathrm{X}$ \\
\hline 09366500 & La Plata River at CO-NM State Line & Southwest & $\mathrm{X}$ & $\mathrm{X}$ & $\mathrm{X}$ & $\mathrm{X}$ & $\mathrm{X}$ & -- & -- & $\mathrm{X}$ & $\mathrm{X}$ & $\mathrm{X}$ \\
\hline 09367400 & La Plata River Tributary near Farmington, NM & Southwest & $\mathrm{X}$ & -- & -- & -- & -- & -- & -- & -- & $\mathrm{X}$ & $\mathrm{X}$ \\
\hline 09367530 & Locke Arroyo near Kirtland, NM & Southwest & $\mathrm{X}$ & -- & -- & -- & -- & -- & -- & -- & -- & -- \\
\hline 09367550 & Stevens Arroyo near Kirtland, NM & Southwest & $\mathrm{X}$ & -- & -- & -- & -- & -- & -- & -- & -- & -- \\
\hline 09367561 & Shumway Arroyo near Waterflow, NM & Southwest & $\mathrm{X}$ & $\mathrm{X}$ & $\mathrm{X}$ & $\mathrm{X}$ & -- & -- & -- & $\mathrm{X}$ & $\mathrm{X}$ & $\mathrm{X}$ \\
\hline 09367950 & Chaco River near Waterflow , NM & Southwest & $\mathrm{X}$ & $\mathrm{X}$ & $\mathrm{X}$ & $\mathrm{X}$ & -- & -- & -- & $\mathrm{X}$ & $\mathrm{X}$ & $\mathrm{X}$ \\
\hline 09367980 & Rattlesnake Arroyo near Shiprock, NM & Southwest & $\mathrm{X}$ & -- & -- & -- & -- & -- & -- & -- & -- & -- \\
\hline 09368020 & Malpais Arroyo near Shiprock, NM & Southwest & $\mathrm{X}$ & -- & -- & -- & -- & -- & -- & -- & -- & -- \\
\hline 09368500 & West Mancos River near Mancos, CO & Southwest & $\mathrm{X}$ & $\mathrm{X}$ & $\mathrm{X}$ & $\mathrm{X}$ & $\mathrm{X}$ & $\mathrm{X}$ & $\mathrm{X}$ & $\mathrm{X}$ & $\mathrm{X}$ & $\mathrm{X}$ \\
\hline 09369000 & East Mancos River near Mancos, CO & Southwest & $\mathrm{X}$ & $\mathrm{X}$ & $\mathrm{X}$ & $\mathrm{X}$ & $\mathrm{X}$ & $\mathrm{X}$ & -- & -- & -- & -- \\
\hline 09369500 & Middle Mancos River near Mancos, CO & Southwest & $\mathrm{X}$ & $\mathrm{X}$ & $\mathrm{X}$ & $\mathrm{X}$ & $\mathrm{X}$ & -- & -- & $\mathrm{X}$ & $\mathrm{X}$ & $\mathrm{X}$ \\
\hline 09371000 & Mancos River near Towaoc, $\mathrm{CO}$ & Southwest & $\mathrm{X}$ & $\mathrm{X}$ & $\mathrm{X}$ & $\mathrm{X}$ & -- & -- & -- & $\mathrm{X}$ & $\mathrm{X}$ & $\mathrm{X}$ \\
\hline 09371300 & Mcelmo Creek Tributary near Cortez, CO & Southwest & $\mathrm{X}$ & -- & -- & -- & -- & -- & -- & -- & -- & -- \\
\hline 09372000 & Mcelmo Creek near CO-UT State Line & Southwest & $\mathrm{X}$ & $\mathrm{X}$ & $\mathrm{X}$ & $\mathrm{X}$ & $\mathrm{X}$ & $\mathrm{X}$ & $\mathrm{X}$ & $\mathrm{X}$ & $\mathrm{X}$ & $\mathrm{X}$ \\
\hline 09378170 & South Creek Above Reservoir near Monticello, UT & Southwest & $\mathrm{X}$ & $\mathrm{X}$ & $\mathrm{X}$ & $\mathrm{X}$ & $\mathrm{X}$ & -- & -- & $\mathrm{X}$ & $\mathrm{X}$ & $\mathrm{X}$ \\
\hline 09378630 & Recapture Creek near Blanding, UT & Southwest & $\mathrm{X}$ & $\mathrm{X}$ & $\mathrm{X}$ & $\mathrm{X}$ & -- & -- & -- & & $\mathrm{X}$ & $\mathrm{X}$ \\
\hline 09378650 & Recapture Creek below Johnson Creek near Blanding, UT & Southwest & $\mathrm{X}$ & $\mathrm{X}$ & $\mathrm{X}$ & $\mathrm{X}$ & -- & -- & -- & $\mathrm{X}$ & $\mathrm{X}$ & $\mathrm{X}$ \\
\hline 09378700 & Cottonwood Wash near Blanding, UT & Southwest & $\mathrm{X}$ & $\mathrm{X}$ & $\mathrm{X}$ & $\mathrm{X}$ & -- & -- & -- & $\mathrm{X}$ & $\mathrm{X}$ & $\mathrm{X}$ \\
\hline 09379000 & Cob Wash near Bluff, UT & Southwest & $\mathrm{X}$ & $\mathrm{X}$ & $\mathrm{X}$ & $\mathrm{X}$ & -- & -- & -- & $\mathrm{X}$ & $\mathrm{X}$ & $\mathrm{X}$ \\
\hline 09379030 & Black Mountain Wash near Chinle, AZ & Southwest & $\mathrm{X}$ & -- & -- & -- & -- & -- & -- & -- & -- & $\mathrm{X}$ \\
\hline 09379060 & Lukachukai Creek Tributary near Lukachukai, AZ & Southwest & $\mathrm{X}$ & -- & -- & -- & -- & -- & -- & -- & -- & $\mathrm{X}$ \\
\hline 09379300 & Lime Creek near Mexican Hat, UT & Southwest & $\mathrm{X}$ & -- & -- & -- & -- & -- & -- & -- & -- & $\mathrm{X}$ \\
\hline
\end{tabular}


Publishing support provided by:

Denver Publishing Service Center, Denver, Colorado Manuscript approved for publication, June 17, 2009 Edited by Mary Kidd

Graphics and layout by Joy Monson

For more information concerning this publication, contact: Director, USGS Colorado Water Science Center Box 25046, Mail Stop 415

Denver, C0 80225 (303) 236-4882

Or visit the Colorado Water Science Center Web site at: http://co.water.usgs.gov/

This publication is available online at: http://pubs.usgs.gov/sir/2009/5136/ 
